\title{
Palladacycle-phosphine catalyzed methylation of amines and ketones using methanol
}

Ramesh Mamidala, ${ }^{\dagger}$ Priyabrata Biswal, ${ }^{\dagger}$ M. Siva Subramani, Shaikh Samser and Krishnan Venkatasubbaiah*

School of Chemical Sciences, National Institute of Science Education and Research (NISER), Bhubaneswar, HBNI, Bhubaneswar-752050, Odisha (India). E-mail: krishv@niser.ac.in $\dagger$ Equally contributed

\section{Table of Contents}

Figure S1-S2. Molecular structure of palladacycle-4 \&

compound 23

Table S1. Crystal data and structure refinement

parameters

Scheme S1: Proposed reaction mechanism for N-

methylation of amines

Figure S3-18. NMR spectra for pyrazoles and

palladacycles

Figure 19-70. NMR Spectra for C-methylated and N-

S13-S38

methylated compounds 
X-ray analysis: Crystallographic data for palladacycle 4 and compound 23 and details of Xray diffraction experiments and crystal structure refinements are given in Table S1. Using Olex2, the structures was solved with the ShelXS structure solution program using Direct Methods and refined with the ShelXL refinement package using Least Squares minimization. All non-hydrogen atoms were refined with anisotropic displacement coefficients. The disordered fluorine atoms (F10-12) were treated and refined with two independent positions. The $\mathrm{H}$ atoms were placed at calculated positions were refined as riding atoms. Crystallographic data for the structure $\mathbf{4}$ and compound $\mathbf{2 3}$ has been deposited with the Cambridge Crystallographic Data Center as supplementary publication no. CCDC-1906689-1906690. Copies of the data can be obtained free of charge on application to CCDC, 12 Union Road, Cambridge CB2 1EZ, UK (fax: (+44) 1223-336-033; email: deposit@ccdc.cam.ac.uk

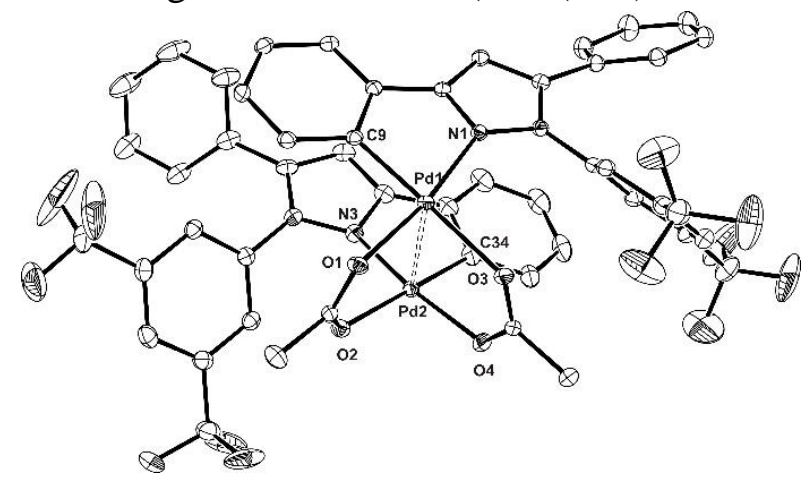

Figure S1: Molecular structure of palladacycle 4 (30\% probability thermal ellipsoids). Hydrogen atoms are omitted for clarity. Selected bond lengths $(\AA)$ and bond angles (degree): Pd1-C9 1.971(3), Pd1-N1 2.034(2), Pd1-O1 2.034(2), Pd1-O3 2.138(2), Pd1-Pd2 2.8731(3), C9-Pd1-N1 80.34(11), C9-Pd1-O1 91.11(11), N1-Pd1-O1 171.30(9), C9-Pd1-O3 178.99(10), N1-Pd1-O3 100.53(9), O3-Pd1-O1 88.04(9), C9-Pd1-Pd2 100.83(8), N1-Pd1-Pd2 97.30(7), O1-Pd1-Pd2 85.82(6), O3-Pd1-Pd2 78.56(6), Pd2-C34 1.965(3), Pd2-N3 2.035(3), Pd2-O2 2.142(2), Pd2-O4 2.021(2).

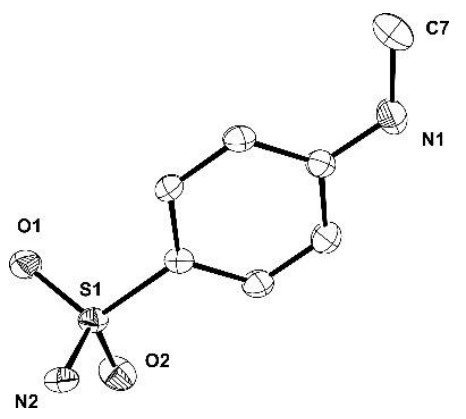

Figure S2: Molecular structure of compound 23 (30\% probability thermal ellipsoids). 
Table S1: Crystal data and structure refinement parameters for the palladacycle-4 \& compound-23.

\begin{tabular}{|c|c|c|}
\hline Identification code & Palladacycle-4 & Compound 23 \\
\hline Empirical formula & $\begin{array}{l}\mathrm{C}_{53} \mathrm{H}_{37} \mathrm{Cl}_{6} \\
\mathrm{~F}_{12} \mathrm{~N}_{4} \mathrm{O}_{4} \mathrm{Pd}_{2}\end{array}$ & $\mathrm{C}_{7} \mathrm{H}_{10} \mathrm{~N}_{2} \mathrm{O}_{2} \mathrm{~S}$ \\
\hline Formula weight & 1447.36 & 186.23 \\
\hline Temperature/K & 296.15 & 293(2) \\
\hline Crystal system & triclinic & orthorhombic \\
\hline Space group & $\mathrm{P}-1$ & $\mathrm{Pna}_{1}$ \\
\hline $\mathrm{a} / \AA$ & $12.6732(14)$ & $17.0270(4)$ \\
\hline $\mathrm{b} / \AA$ & $15.4639(7)$ & $7.43120(16)$ \\
\hline $\mathrm{c} / \AA$ & $16.0509(8)$ & $6.87490(14)$ \\
\hline$\alpha /^{\circ}$ & $114.873(2)$ & 90 \\
\hline$\beta /{ }^{\circ}$ & $96.893(3)$ & 90 \\
\hline$\gamma /{ }^{\circ}$ & $94.600(3)$ & 90 \\
\hline Volume $/ \AA^{3}$ & $2803.2(4)$ & $869.89(3)$ \\
\hline $\mathrm{Z}$ & 2 & 4 \\
\hline$\rho_{\text {calc }} \mathrm{g} / \mathrm{cm}^{3}$ & 1.715 & 1.422 \\
\hline$\mu / \mathrm{mm}^{-1}$ & 1.016 & 3.016 \\
\hline $\mathrm{F}(000)$ & 1434.0 & 392.0 \\
\hline Radiation & $\begin{array}{l}\operatorname{MoK} \alpha \quad(\lambda \\
0.71073)\end{array}$ & $=\mathrm{CuK} \alpha(\lambda=1.54184)$ \\
\hline$\theta$ range $\left[{ }^{\circ}\right]$ & 3.95 to 56.77 & 10.39 to 133.184 \\
\hline Index ranges & $\begin{array}{l}-16 \leq \mathrm{h} \leq 16 \\
-20 \leq \mathrm{k} \leq 20 \\
-21 \leq 1 \leq 19\end{array}$ & $\begin{array}{l}-20 \leq \mathrm{h} \leq 20 \\
-7 \leq \mathrm{k} \leq 8 \\
-8 \leq 1 \leq 7\end{array}$ \\
\hline Reflections collected & 45908 & 3649 \\
\hline Independent reflections & $\begin{array}{l}13761 \\
{\left[R_{\text {int }}=0.0369\right.} \\
\left.R_{\text {sigma }}=0.0373\right]\end{array}$ & $\begin{array}{l}1230 \\
{\left[\mathrm{R}_{\text {int }}=0.0420\right.} \\
\left.\mathrm{R}_{\text {sigma }}=0.0417\right]\end{array}$ \\
\hline $\begin{array}{l}\text { Data/restraints/paramete } \\
\text { rs }\end{array}$ & $13761 / 0 / 759$ & $1230 / 1 / 124$ \\
\hline Goodness-of-fit on $\mathrm{F}^{2}$ & 1.033 & 1.071 \\
\hline $\begin{array}{l}\text { Final } R \text { indexes }[\mathrm{I}>=2 \sigma \\
\text { (I)] }\end{array}$ & $\begin{array}{l}\mathrm{R}_{1}=0.0416 \\
\mathrm{wR}_{2}=0.1044\end{array}$ & $\begin{array}{l}\mathrm{R}_{1}=0.0318 \\
\mathrm{wR}_{2}=0.0783\end{array}$ \\
\hline Final R indexes [all data] & $\begin{array}{l}\mathrm{R}_{1}=0.0530 \\
\mathrm{wR}_{2}=0.1124\end{array}$ & $\begin{array}{l}\mathrm{R}_{1}=0.0348 \\
\mathrm{wR}_{2}=0.0799\end{array}$ \\
\hline $\begin{array}{l}\text { Largest diff. peak/hole / } \\
\mathrm{e} \AA^{-3}\end{array}$ & / $1.43 /-1.73$ & $0.17 /-0.20$ \\
\hline
\end{tabular}


Scheme S1: Proposed reaction mechanism for $\mathrm{N}$ - methylation of amines

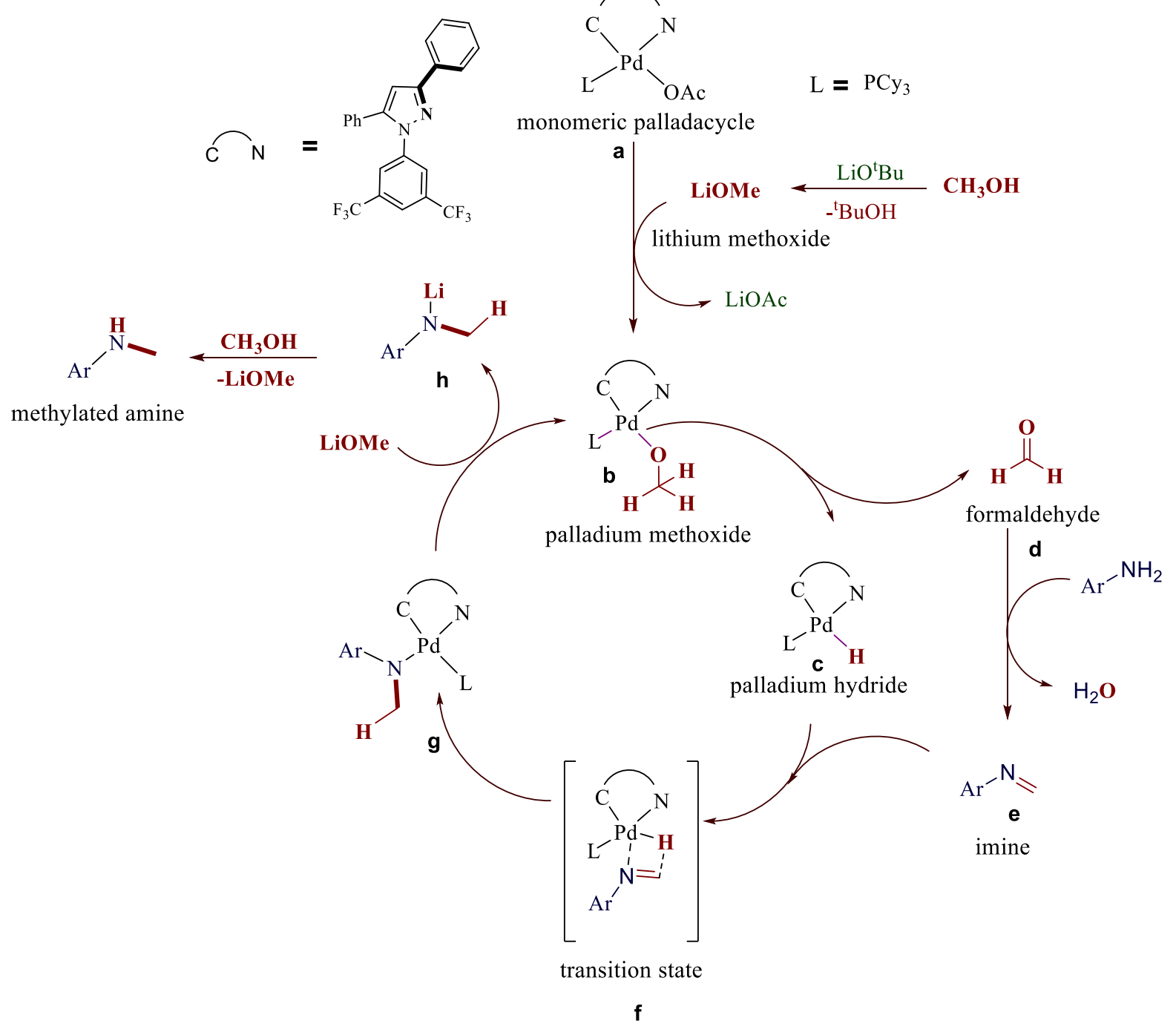



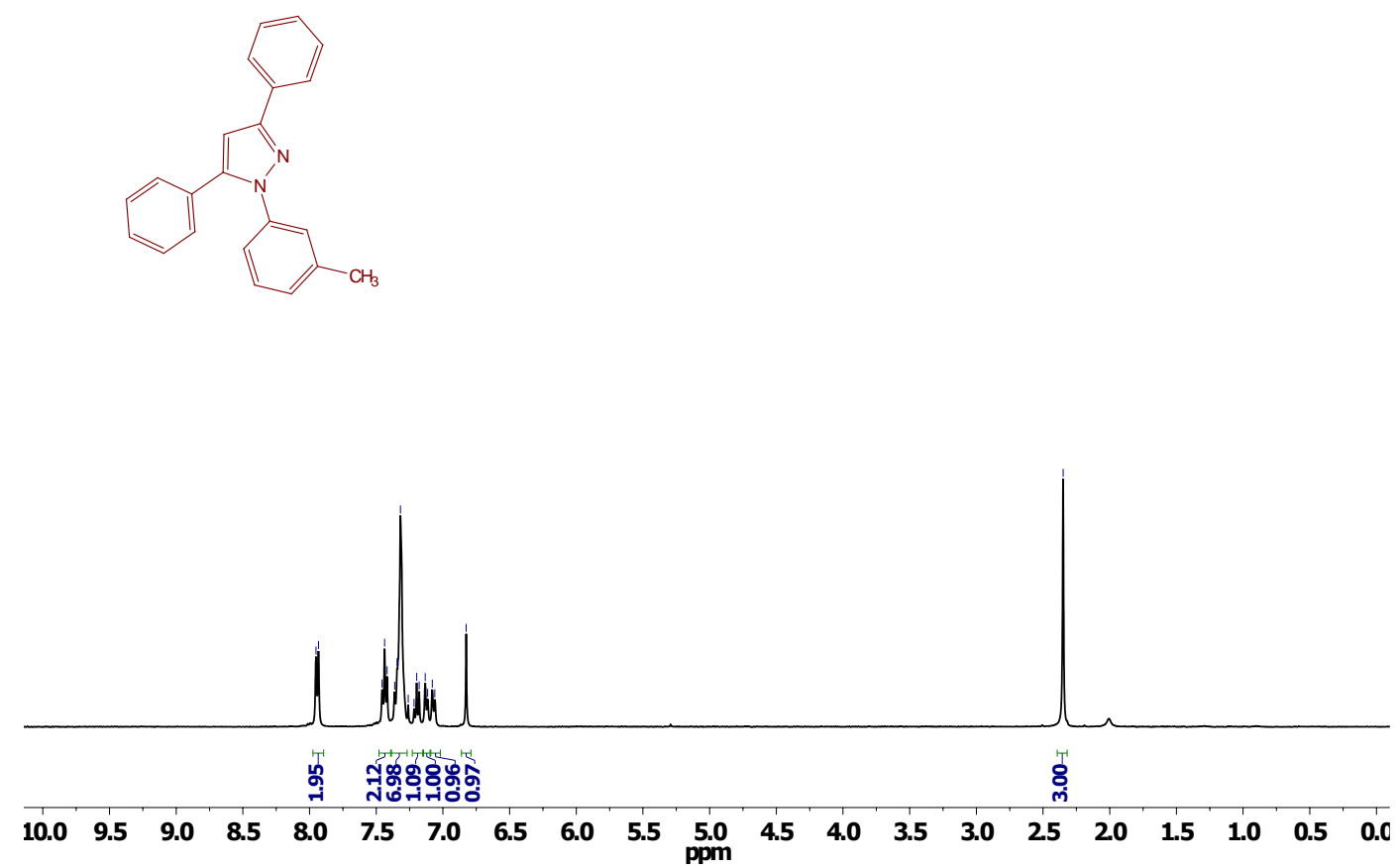

Figure S3. ${ }^{1} \mathrm{H}$ NMR of 3, 5-diphenyl-1-(m-tolyl)-1H-pyrazole

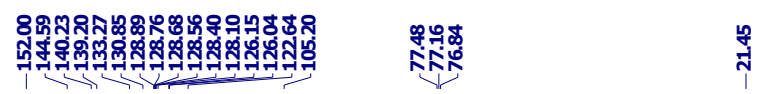
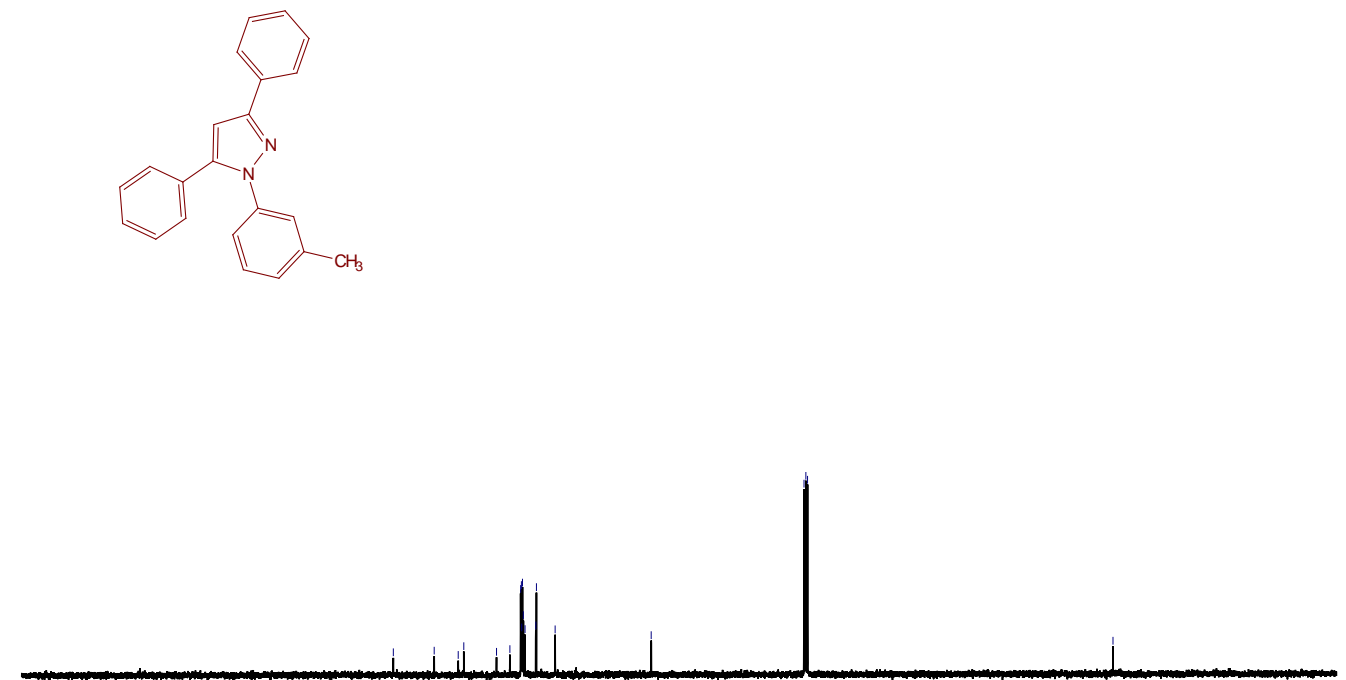

$\begin{array}{llllllllllllllllllllllll}210 & 200 & 190 & 180 & 170 & 160 & 150 & 140 & 130 & 120 & 110 & \begin{array}{c}100 \\ \mathrm{ppm}\end{array} & 90 & 80 & 70 & 60 & 50 & 40 & 30 & 20 & 10 & 0 & -10\end{array}$

Figure S4. ${ }^{13} \mathrm{C}$ NMR of 3, 5-diphenyl-1-(m-tolyl)-1H-pyrazole 

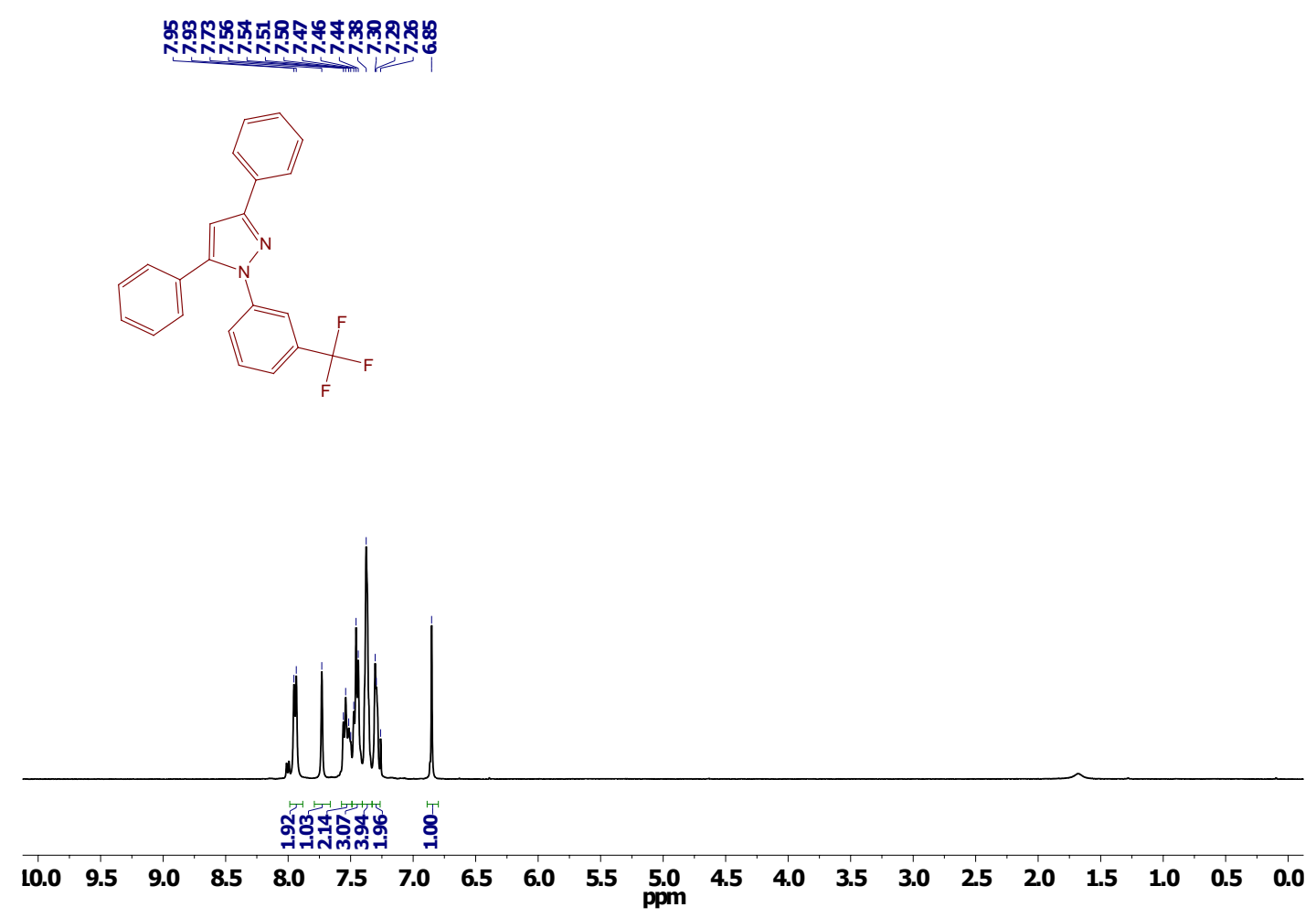

Figure S5. ${ }^{1} \mathrm{H}$ NMR of 3,5-Diphenyl-1-(3-(trifluoromethyl)phenyl)-1H-pyrazole
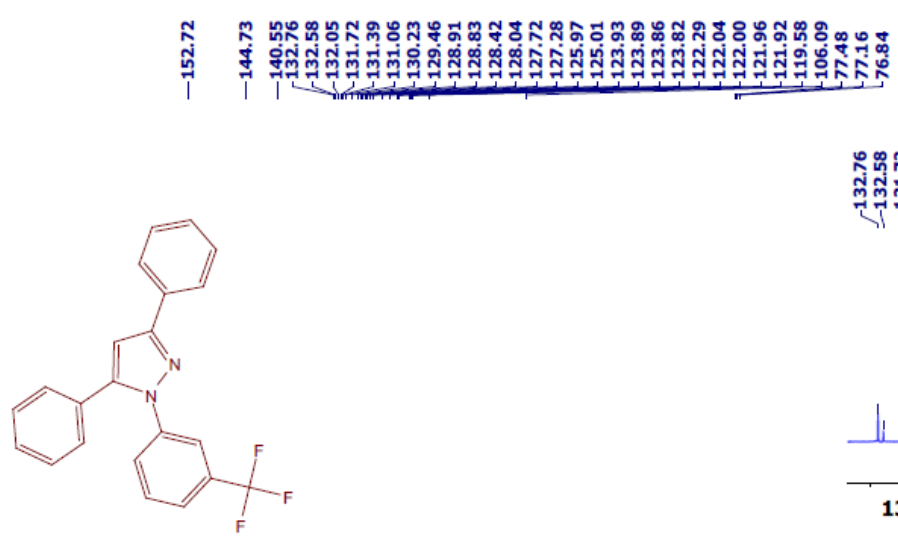

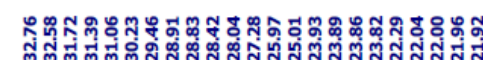
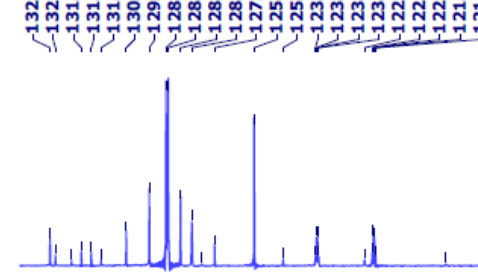

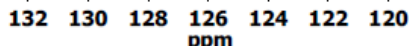

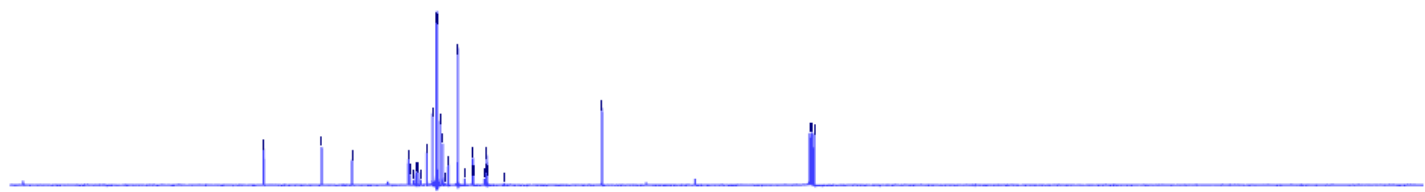

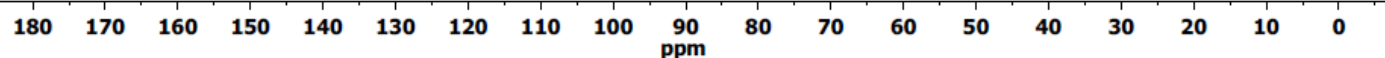

Figure S6. ${ }^{13} \mathrm{C}$ NMR of 3,5-Diphenyl-1-(3-(trifluoromethyl)phenyl)-1H-pyrazole 


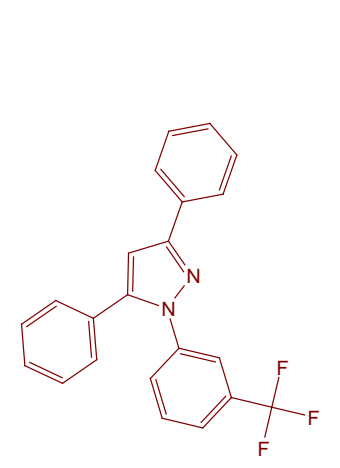

$\underset{\substack{0 \\ \mid}}{1}$

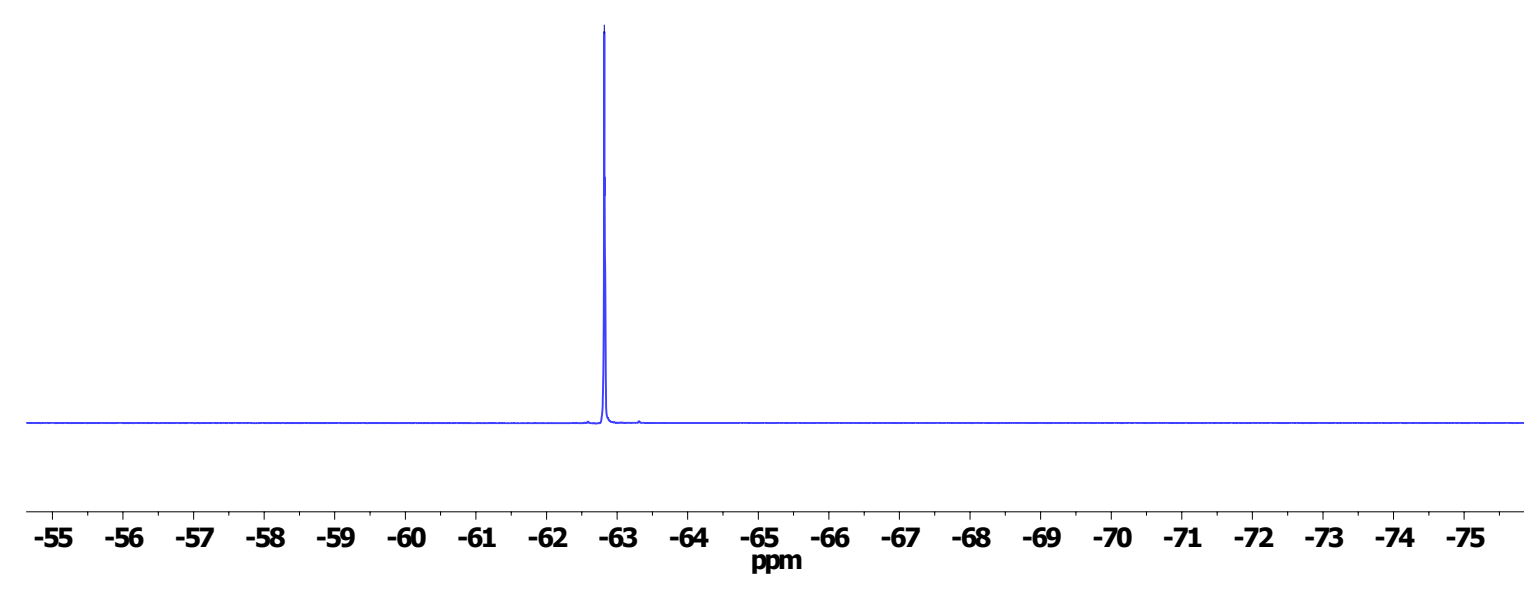

Figure S7. ${ }^{19}$ F NMR of 3,5-Diphenyl-1-(3-(trifluoromethyl)phenyl)-1H-pyrazole

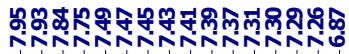
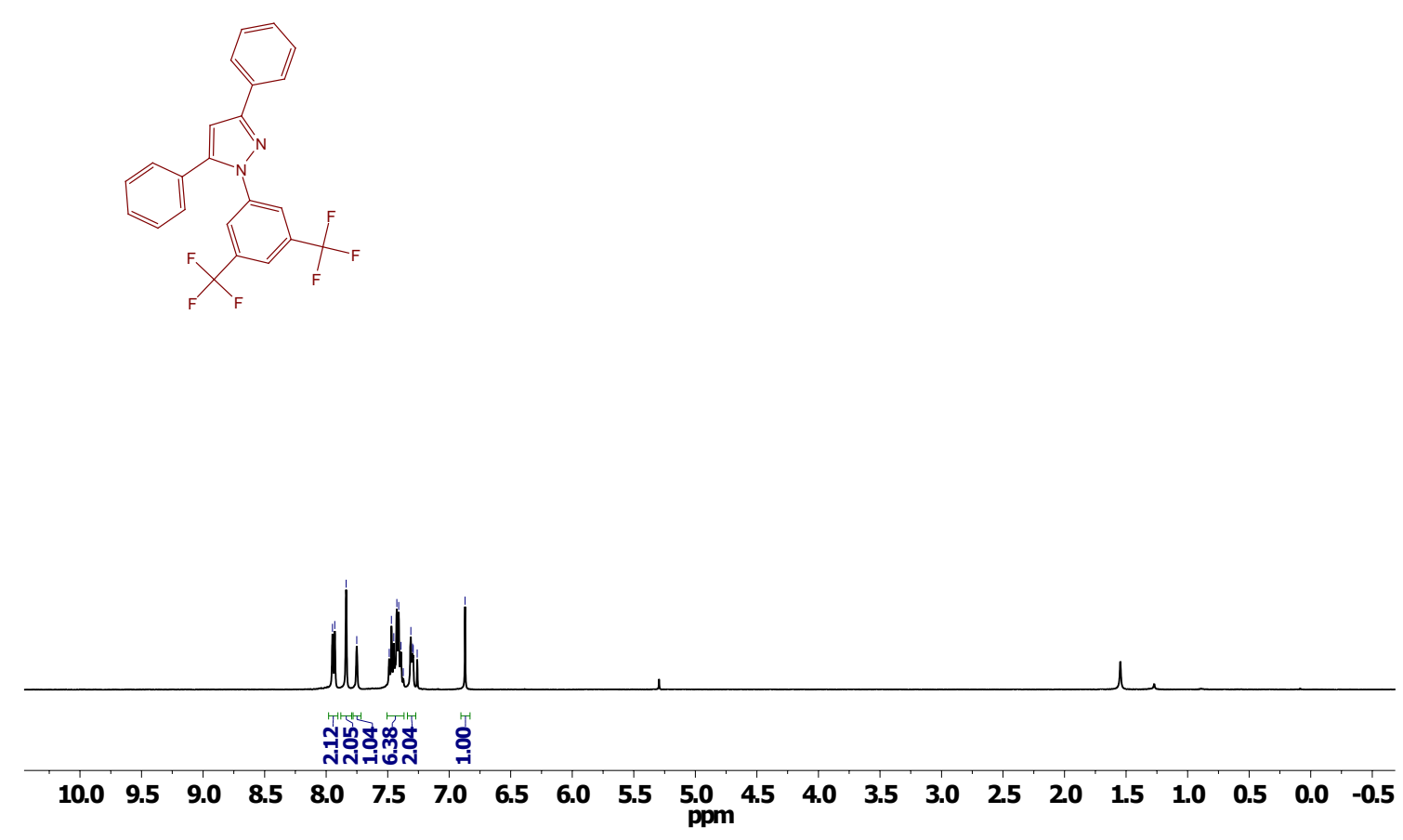

Figure S8. ${ }^{1} \mathrm{H}$ NMR of 1-(3,5-bis(trifluoromethyl)phenyl)-3,5-diphenyl-1H-pyrazole 


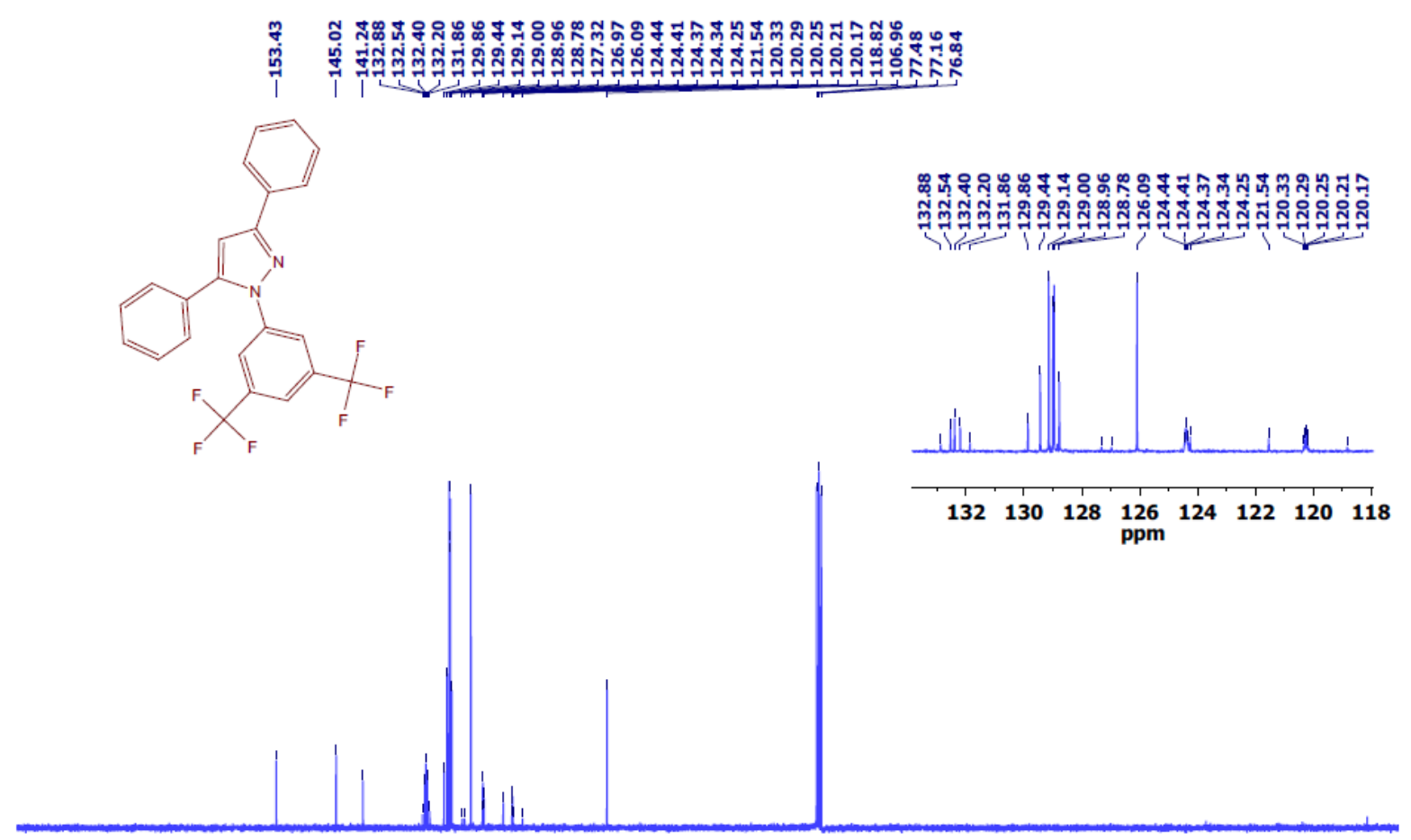

$\begin{array}{llllllllllllllllllll}180 & 170 & 160 & 150 & 140 & 130 & 120 & 110 & 100 & 90 & 80 & 70 & 60 & 50 & 40 & 30 & 20 & 10 & 0\end{array}$

Figure S9. ${ }^{13} \mathrm{C}$ NMR of 1-(3,5-bis(trifluoromethyl)phenyl)-3,5-diphenyl-1H-pyrazole

$\stackrel{\infty}{\not \oplus}$
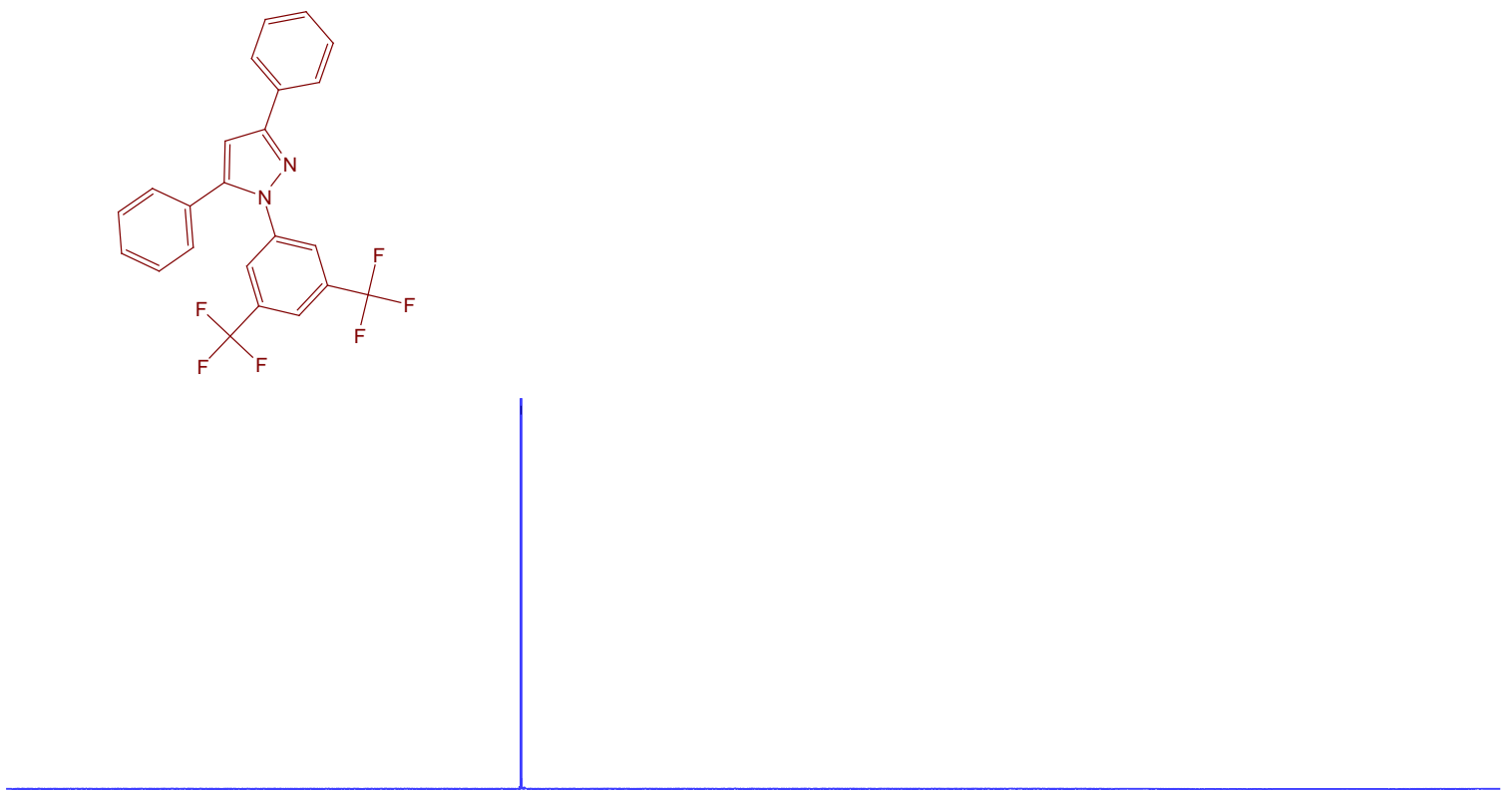

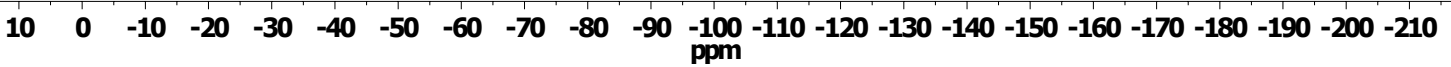

Figure S10. ${ }^{19}$ F NMR of 1-(3,5-bis(trifluoromethyl)phenyl)-3,5-diphenyl-1H-pyrazole 

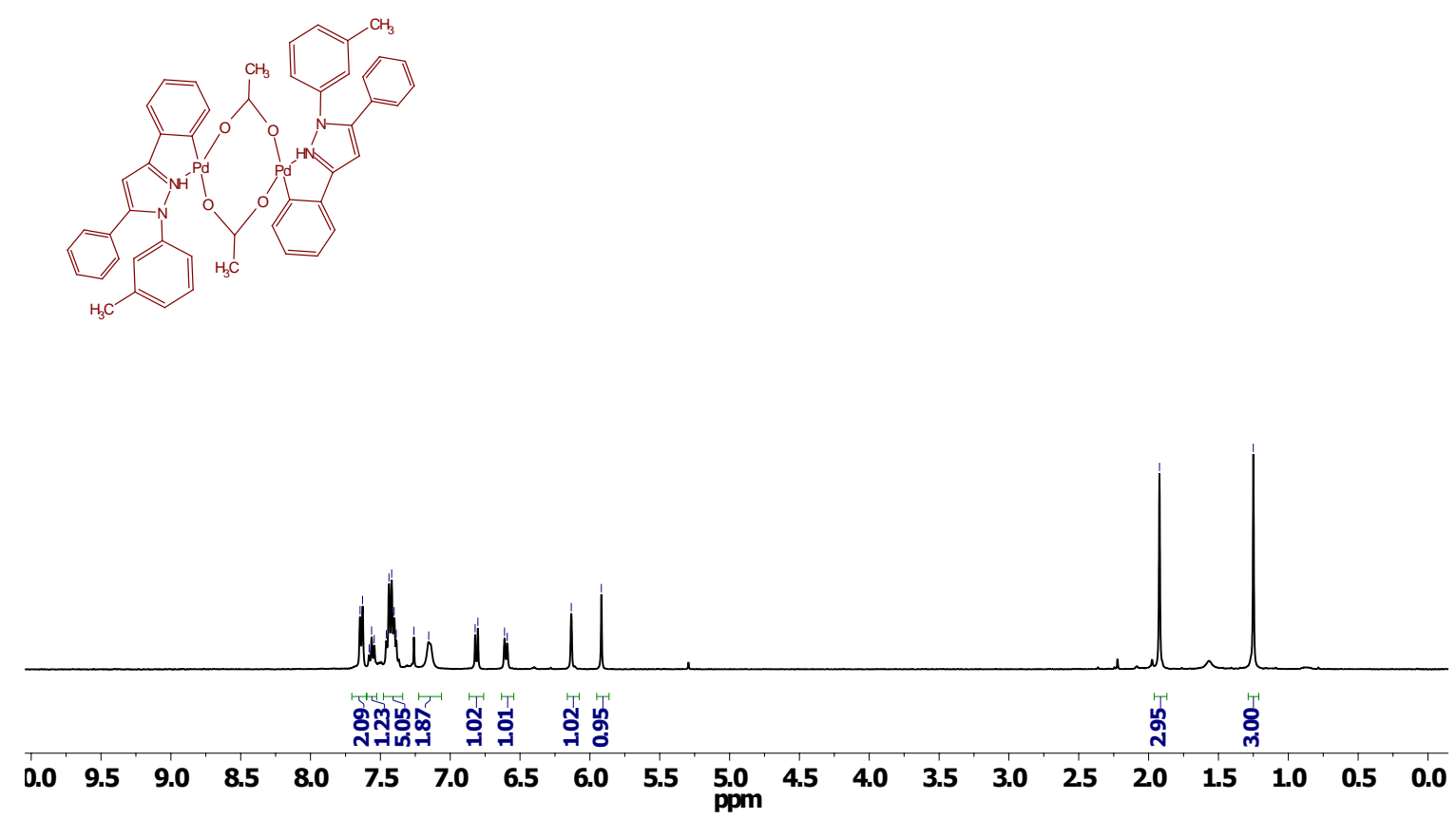

Figure S11. ${ }^{1} \mathrm{H}$ NMR of Palladacycle-2
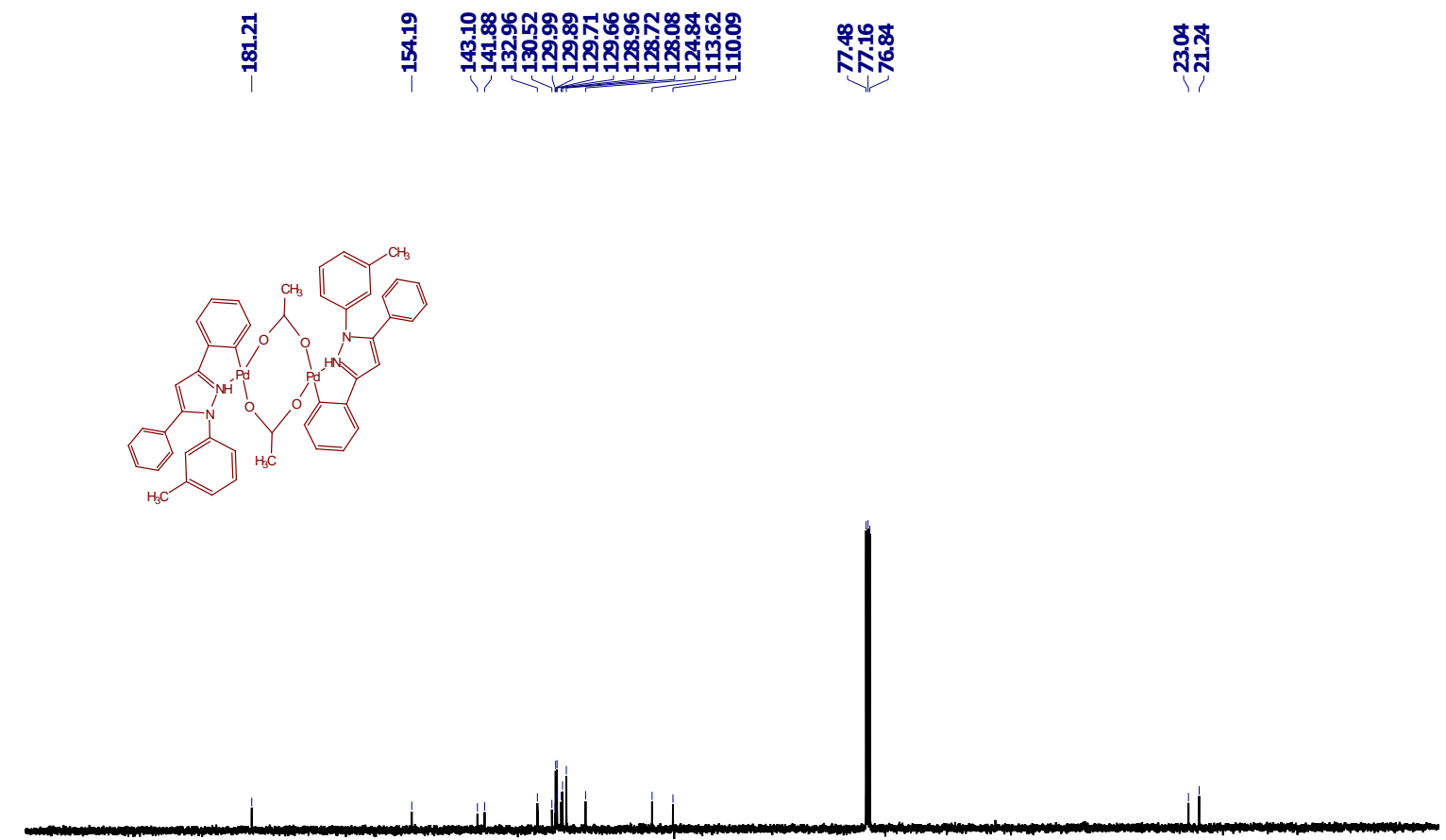

$\begin{array}{llllllllllllllllllllllllllll}210 & 200 & 190 & 180 & 170 & 160 & 150 & 140 & 130 & 120 & 110 & \mathbf{1 0 0} & 90 & 80 & 70 & 60 & 50 & 40 & 30 & 20 & 10 & 0 & -10\end{array}$

Figure S12. ${ }^{13} \mathrm{C}$ NMR of Palladacycle-2 

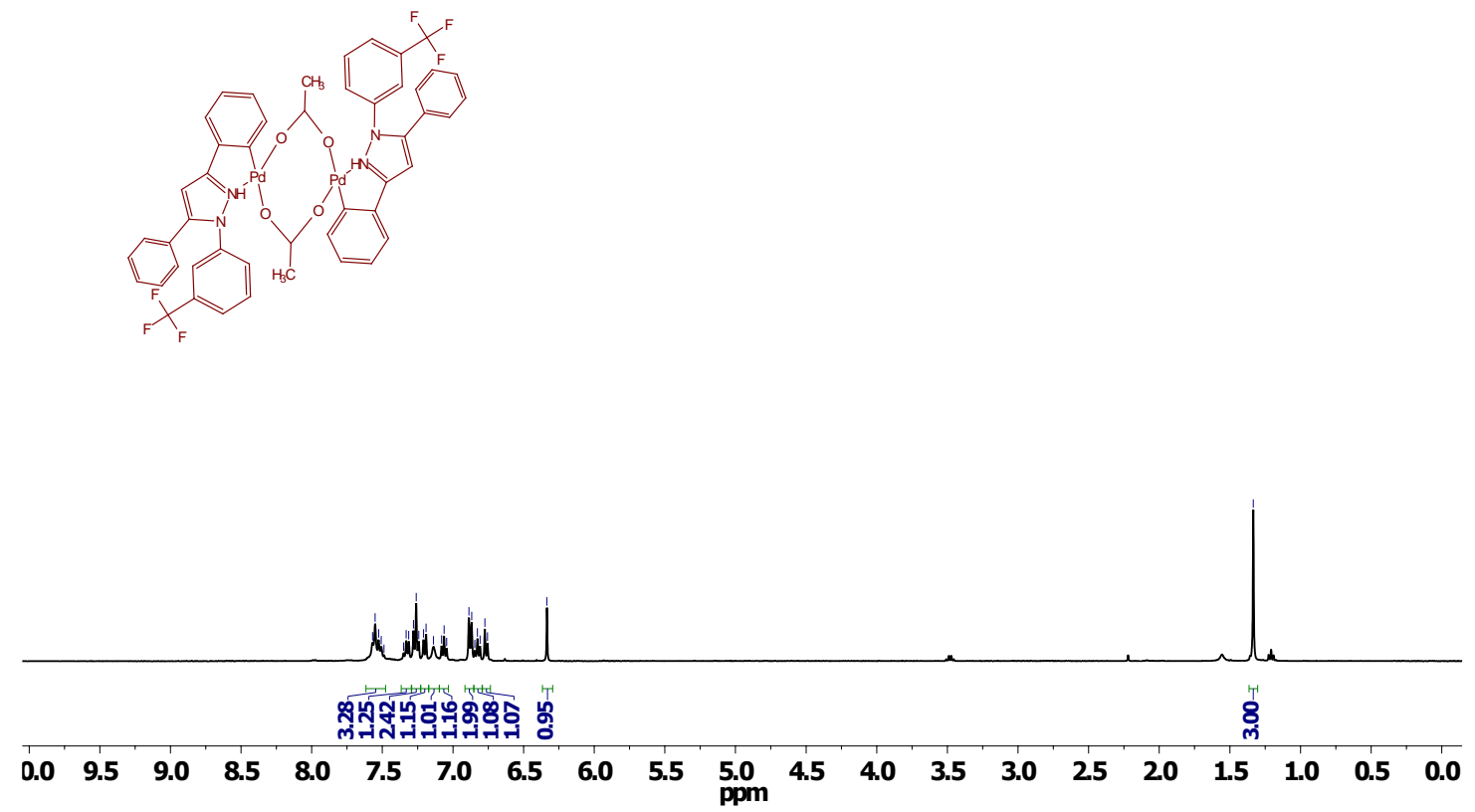

Figure S13. ${ }^{1} \mathrm{H}$ NMR of Palladacycle-3

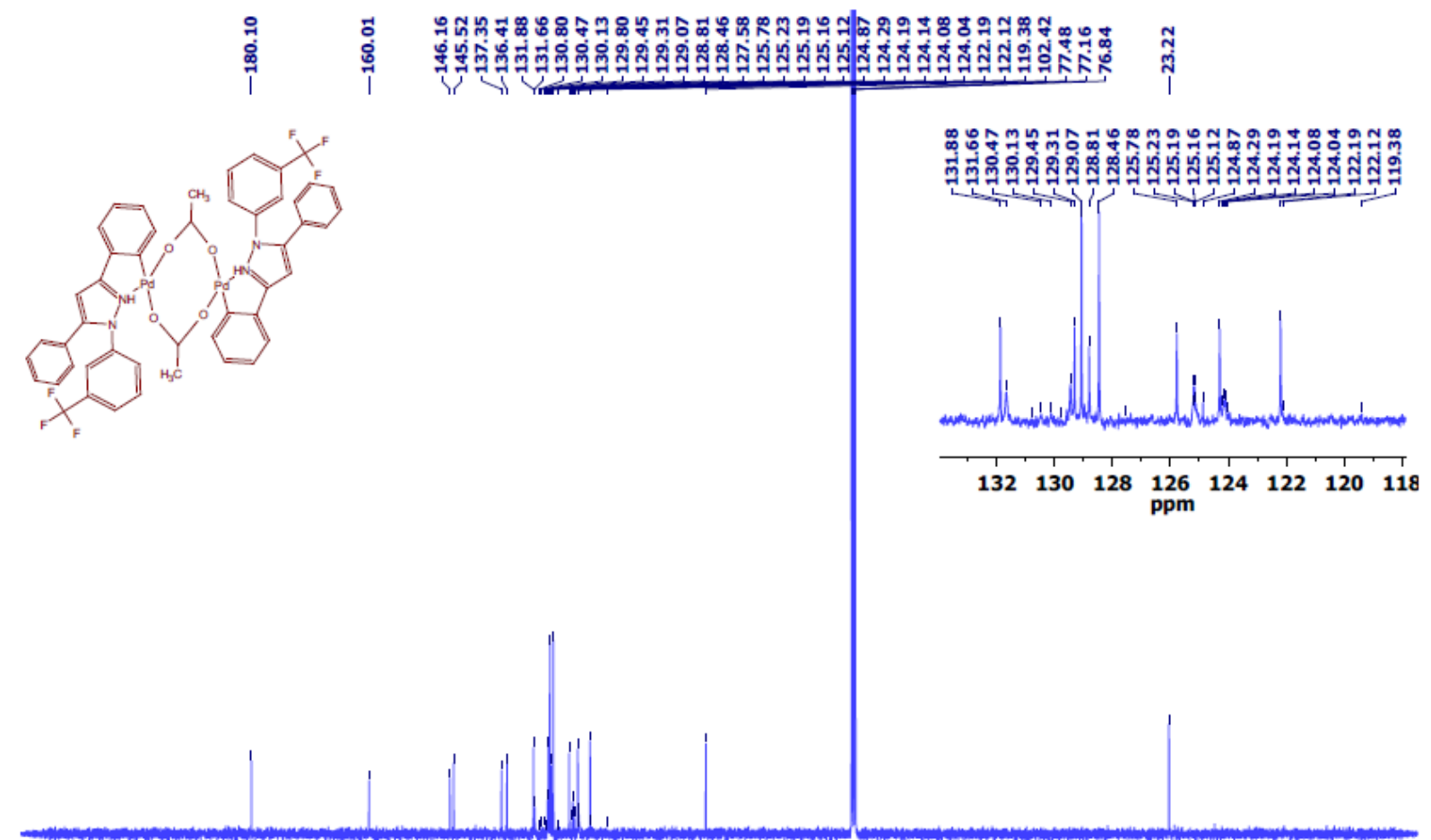

$\begin{array}{llllllllllllllllllllllll}210 & 200 & 190 & 180 & 170 & 160 & 150 & 140 & 130 & 120 & 110 & 100 & 90 & 80 & 70 & 60 & 50 & 40 & 30 & 20 & 10 & 0 & -10\end{array}$

Figure S14. ${ }^{13} \mathrm{C}$ NMR of Palladacycle-3 


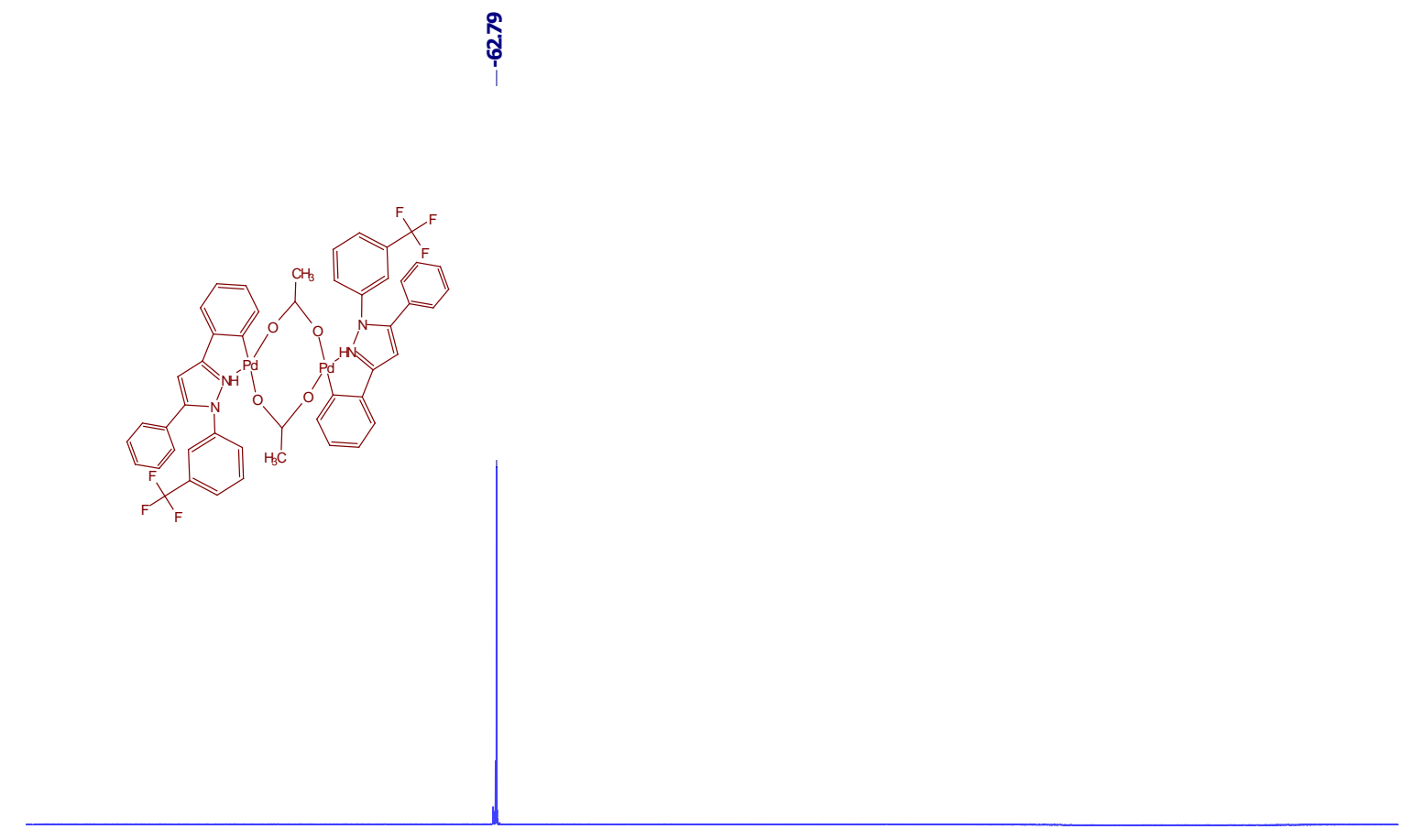

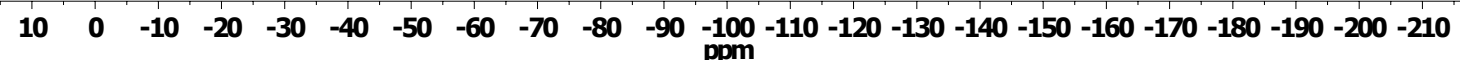

Figure S15. ${ }^{19} \mathrm{~F}$ NMR of Palladacycle-3

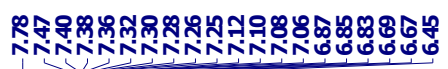
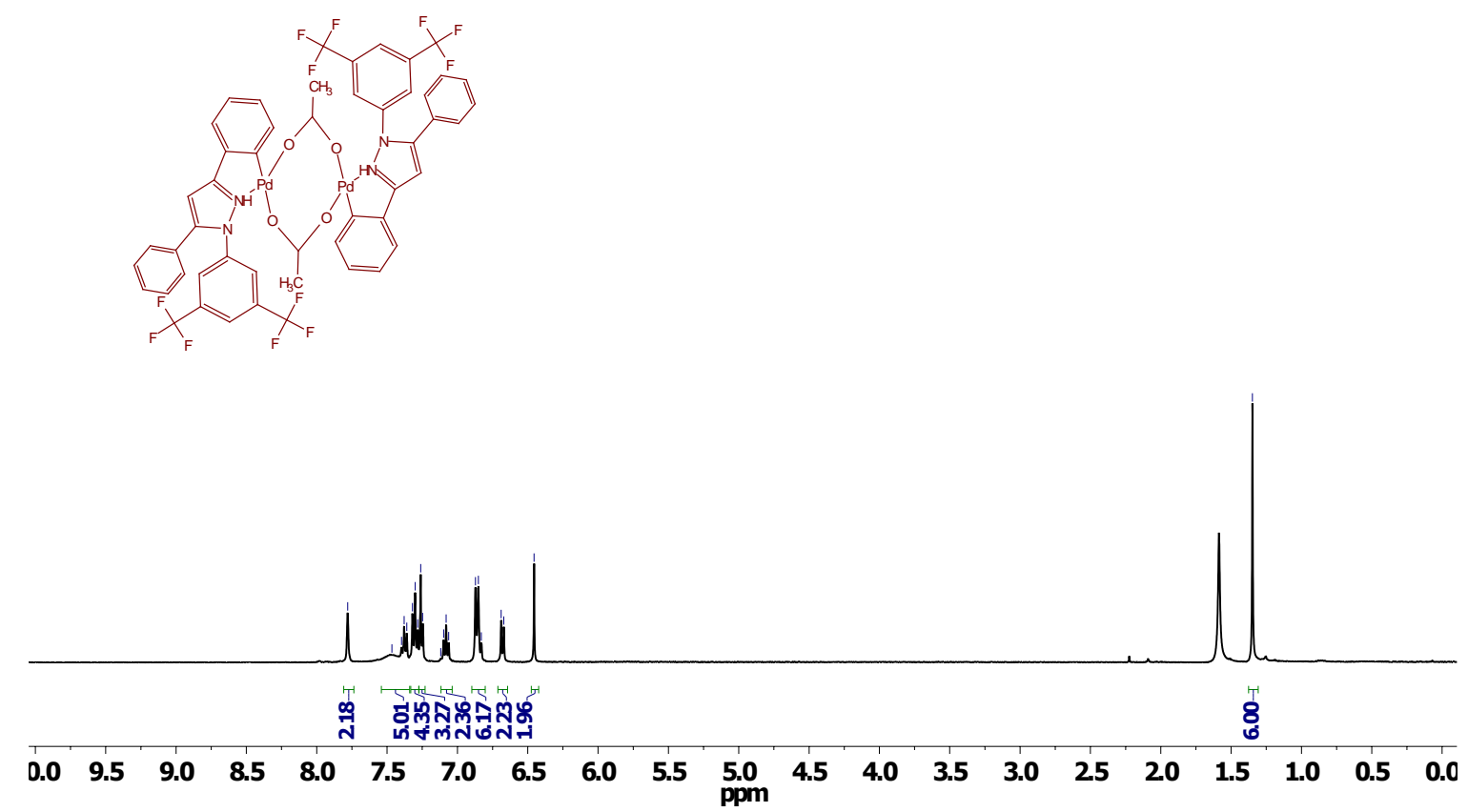

Figure S16. ${ }^{1} \mathrm{H}$ NMR of Palladacycle-4 

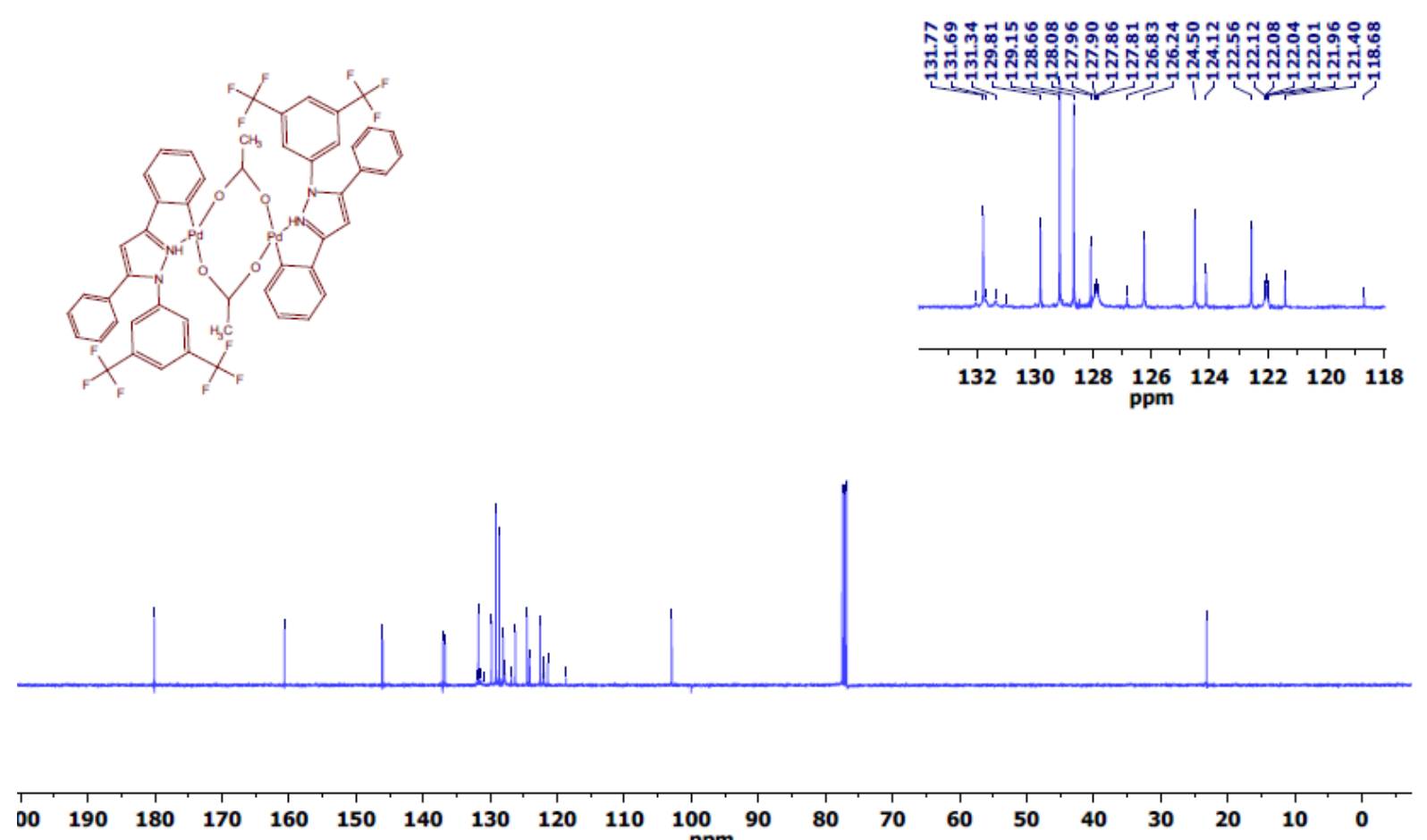

Figure S17. ${ }^{13} \mathrm{C}$ NMR of Palladacycle-4

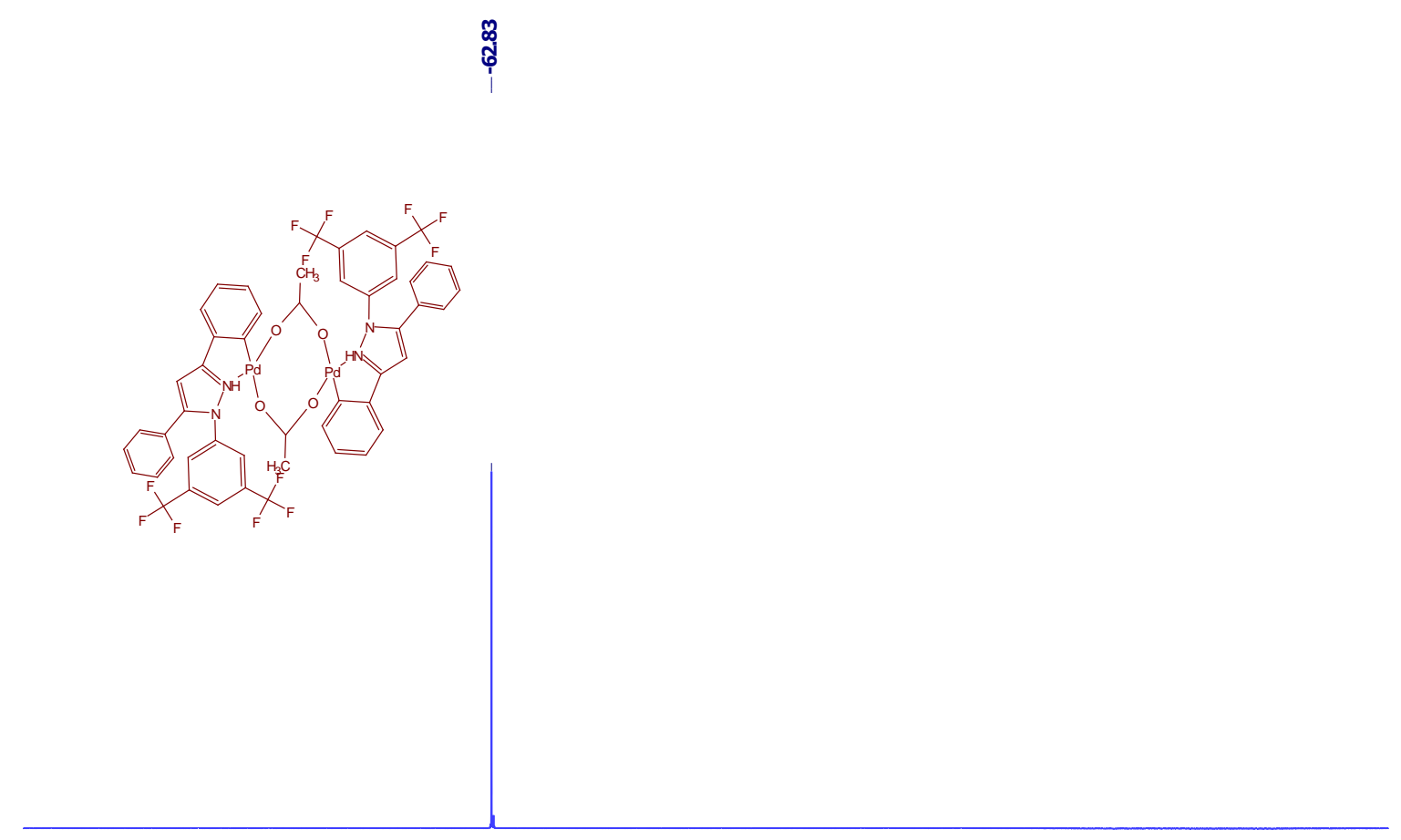

$10 \quad \begin{array}{llllllllllllllllllllllll}0 & -10 & -20 & -30 & -40 & -50 & -60 & -70 & -80 & -90 & -100 & -110 & -120 & -130 & -140 & -150 & -160 & -170 & -180 & -190 & -200 & -210\end{array}$

Figure S18. ${ }^{19} \mathrm{~F}$ NMR of Palladacycle-4 

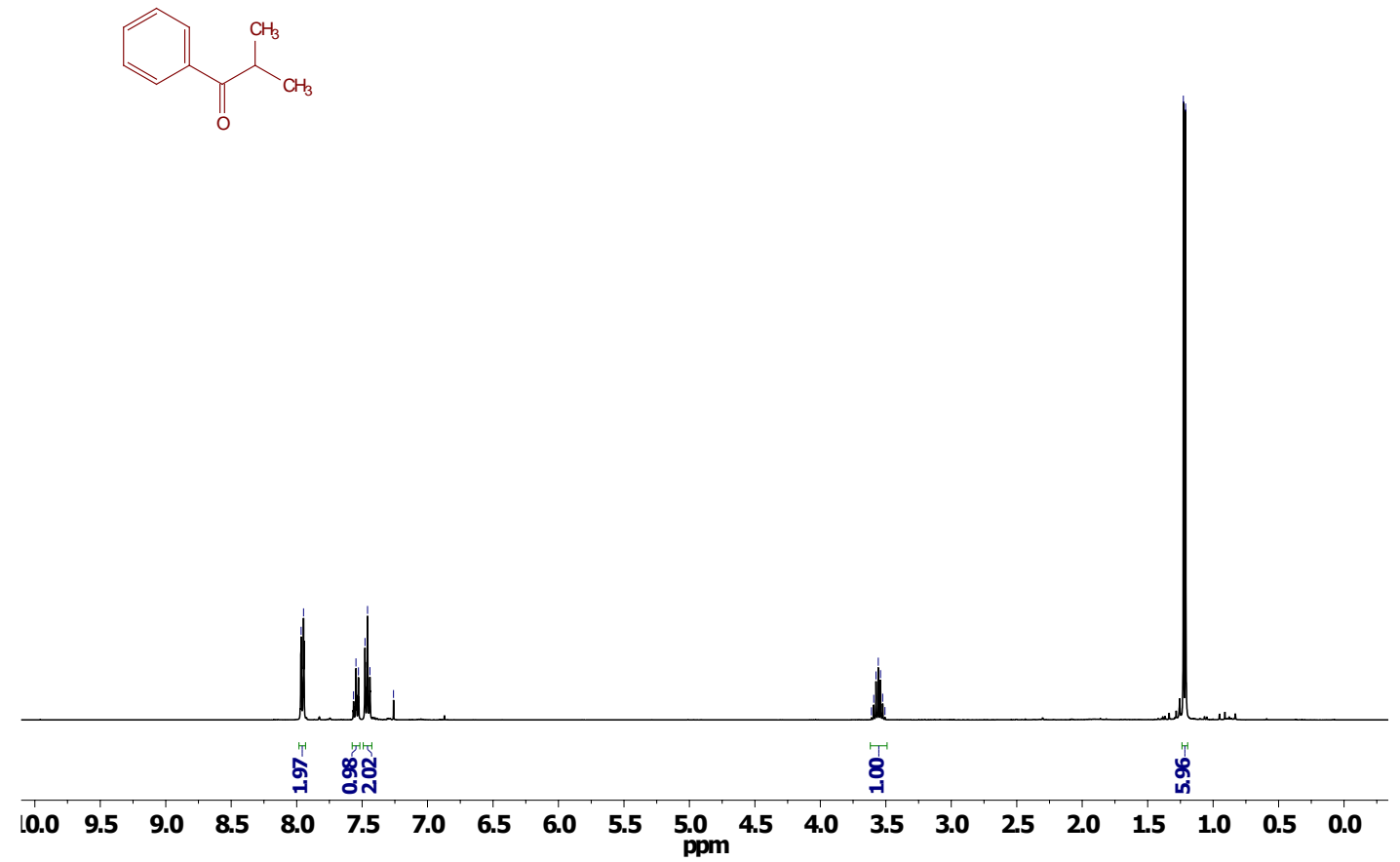

Figure S19. ${ }^{1} \mathrm{H}$ NMR of 2-methyl-1-phenylpropan-1-one

离
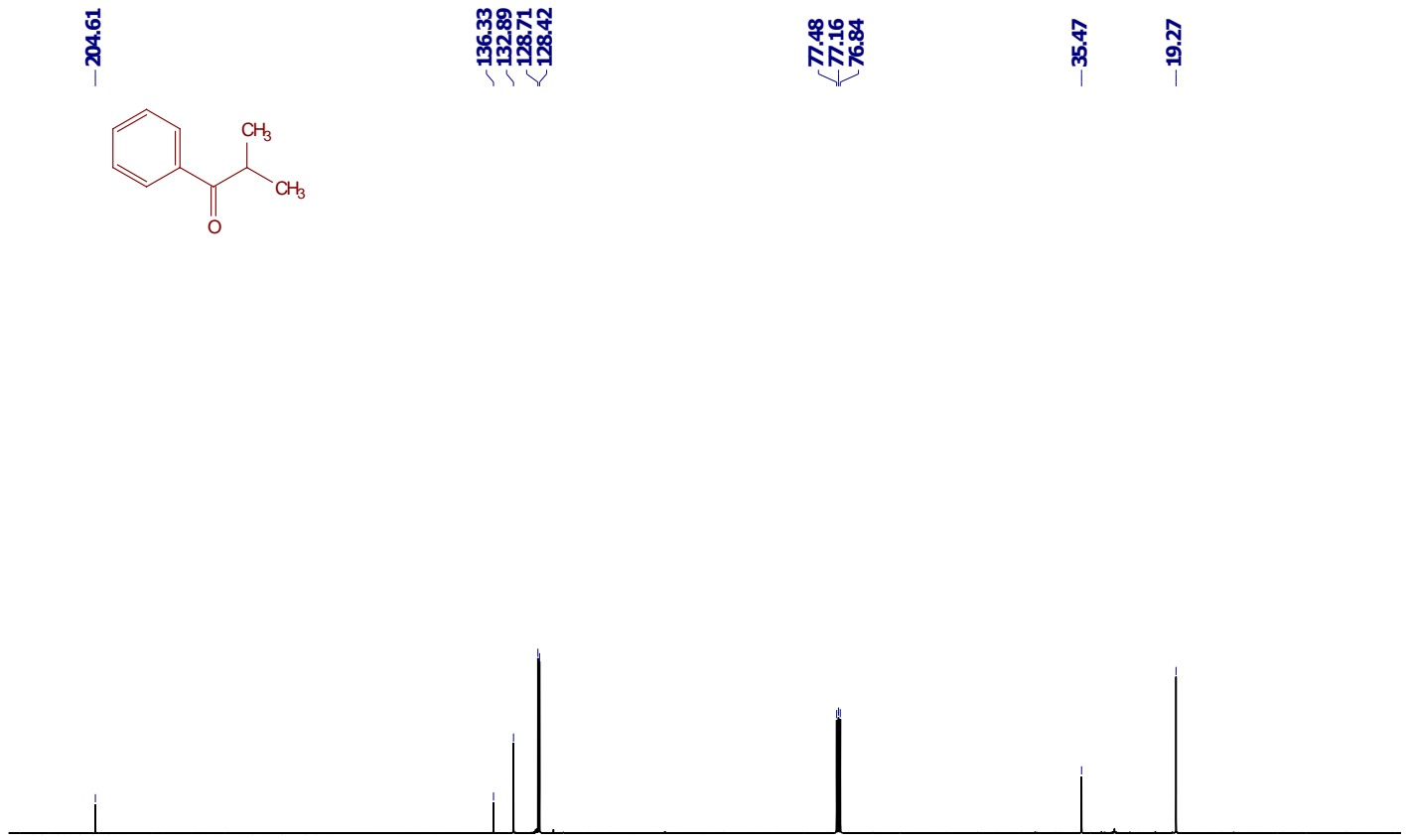

$\begin{array}{lllllllllllllllllllllll}210 & 200 & 190 & 180 & 170 & 160 & 150 & 140 & 130 & 120 & 110 & 100 & 90 & 80 & 70 & 60 & 50 & 40 & 30 & 20 & 10 & 0 & -10\end{array}$

Figure S20. ${ }^{13} \mathrm{C}$ NMR of 2-methyl-1-phenylpropan-1-one 


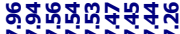

$\overbrace{0}^{C_{2}} \mathrm{CH}_{3}$

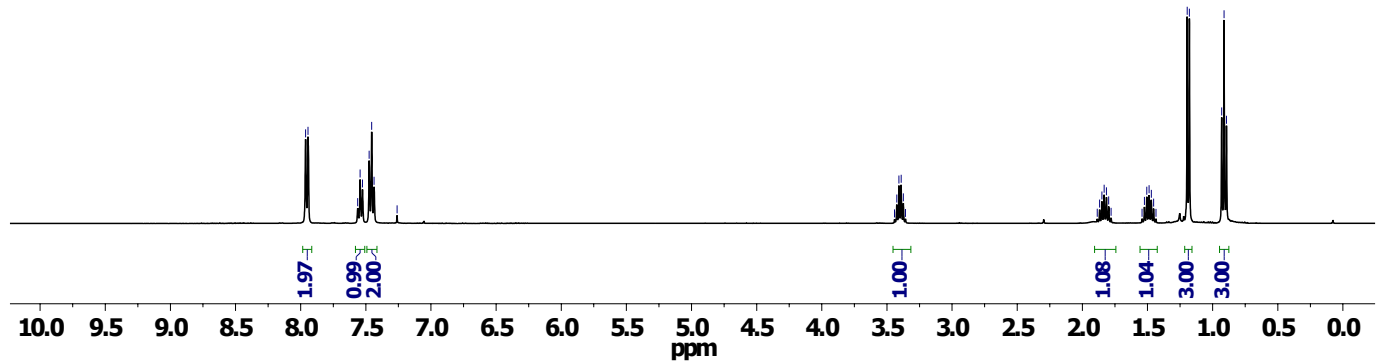

Figure S21. ${ }^{1} \mathrm{H}$ NMR 2-methyl-1-phenylbutan-1-one
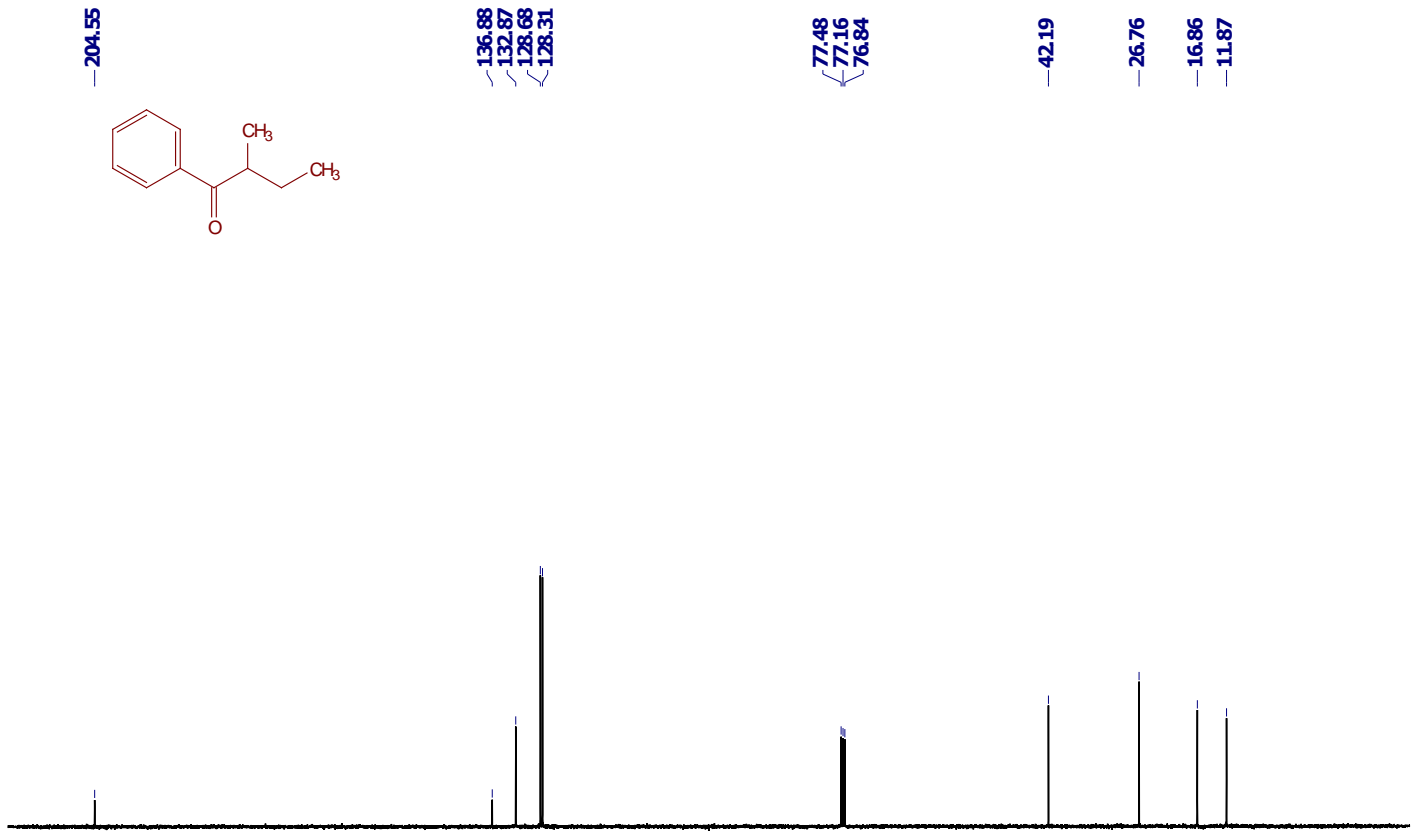

$\begin{array}{llllllllllllllllllllllllllllllllll}210 & 200 & 190 & 180 & 170 & 160 & 150 & 140 & 130 & 120 & 110 & 100 & 90 & 80 & 70 & 60 & 50 & 40 & 30 & 20 & 10 & 0 & -10\end{array}$

Figure S22. ${ }^{13} \mathrm{C}$ NMR of 2-methyl-1-phenylbutan-1-one 


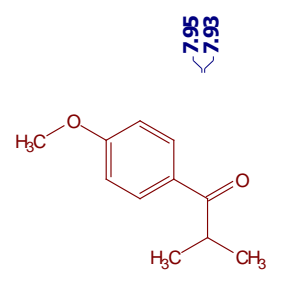

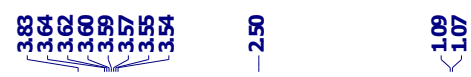

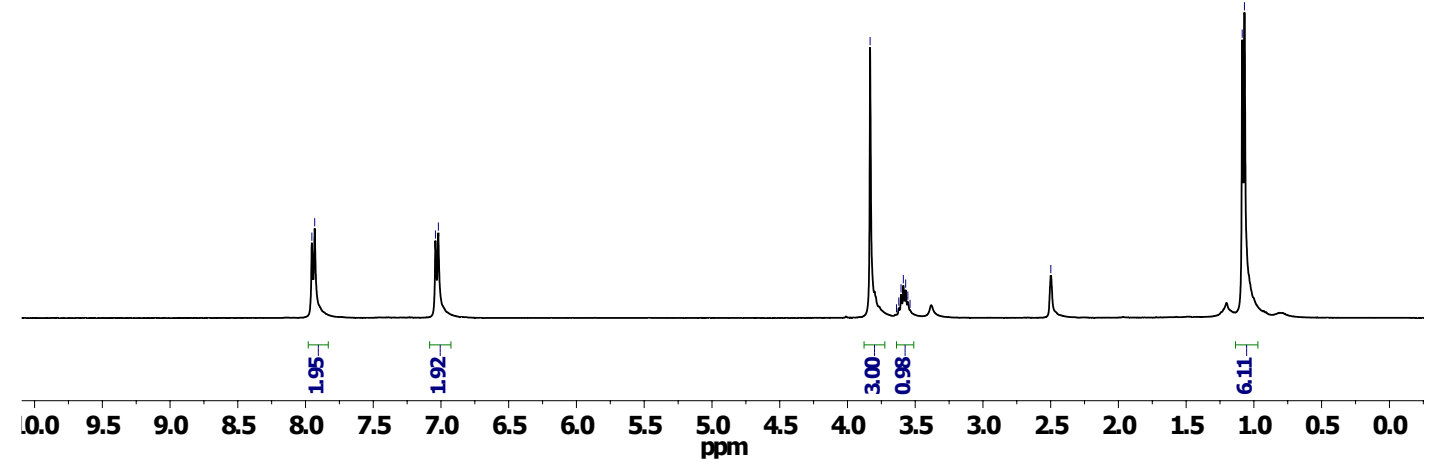

Figure S23. ${ }^{1} \mathrm{H}$ NMR 2-methyl-1-phenylbutan-1-one<smiles>COc1ccc(C(=O)C(C)C)cc1</smiles>

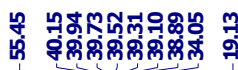

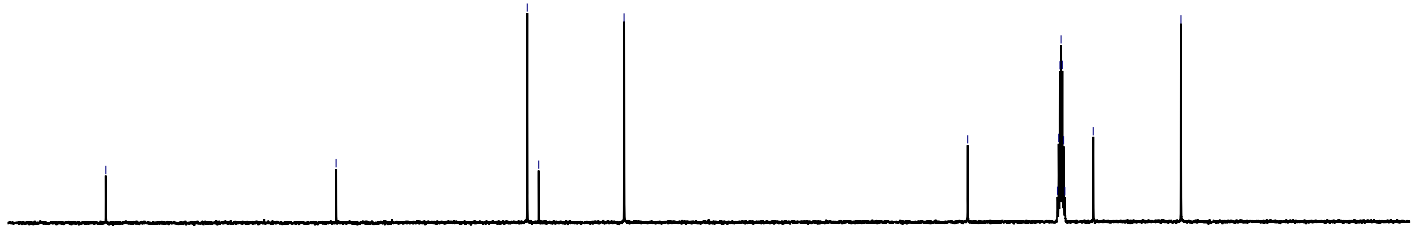

$\begin{array}{lllllllllllllllllllllll}210 & 200 & 190 & 180 & 170 & 160 & 150 & 140 & 130 & 120 & 110 & 100 & 90 & 80 & 70 & 60 & 50 & 40 & 30 & 20 & 10 & 0 & -10\end{array}$

Figure S24. ${ }^{13} \mathrm{C}$ NMR of 2-methyl-1-phenylbutan-1-one 


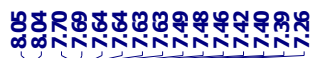
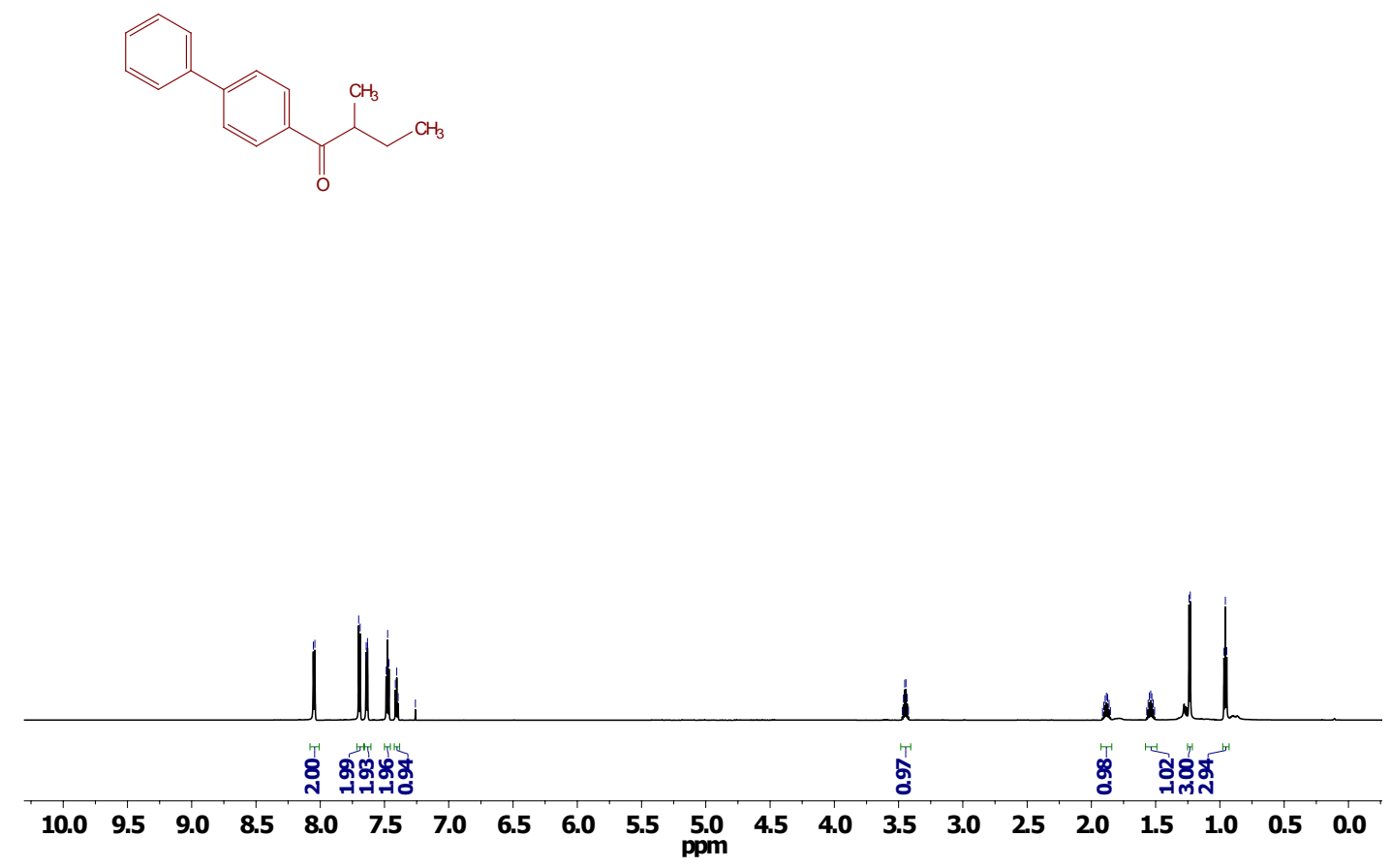

Figure S25. ${ }^{1} \mathrm{H}$ NMR of 1-([1,1'-biphenyl]-4-yl)-2-methylbutan-1-one
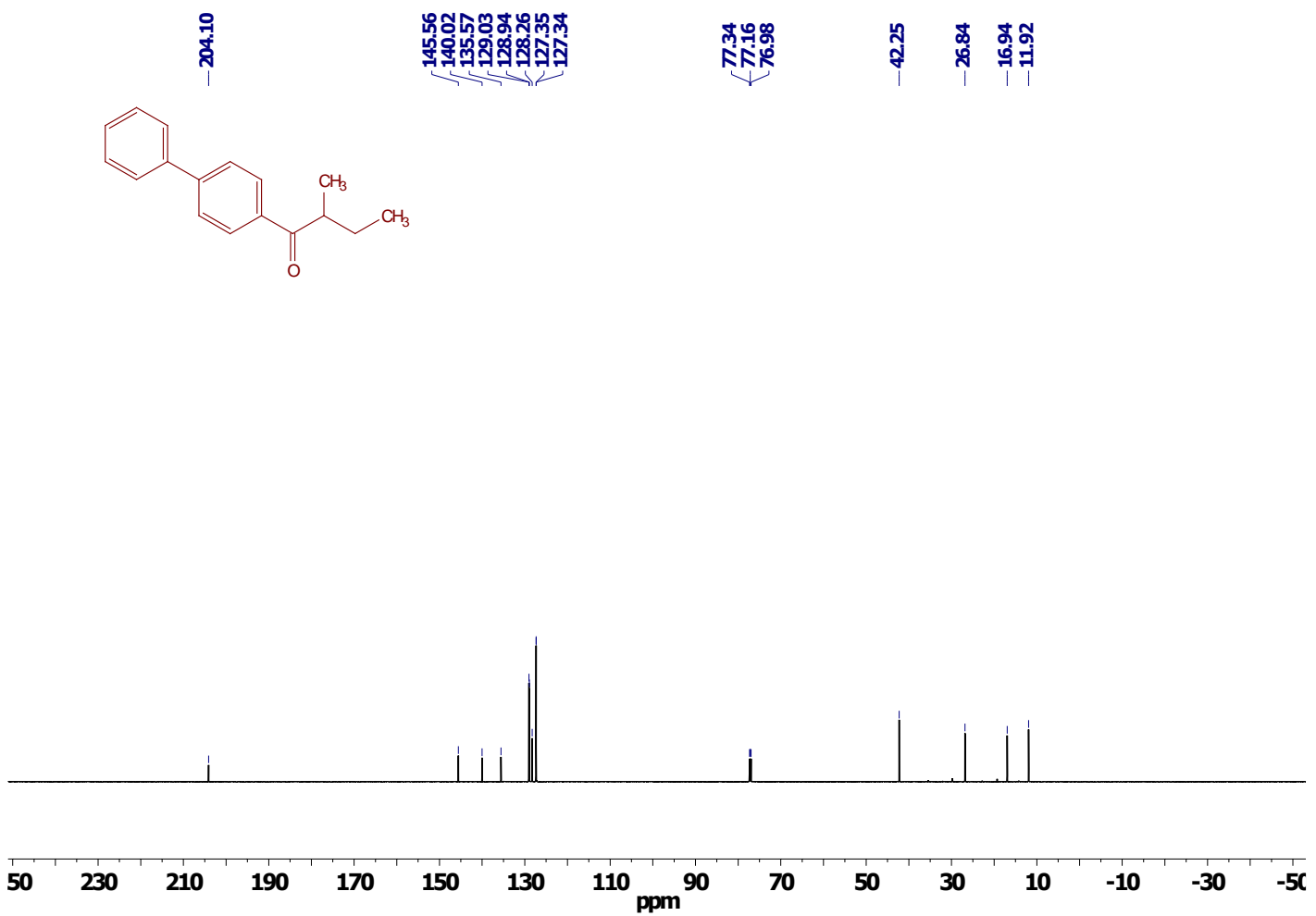

Figure S26. ${ }^{13} \mathrm{C}$ NMR of 1-([1,1'-biphenyl]-4-yl)-2-methylbutan-1-one 
<smiles>COc1cc2c(cc1OC)C(=O)C(O)C2</smiles>

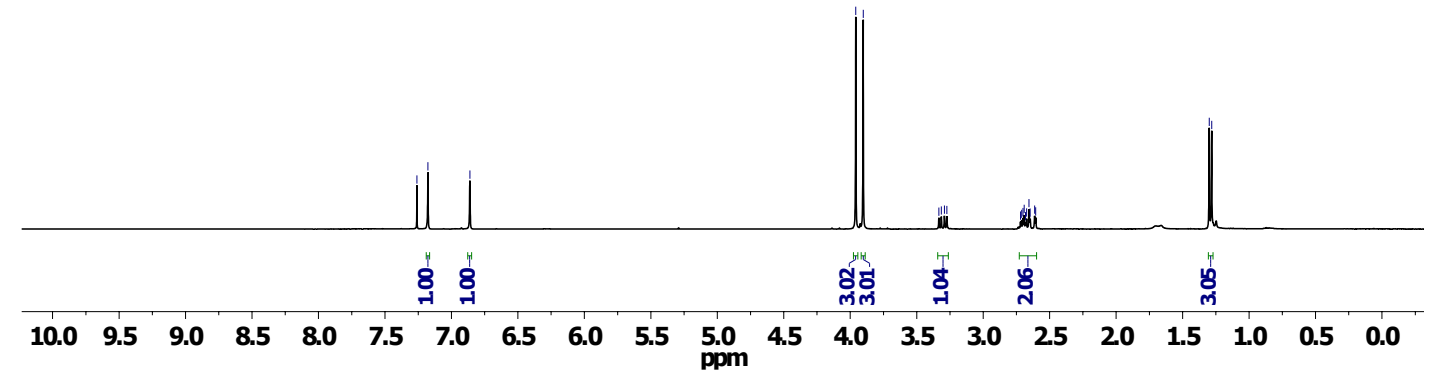

Figure S27. ${ }^{1} \mathrm{H}$ NMR of 5,6-dimethoxy-2-methyl-2,3-dihydro-1H-inden-1-one
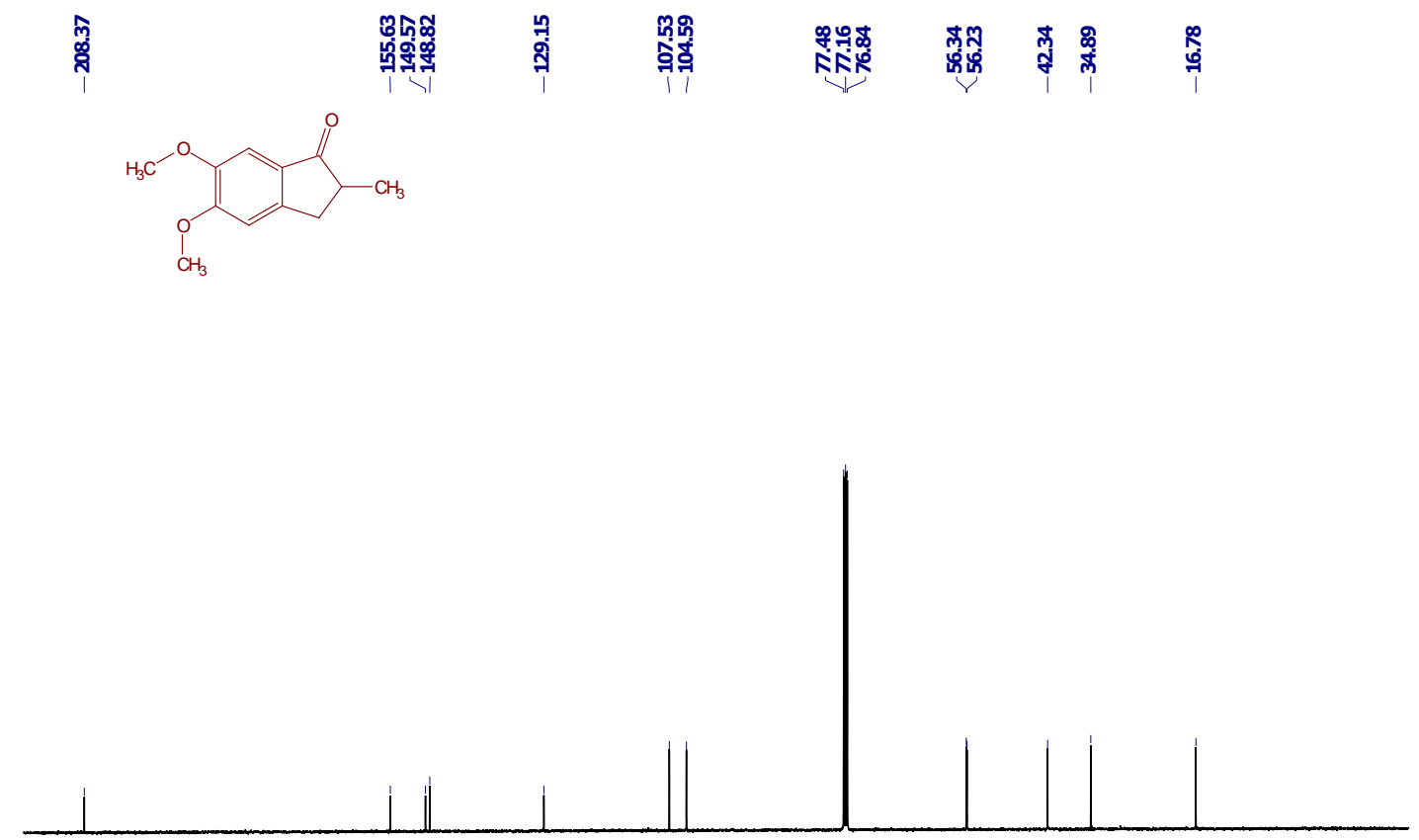

$\begin{array}{lllllllllllllllllllllll}210 & 200 & 190 & 180 & 170 & 160 & 150 & 140 & 130 & 120 & 110 & 100 & 90 & 80 & 70 & 60 & 50 & 40 & 30 & 20 & 10 & 0 & -10\end{array}$

Figure S28. ${ }^{13} \mathrm{C}$ NMR of 5,6-dimethoxy-2-methyl-2,3-dihydro-1H-inden-1-one 


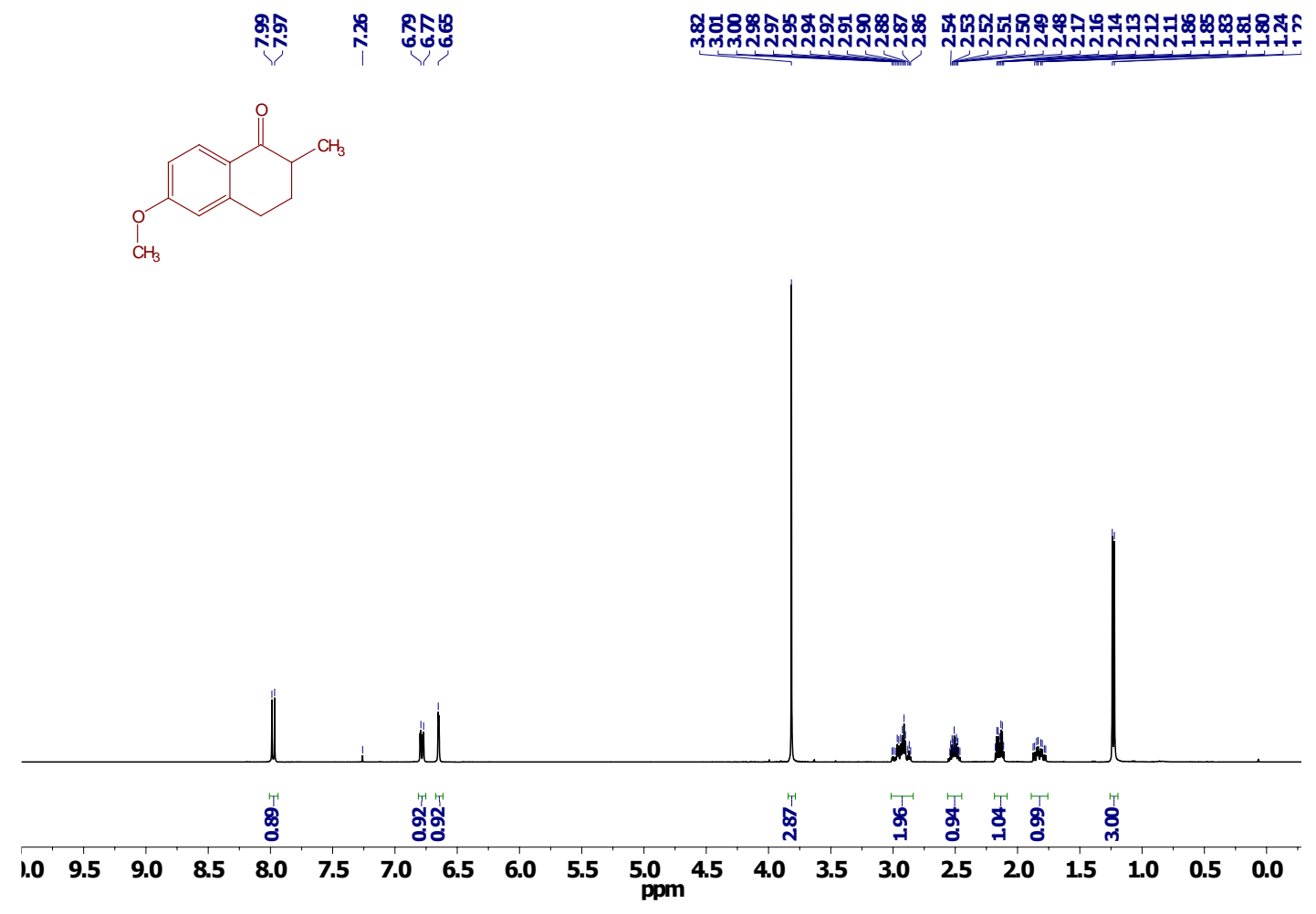

Figure S29. ${ }^{1} \mathrm{H}$ NMR of 6-methoxy-2-methyl-3,4-dihydronaphthalen-1(2H)-one

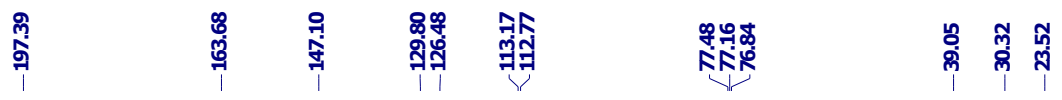<smiles>COc1ccc2c(c1)CCC(O)C2=O</smiles>

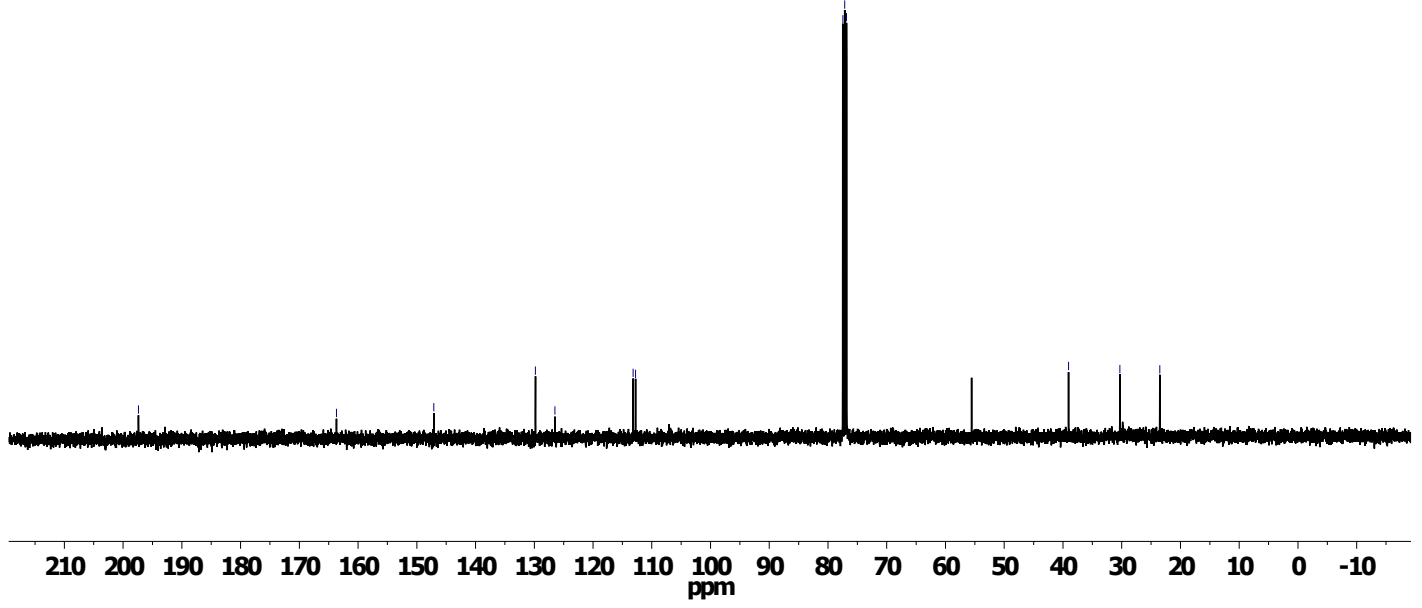

Figure S30. ${ }^{13} \mathrm{C}$ NMR of 6-methoxy-2-methyl-3,4-dihydronaphthalen-1(2H)-one 


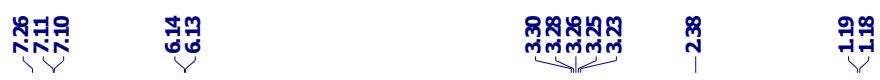<smiles>Cc1ccc(C(=O)C(C)C)o1</smiles>

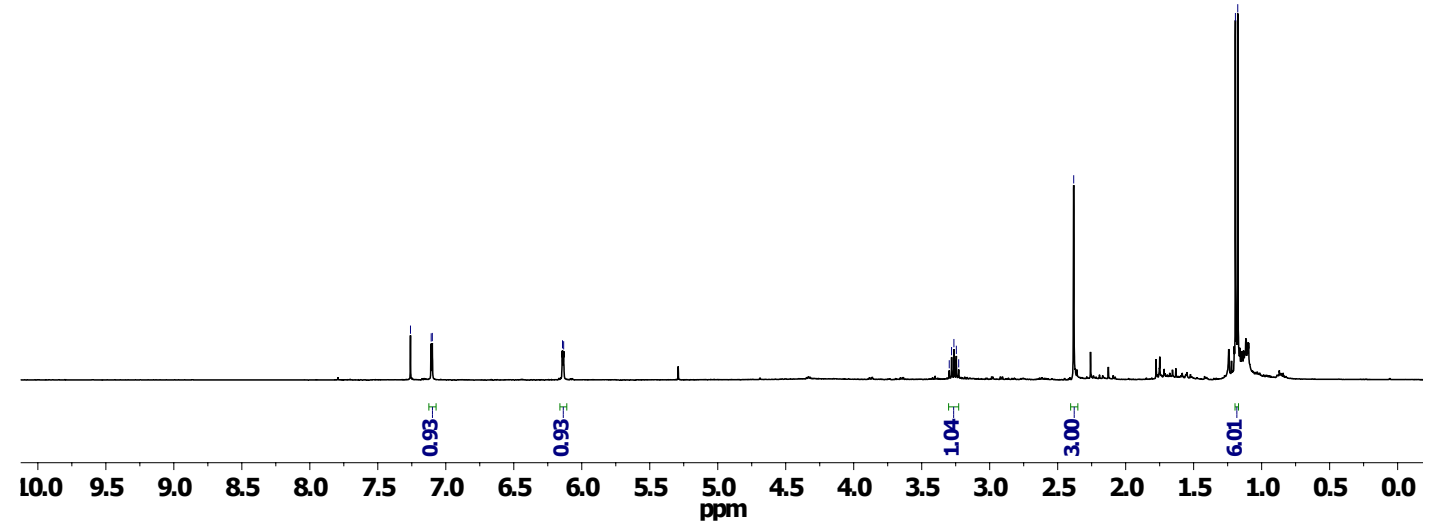

Figure S31. ${ }^{1} \mathrm{H}$ NMR of 2-methyl-1-(5-methylfuran-2-yl)propan-1-one

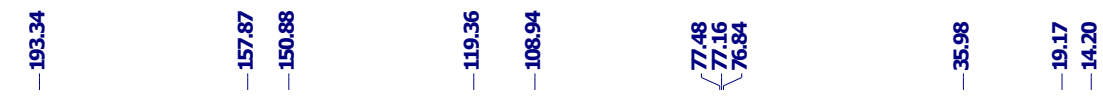<smiles>Cc1ccc(C(=O)C(C)C)o1</smiles>

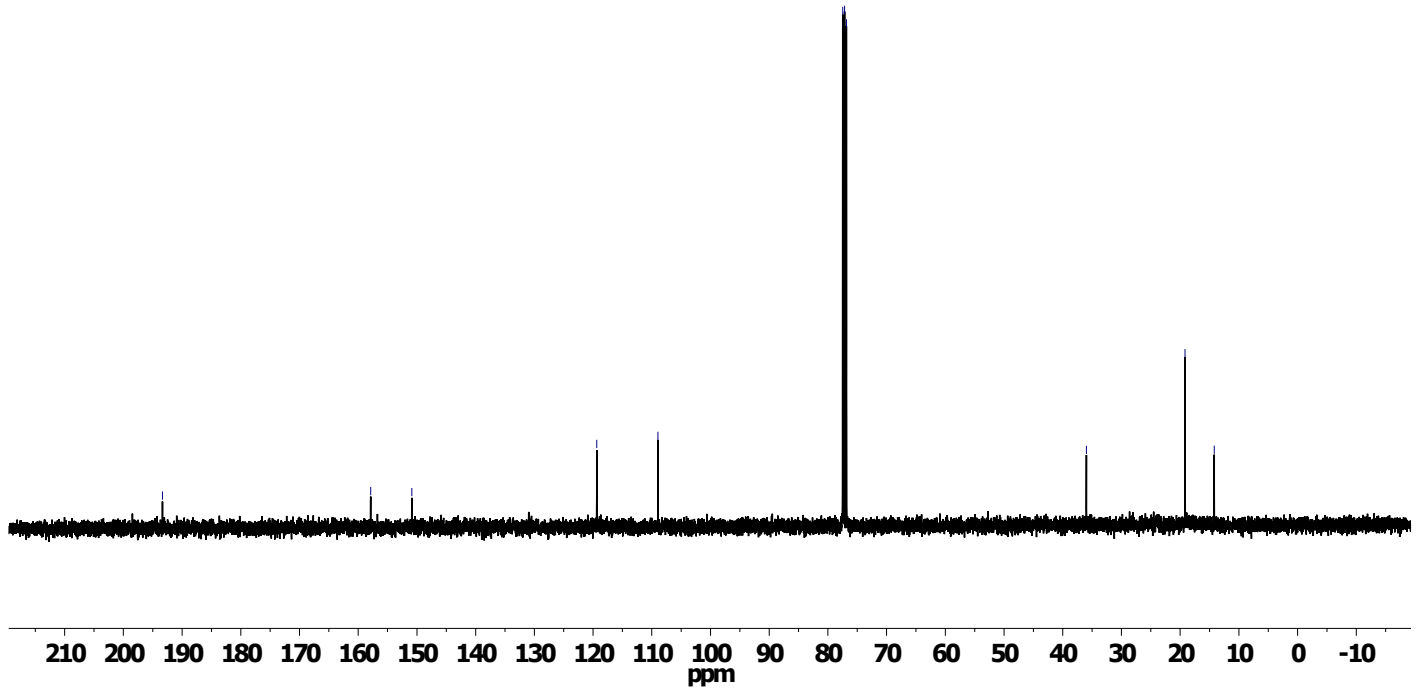

Figure S32. ${ }^{13} \mathrm{C}$ NMR of 2-methyl-1-(5-methylfuran-2-yl)propan-1-one 


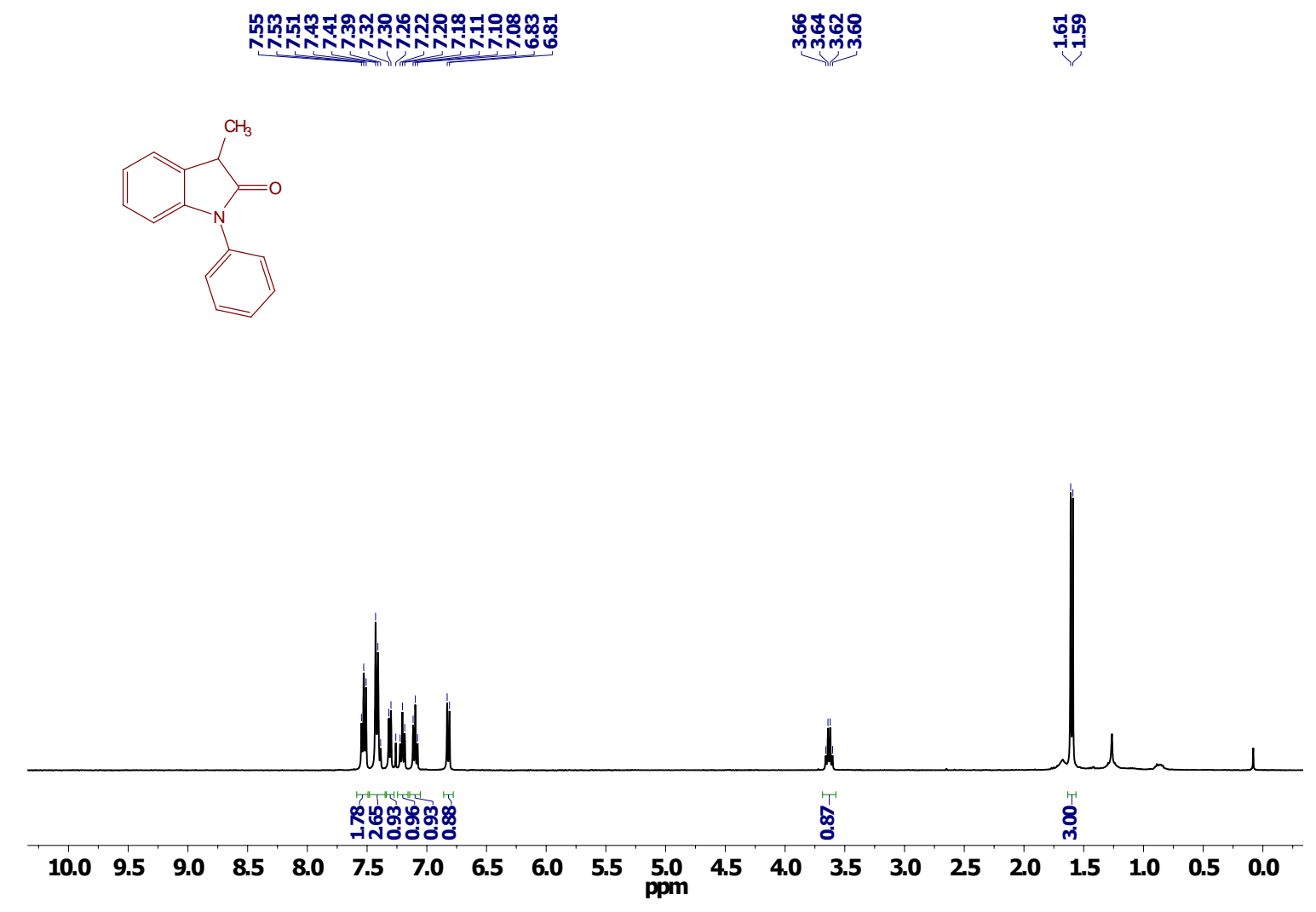

Figure S33. ${ }^{1} \mathrm{H}$ NMR of 3-methyl-1-phenylindolin-2-one

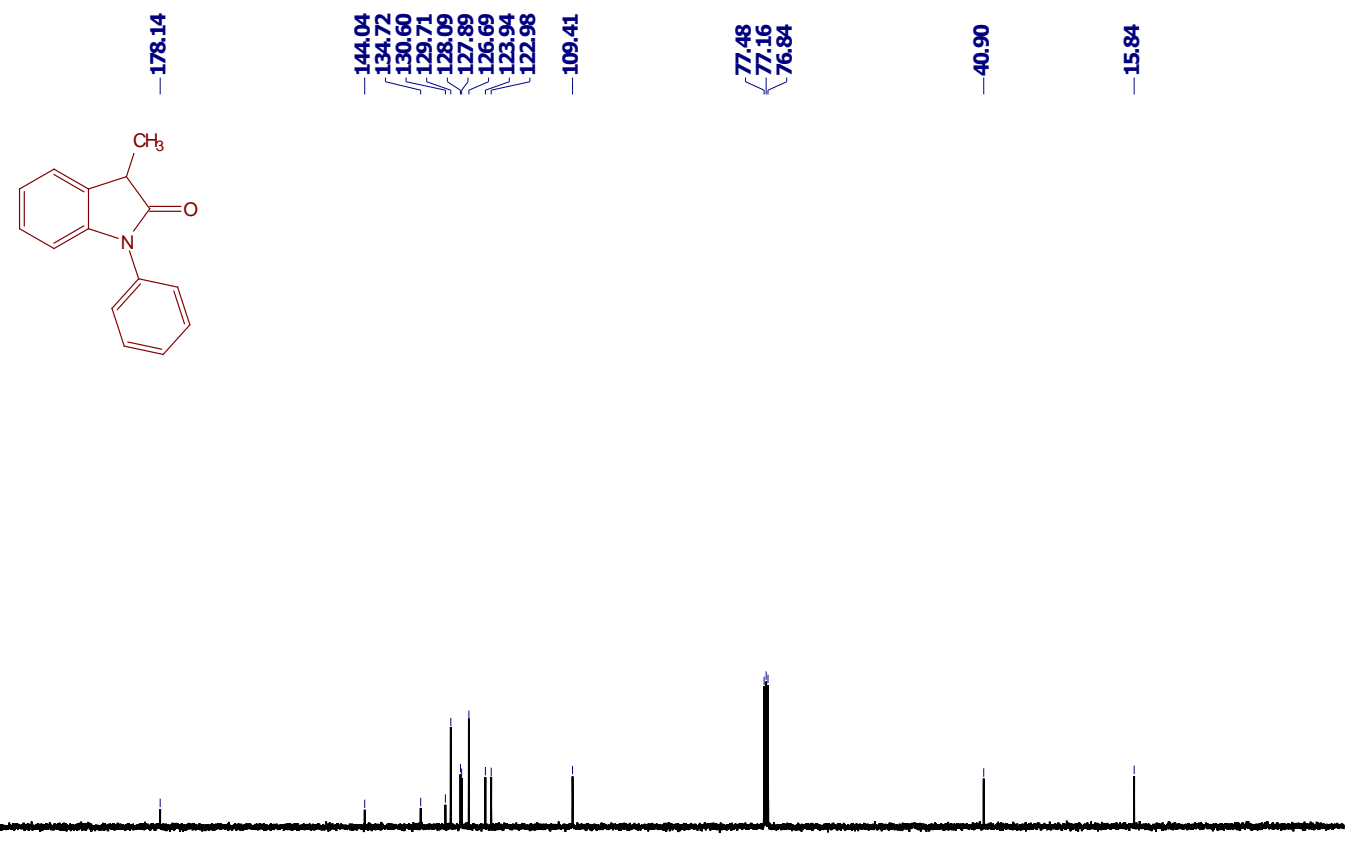

$\begin{array}{llllllllllllllllllllllll}210 & 200 & 190 & 180 & 170 & 160 & 150 & 140 & 130 & 120 & 110 & 100 & 90 & 80 & 70 & 60 & 50 & 40 & 30 & 20 & 10 & 0 & -10\end{array}$

Figure S34. ${ }^{13} \mathrm{C}$ NMR of 3-methyl-1-phenylindolin-2-one 

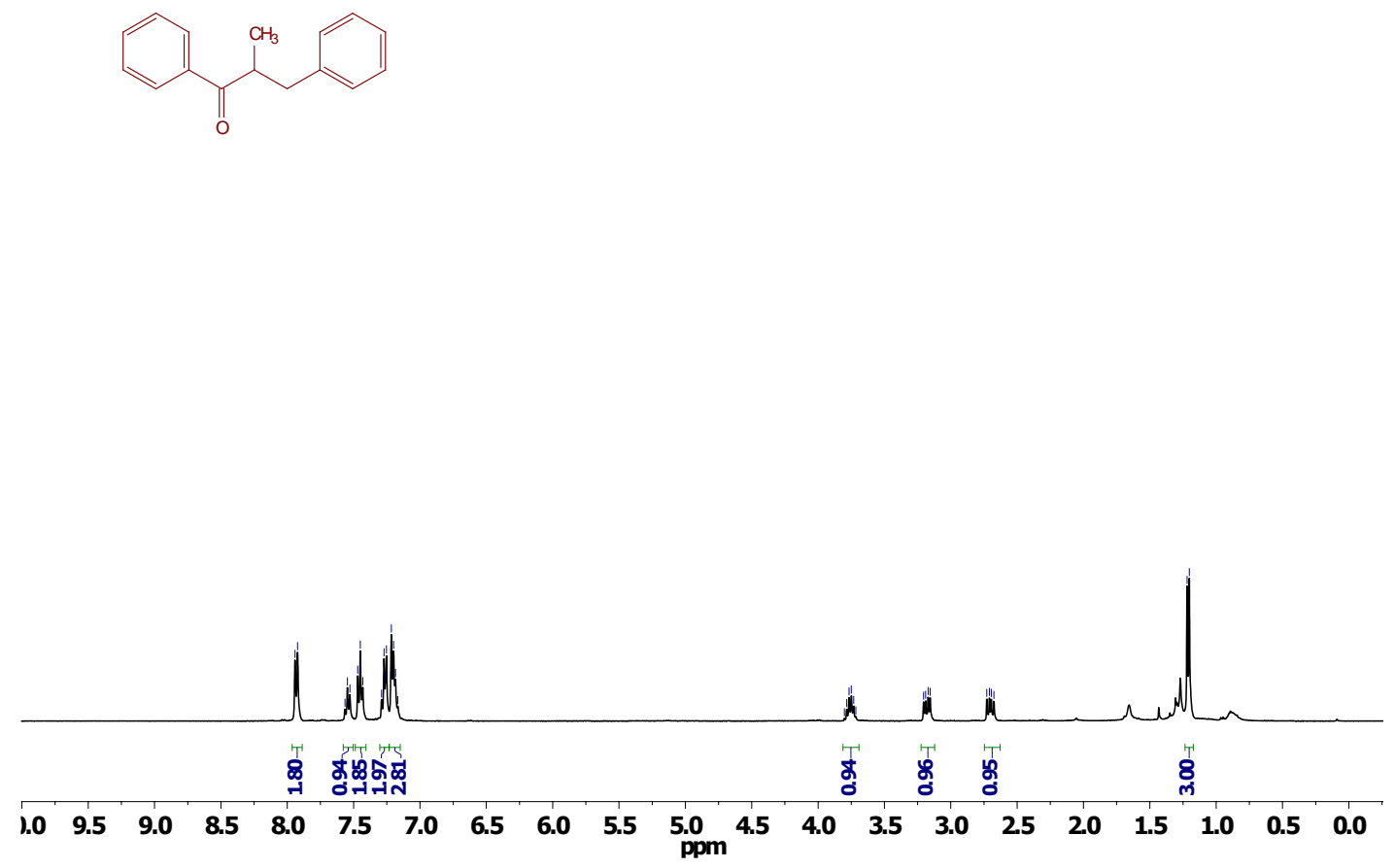

Figure S35. ${ }^{1} \mathrm{H}$ NMR of 2-methyl-1,3-diphenylpropan-1-one

ڤ్రై
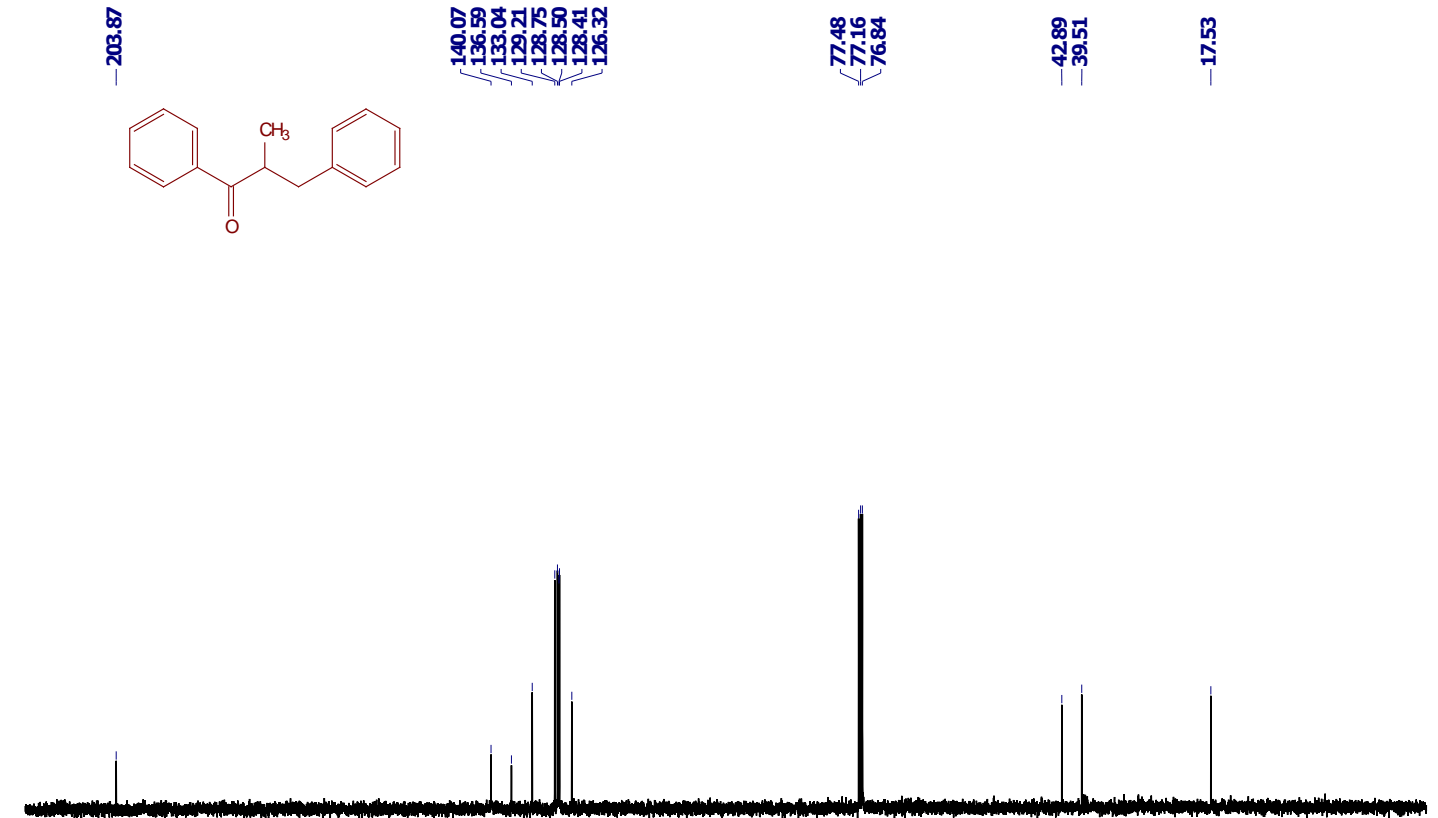

$\begin{array}{lllllllllllllllllllllll}210 & 200 & 190 & 180 & 170 & 160 & 150 & 140 & 130 & 120 & 110 & \begin{array}{c}100 \\ \mathrm{ppm}\end{array} & 90 & 80 & 70 & 60 & 50 & 40 & 30 & 20 & 10 & 0 & -10\end{array}$

Figure S36. ${ }^{13} \mathrm{C}$ NMR of 2-methyl-1,3-diphenylpropan-1-one 

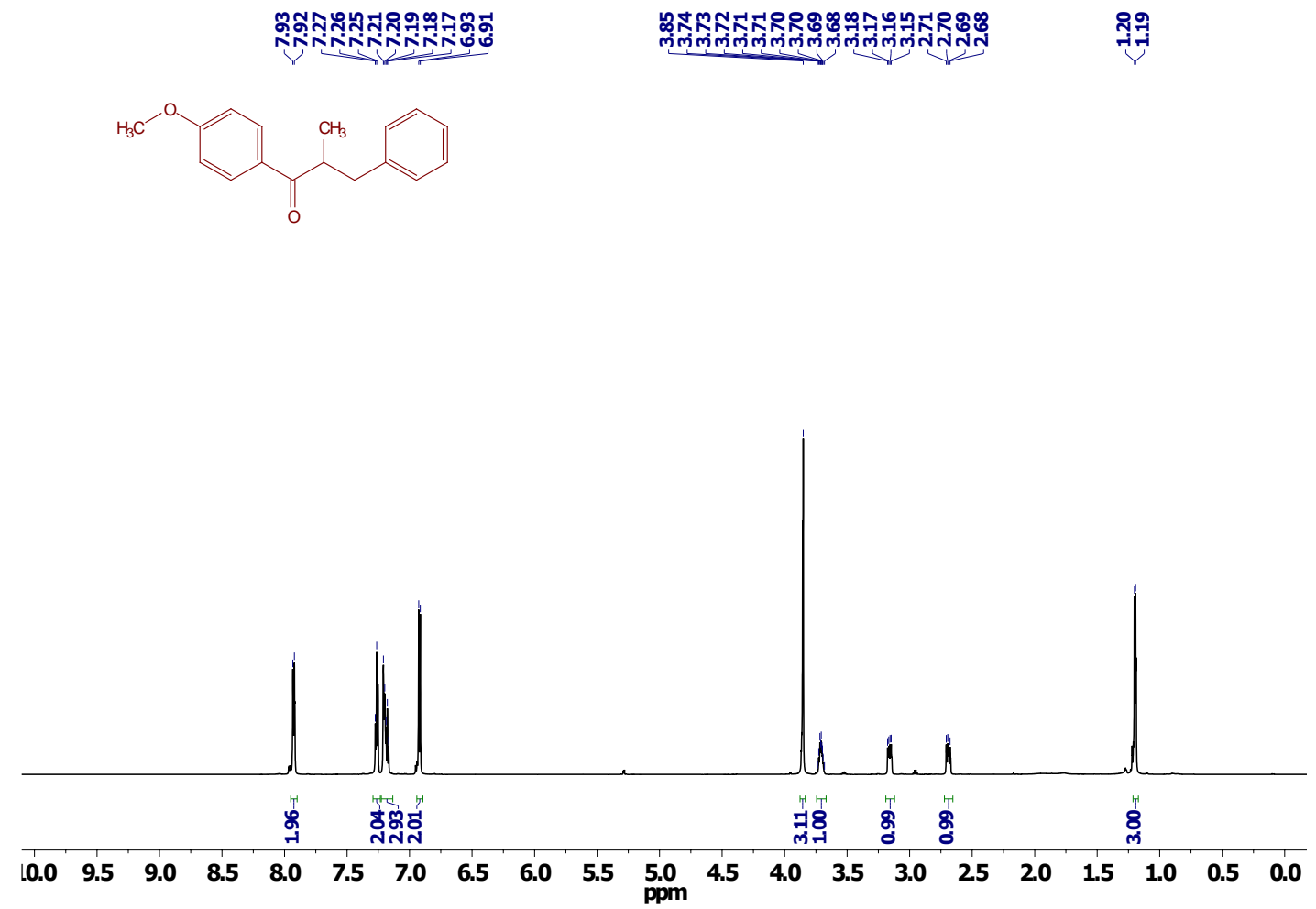

Figure S37. ${ }^{1} \mathrm{H}$ NMR of 1-(4-methoxyphenyl)-2-methyl-3-phenylpropan-1-one
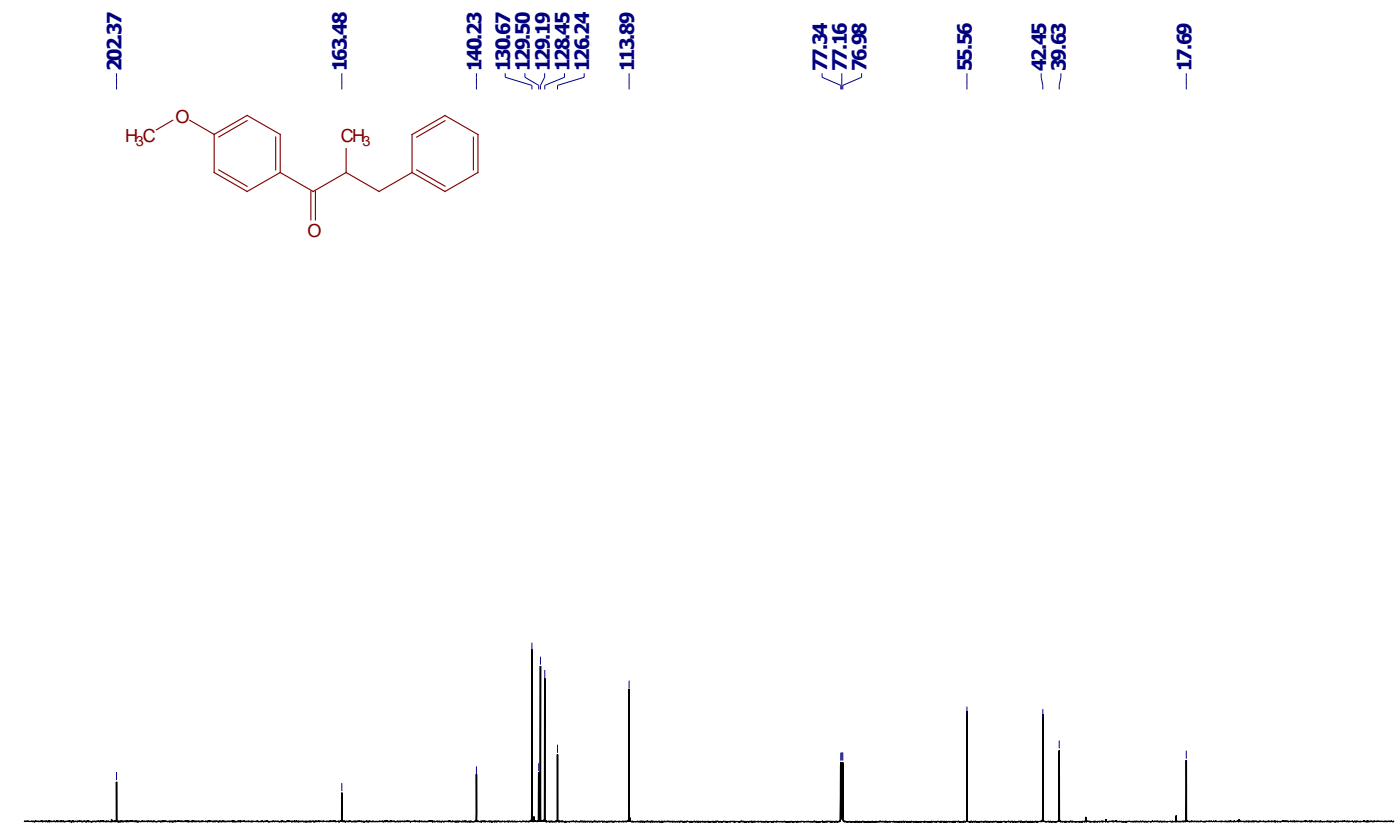

$\begin{array}{lllllllllllllllllllllll}210 & 200 & 190 & 180 & 170 & 160 & 150 & 140 & 130 & 120 & 110 & \begin{array}{c}100 \\ \mathrm{ppm}\end{array} & 90 & 80 & 70 & 60 & 50 & 40 & 30 & 20 & 10 & 0 & -10\end{array}$

Figure S38. ${ }^{13} \mathrm{C}$ NMR of 1-(4-methoxyphenyl)-2-methyl-3-phenylpropan-1-one 

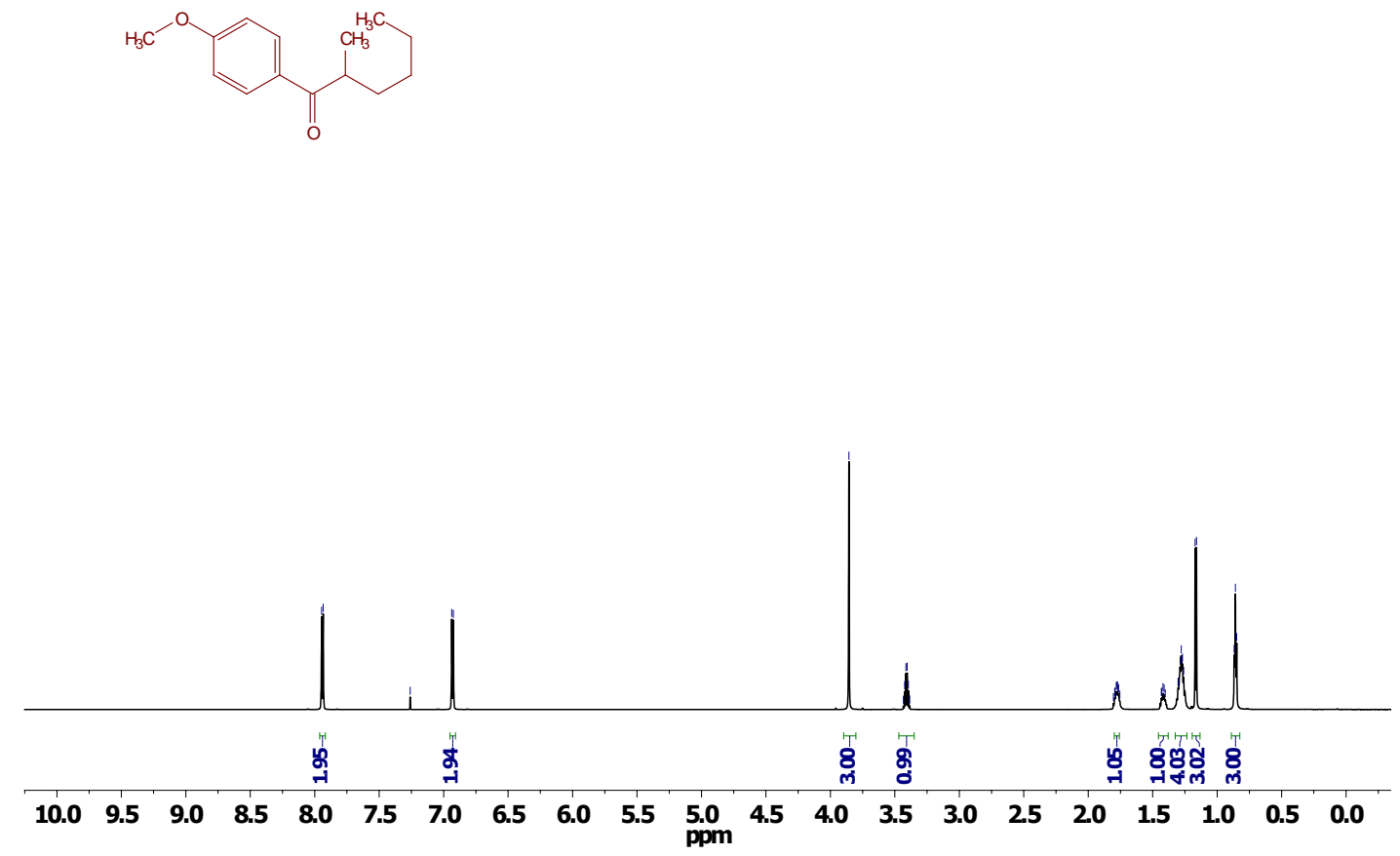

Figure S39. ${ }^{1} \mathrm{H}$ NMR of 1-(4-methoxyphenyl)-2-methylhexan-1-one
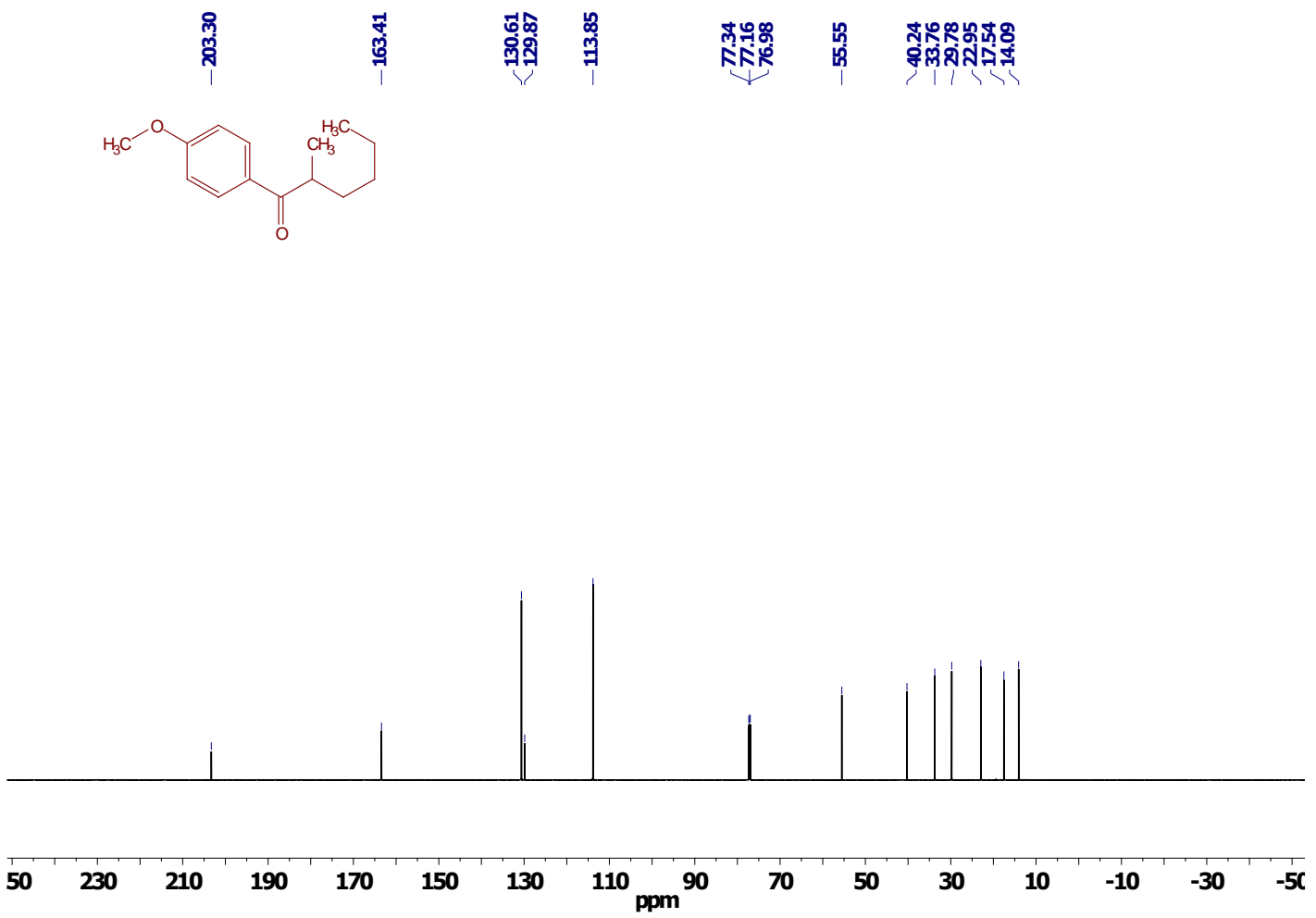

Figure S40. ${ }^{13} \mathrm{C}$ NMR of 1-(4-methoxyphenyl)-2-methylhexan-1-one 


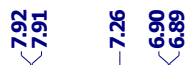
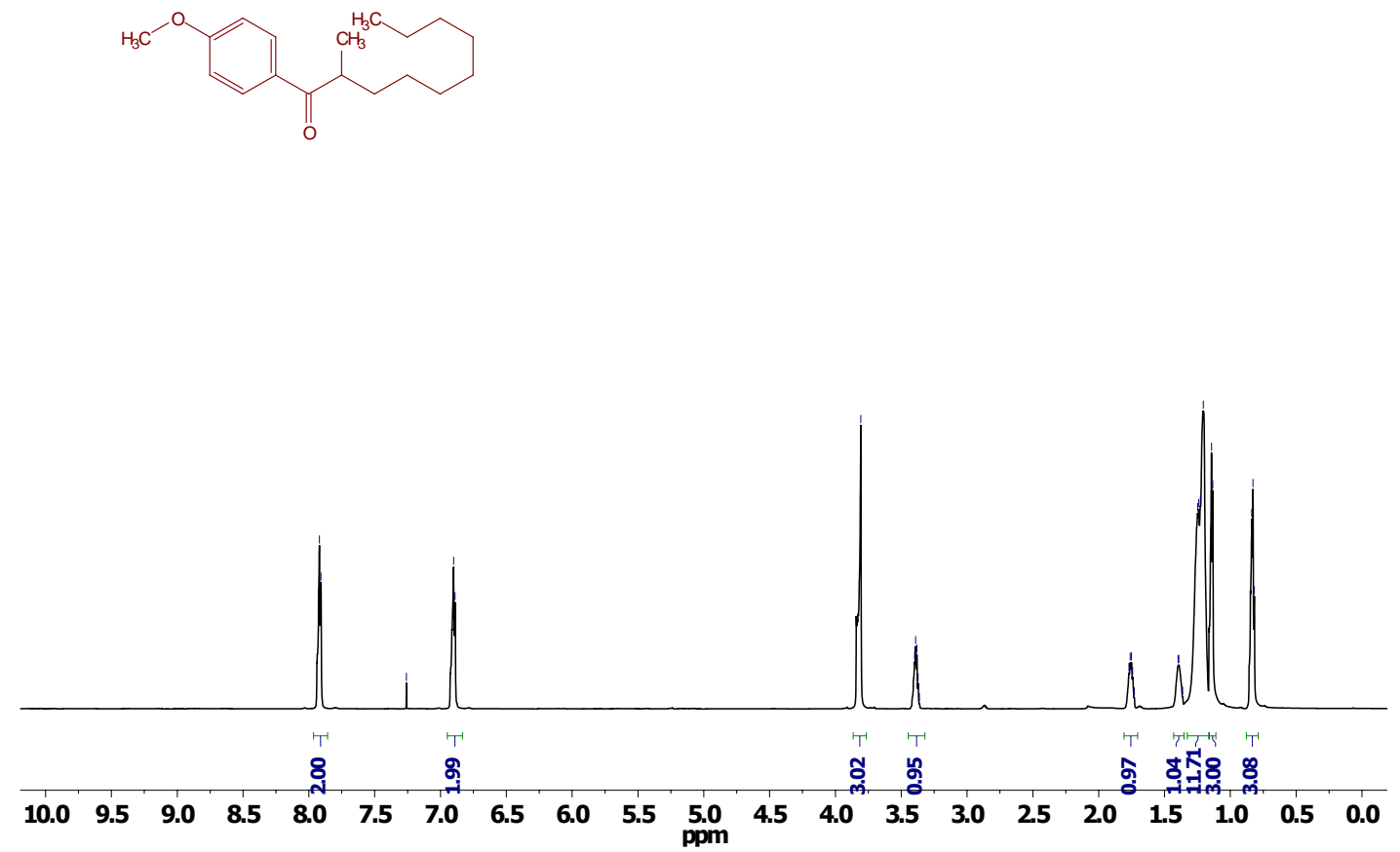

Figure S41. ${ }^{1} \mathrm{H}$ NMR of 1-(4-methoxyphenyl)-2-methyldecan-1-one
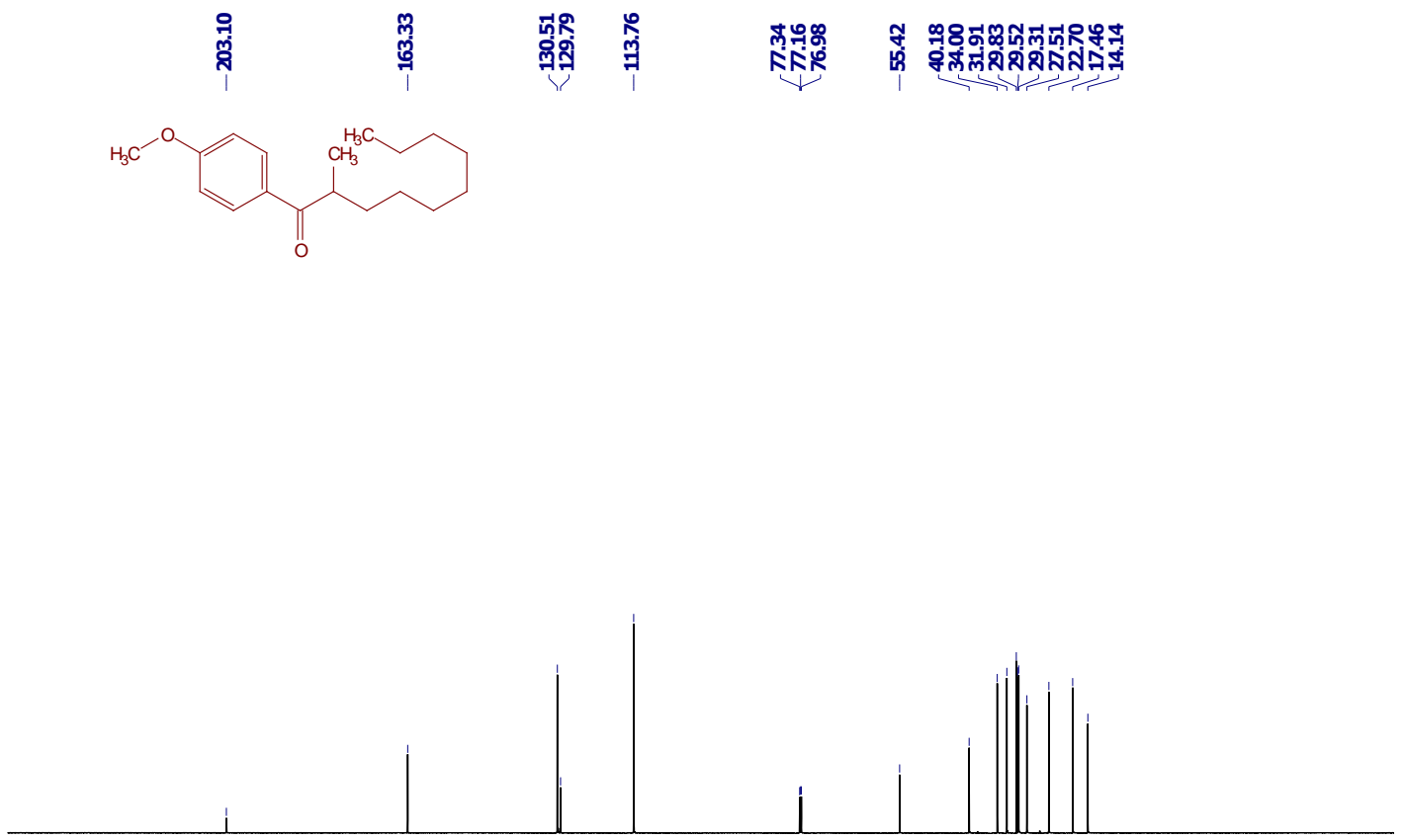

$\begin{array}{llllllllllllllllllllllllll}50 & 230 & 210 & 190 & 170 & 150 & 130 & 110 & 90 & 70 & 50 & 30 & 10 & -10 & -30 & -5 C\end{array}$

Figure S42. ${ }^{13} \mathrm{C}$ NMR of 1-(4-methoxyphenyl)-2-methyldecan-1-one 


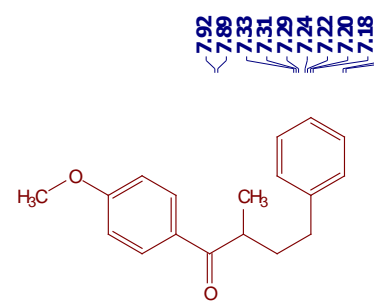

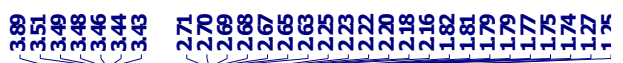

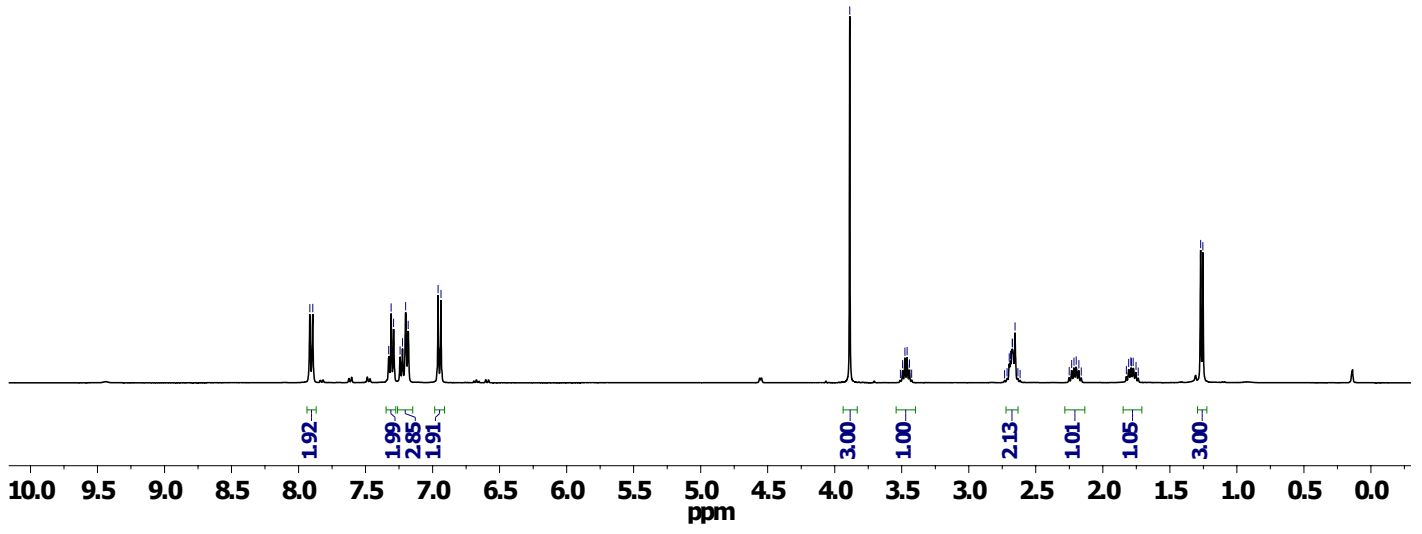

Figure S43. ${ }^{1} \mathrm{H}$ NMR of 1-(4-methoxyphenyl)-2-methyl-4-phenylbutan-1-one
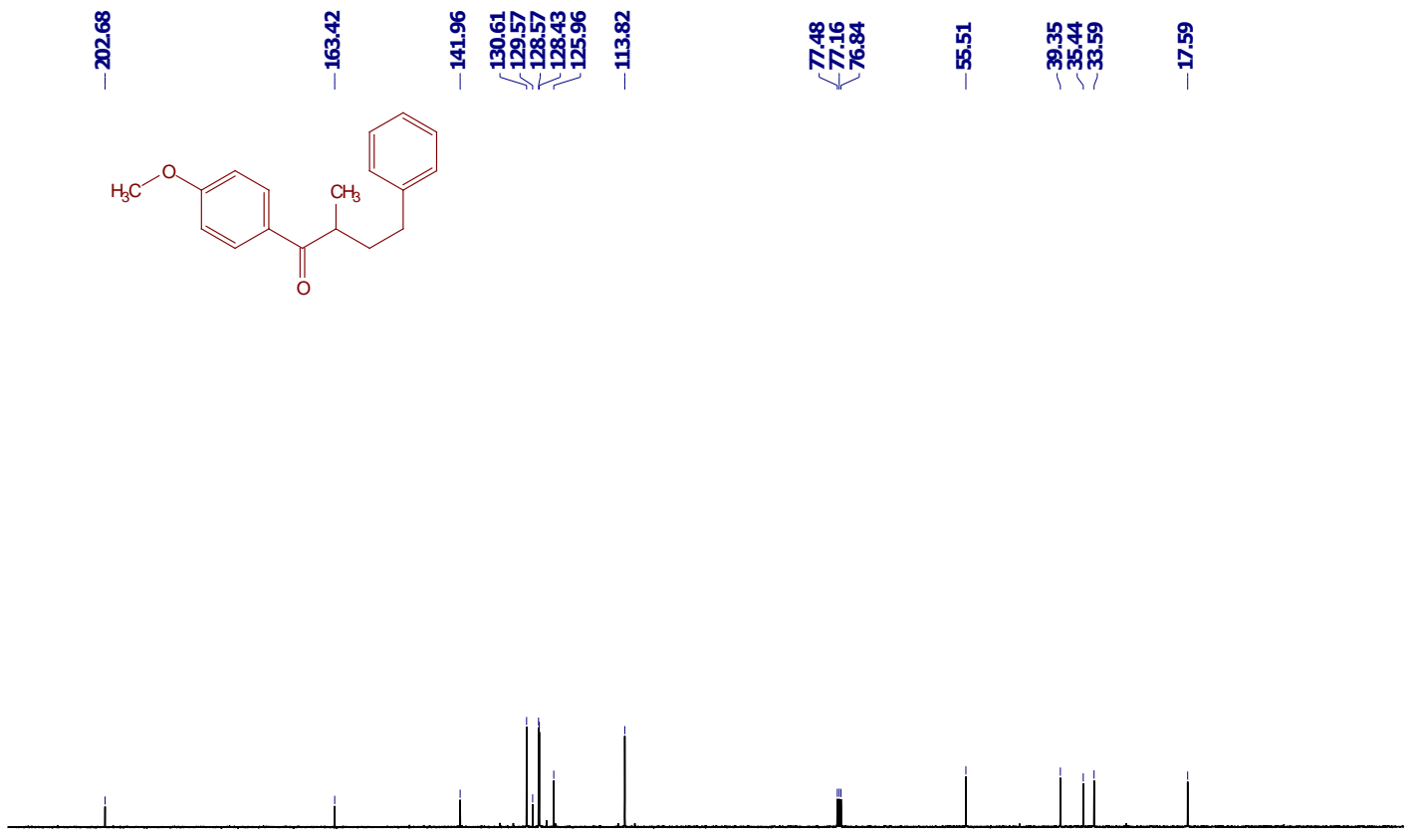

$\begin{array}{lllllllllllllllllllllllllll}210 & 200 & 190 & 180 & 170 & 160 & 150 & 140 & 130 & 120 & 110 & \mathbf{1 0 0} & 90 & 80 & 70 & 60 & 50 & 40 & 30 & 20 & 10 & 0 & -10\end{array}$

Figure S44. ${ }^{13} \mathrm{C}$ NMR of 1-(4-methoxyphenyl)-2-methyl-4-phenylbutan-1-one 


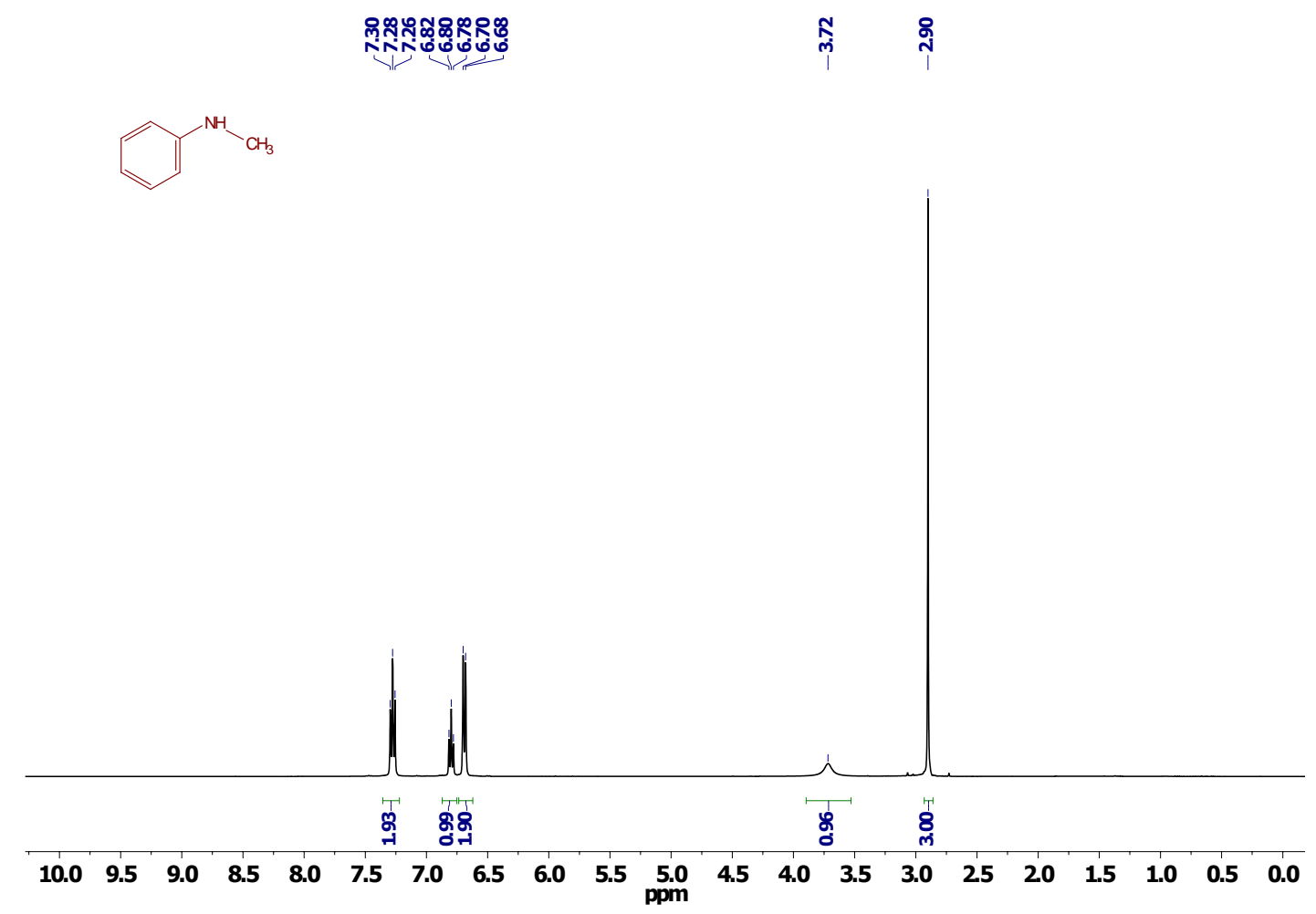

Figure S45. ${ }^{1} \mathrm{H}$ NMR of N-methylaniline
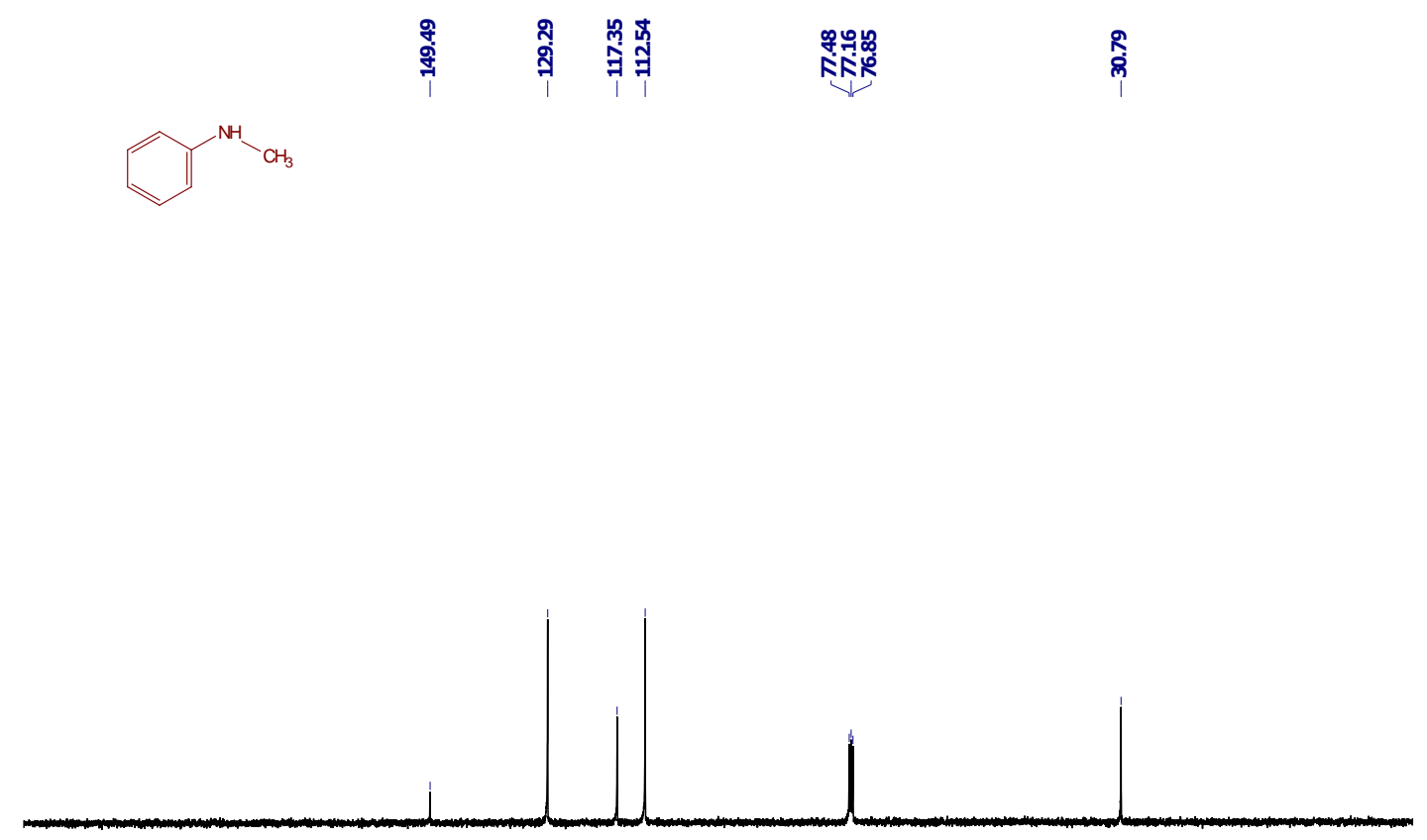

$\begin{array}{lllllllllllllllllllllll}210 & 200 & 190 & 180 & 170 & 160 & 150 & 140 & 130 & 120 & 110 & 100 & 90 & 80 & 70 & 60 & 50 & 40 & 30 & 20 & 10 & 0 & -10\end{array}$

Figure S46. ${ }^{13} \mathrm{C}$ NMR of $\mathrm{N}$-methylaniline 

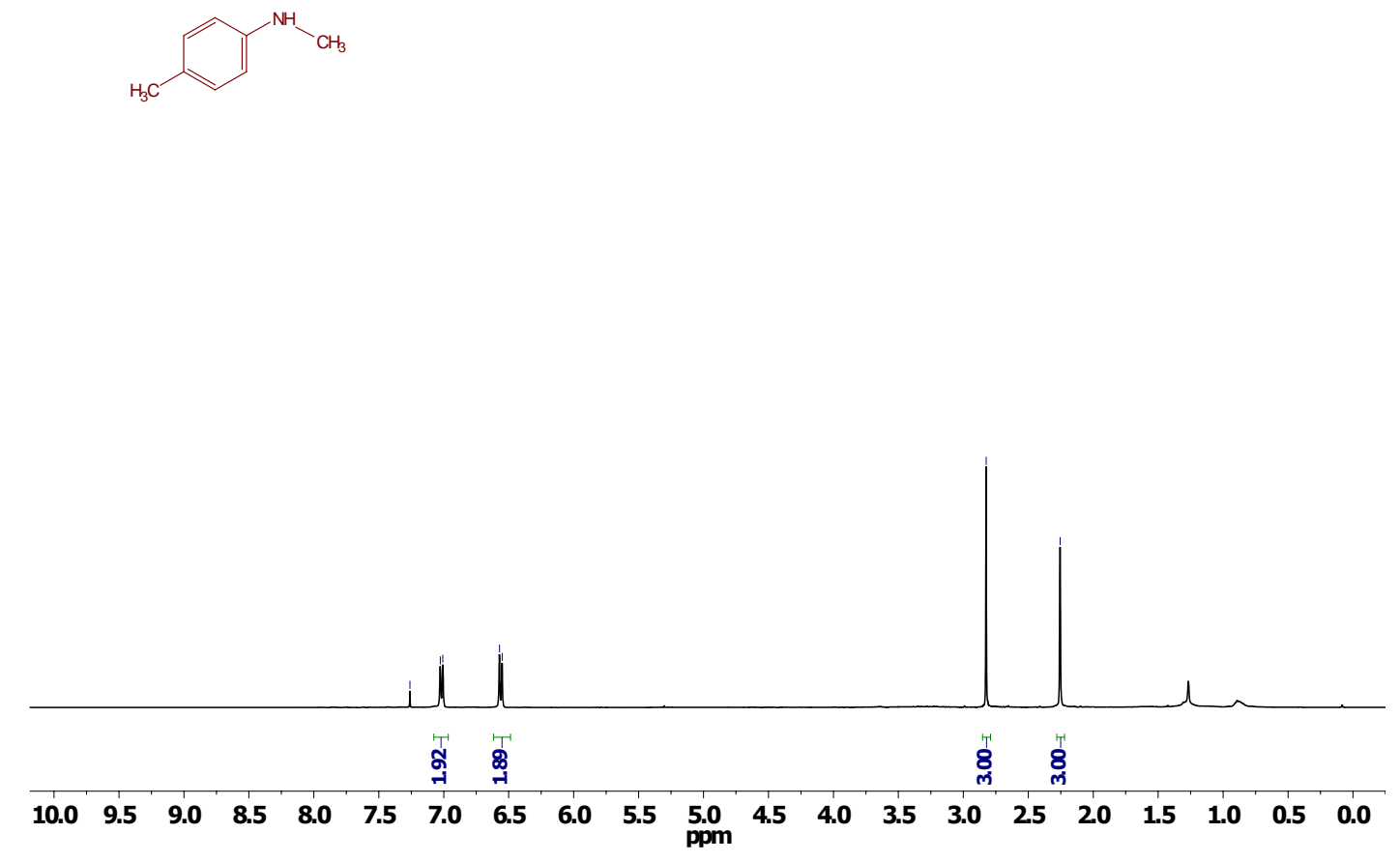

Figure S47. ${ }^{1} \mathrm{H}$ NMR of N,4-dimethylaniline

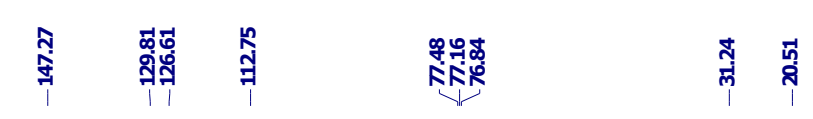<smiles>Cc1ccc(NO)cc1</smiles>

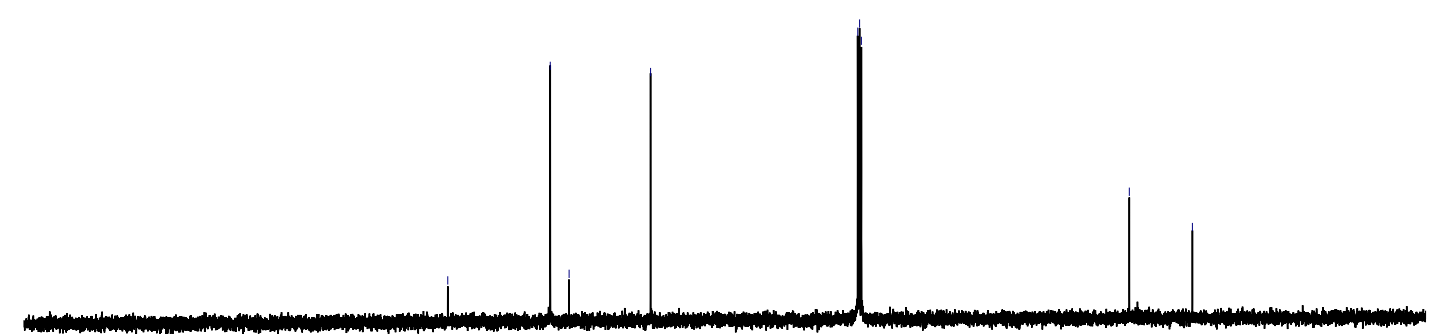

$\begin{array}{lllllllllllllllllllllllll}210 & 200 & 190 & 180 & 170 & 160 & 150 & 140 & 130 & 120 & 110 & \mathbf{1 0 0} & 90 & 80 & 70 & 60 & 50 & 40 & 30 & 20 & 10 & 0 & -10\end{array}$

Figure S48. ${ }^{13} \mathrm{C}$ NMR of N,4-dimethylaniline 
$\mathrm{CO}_{\mathrm{CH}_{3}}^{\mathrm{NH}}$

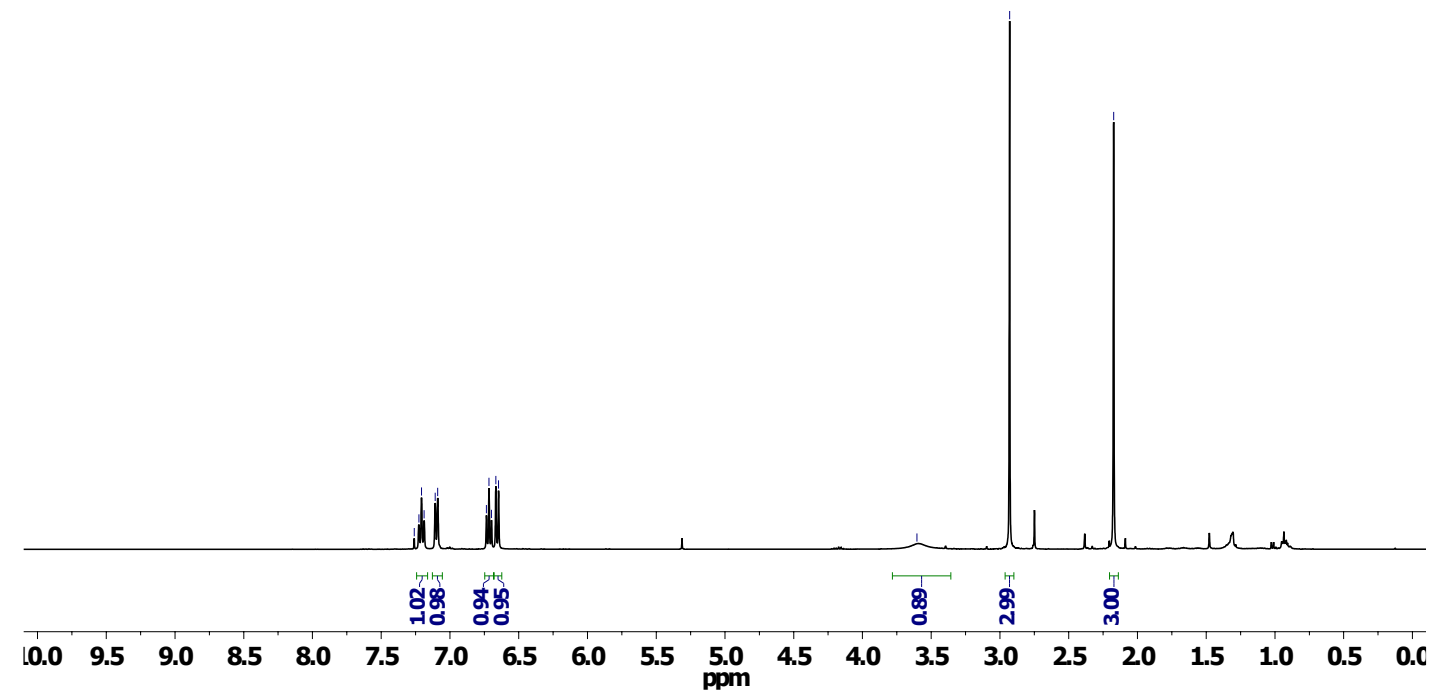

Figure S49. ${ }^{1} \mathrm{H}$ NMR of N,2-dimethylaniline

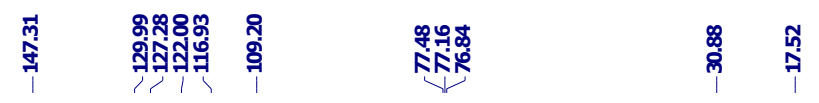<smiles>NOc1ccccc1O</smiles>

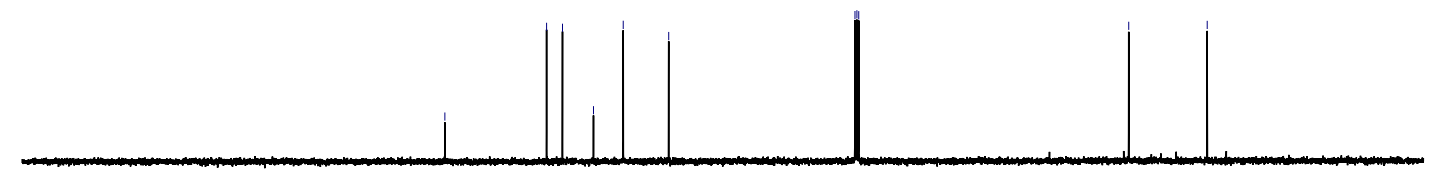

$\begin{array}{lllllllllllllllllllllll}210 & 200 & 190 & 180 & 170 & 160 & 150 & 140 & 130 & 120 & 110 & \begin{array}{c}100 \\ \mathrm{ppm}\end{array} & 90 & 80 & 70 & 60 & 50 & 40 & 30 & 20 & 10 & 0 & -10\end{array}$

Figure S50. ${ }^{13} \mathrm{C}$ NMR of N,2-dimethylaniline 

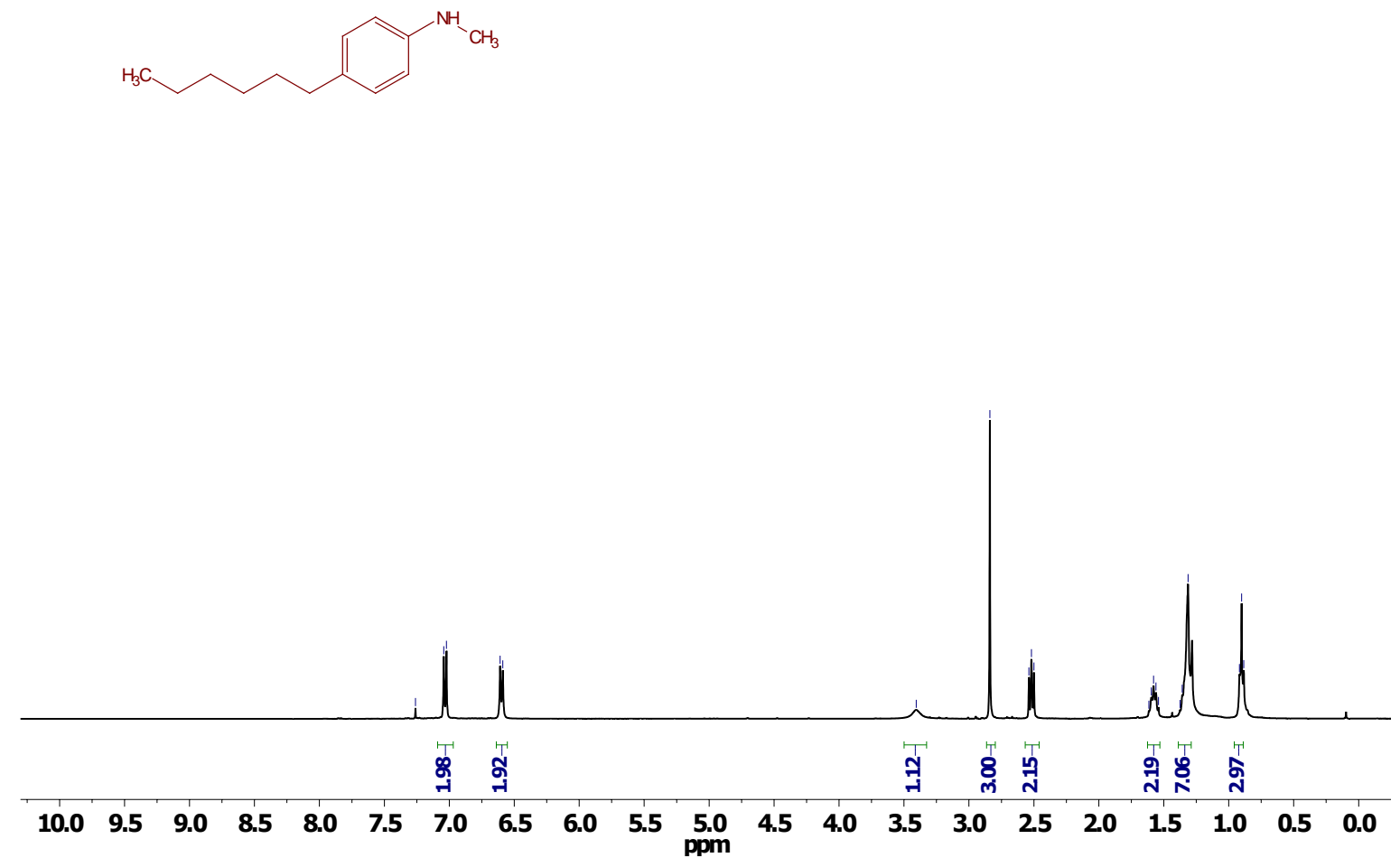

Figure S51. ${ }^{1} \mathrm{H}$ NMR of 4-hexyl-N-methylaniline

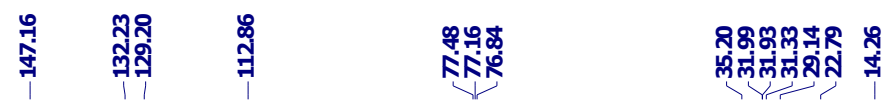<smiles>NNc1ccc(CCCCCO)cc1</smiles>

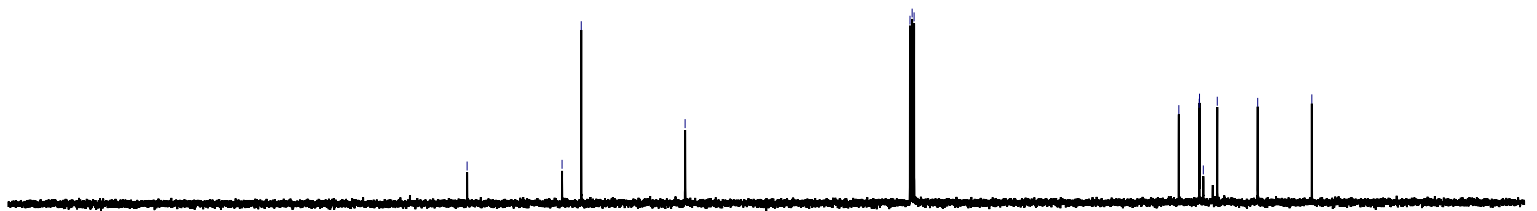

$\begin{array}{llllllllllllllllllllllll}210 & 200 & 190 & 180 & 170 & 160 & 150 & 140 & 130 & 120 & 110 & 100 & 90 & 80 & 70 & 60 & 50 & 40 & 30 & 20 & 10 & 0 & -10\end{array}$

Figure S52. ${ }^{13} \mathrm{C}$ NMR of 4-hexyl-N-methylaniline 


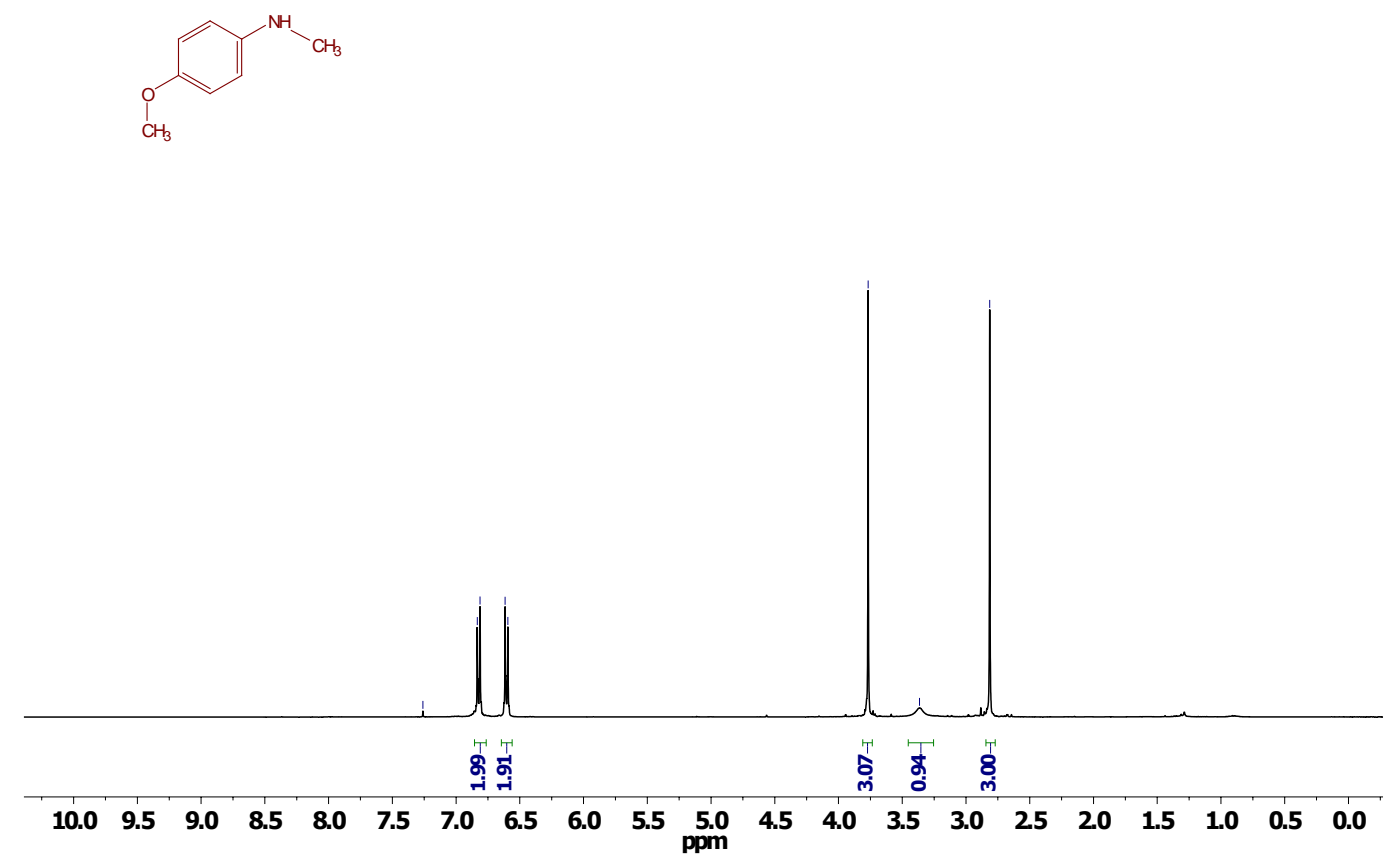

Figure S53. ${ }^{1} \mathrm{H}$ NMR of 4-methoxy-N-methylaniline

\begin{tabular}{|c|c|c|c|}
\hline 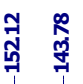 & $\begin{array}{l}\mathscr{R} R \\
\text { J̛̣ }\end{array}$ & 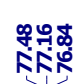 & 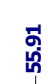 \\
\hline
\end{tabular}

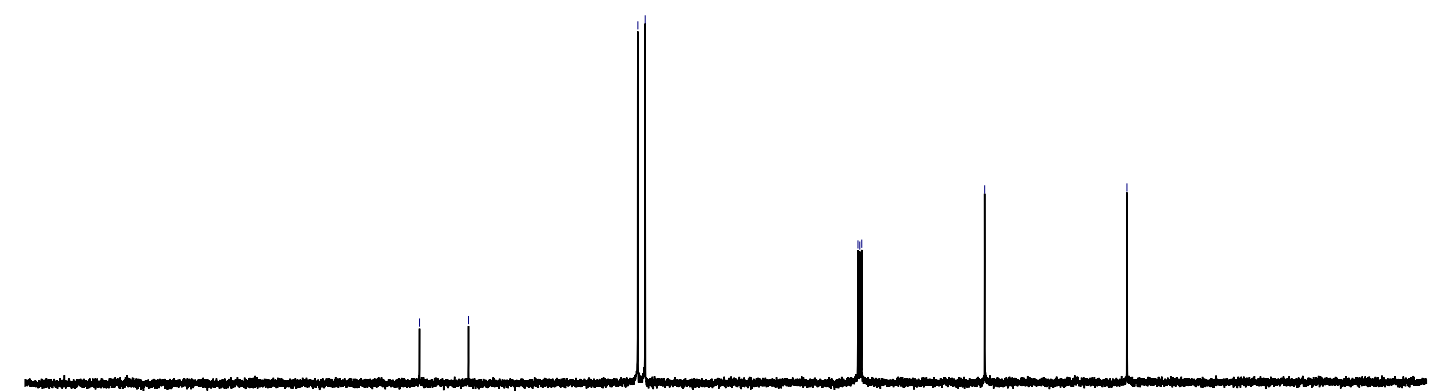

$\begin{array}{lllllllllllllllllllllllll}210 & 200 & 190 & 180 & 170 & 160 & 150 & 140 & 130 & 120 & 110 & \begin{array}{c}100 \\ \mathrm{ppm}\end{array} & 90 & 80 & 70 & 60 & 50 & 40 & 30 & 20 & 10 & 0 & -10\end{array}$

Figure S54. ${ }^{13} \mathrm{C}$ NMR of 4-methoxy-N-methylaniline 


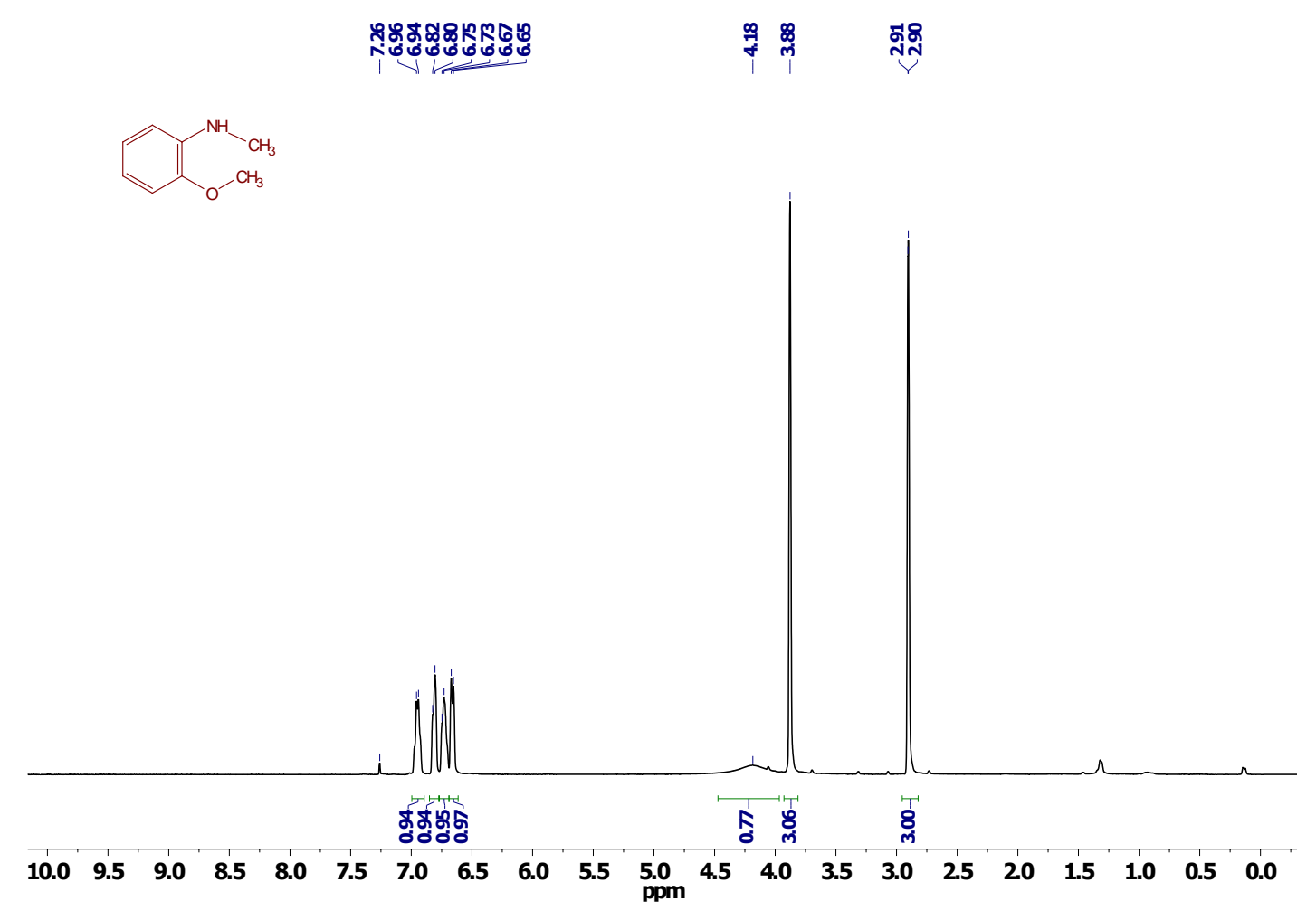

Figure S55. ${ }^{1} \mathrm{H}$ NMR of 2-methoxy-N-methylaniline

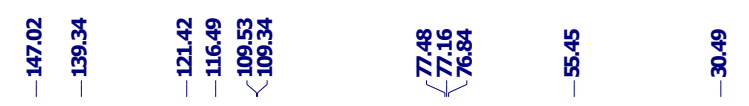

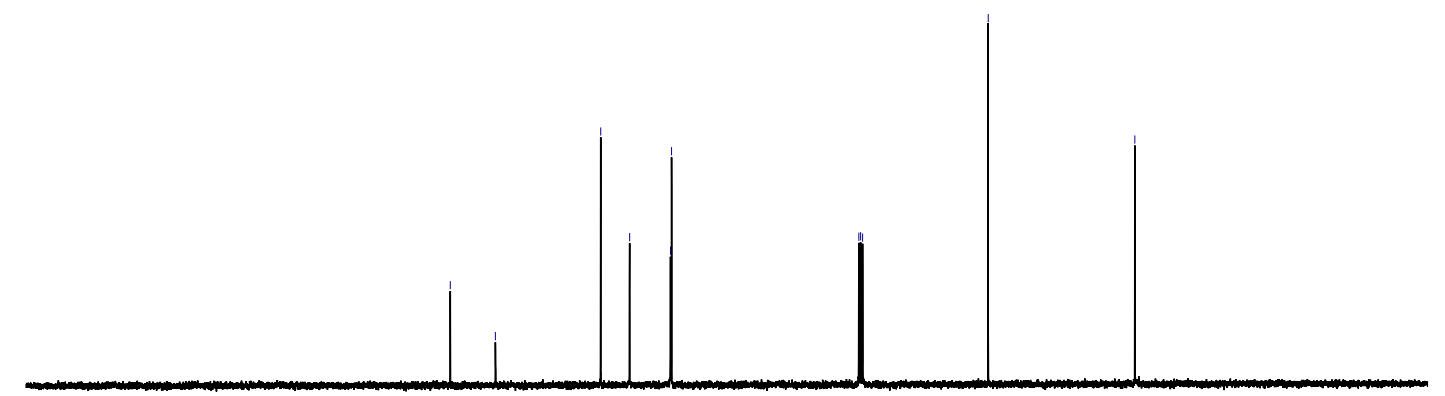

$\begin{array}{lllllllllllllllllllllllll}210 & 200 & 190 & 180 & 170 & 160 & 150 & 140 & 130 & 120 & 110 & \begin{array}{c}100 \\ \mathrm{ppm}\end{array} & 90 & 80 & 70 & 60 & 50 & 40 & 30 & 20 & 10 & 0 & -10\end{array}$

Figure S56. ${ }^{13} \mathrm{C}$ NMR of 2-methoxy-N-methylaniline 


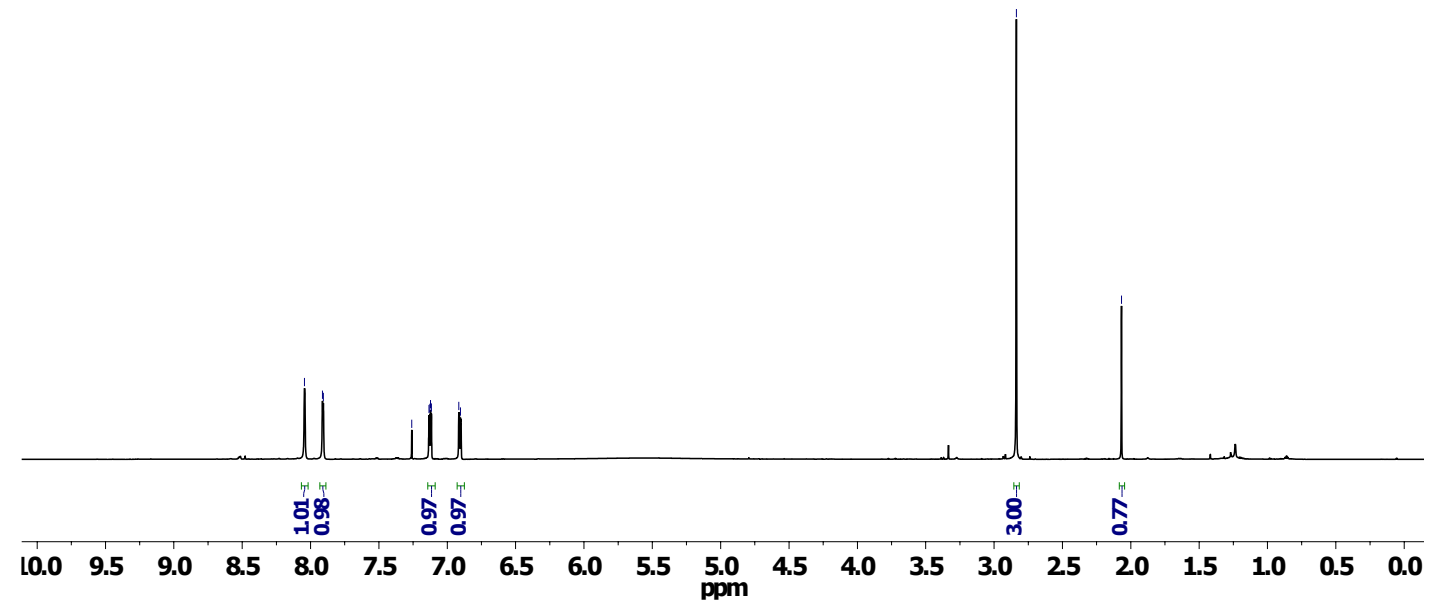

Figure S57. ${ }^{1} \mathrm{H}$ NMR of N-methylpyridin-3-amine

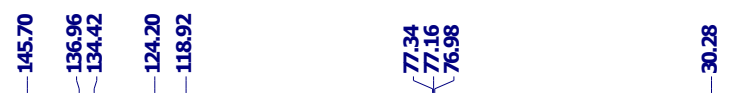

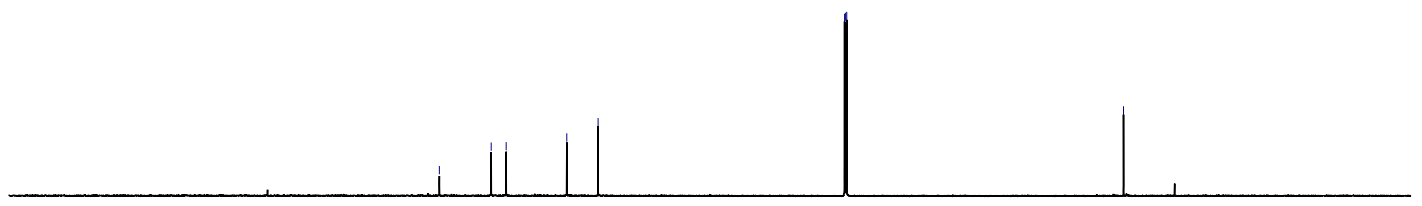

$\begin{array}{lllllllllllllllllllllll}210 & 200 & 190 & 180 & 170 & 160 & 150 & 140 & 130 & 120 & 110 & \begin{array}{c}100 \\ \mathrm{ppm}\end{array} & 90 & 80 & 70 & 60 & 50 & 40 & 30 & 20 & 10 & 0 & -10\end{array}$

Figure S58. ${ }^{13} \mathrm{C}$ NMR of N-methylpyridin-3-amine 


\section{$\stackrel{\infty}{\infty}$}

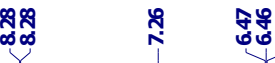

$\stackrel{\mathscr{B}}{m^{\prime}}$

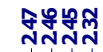

(N)

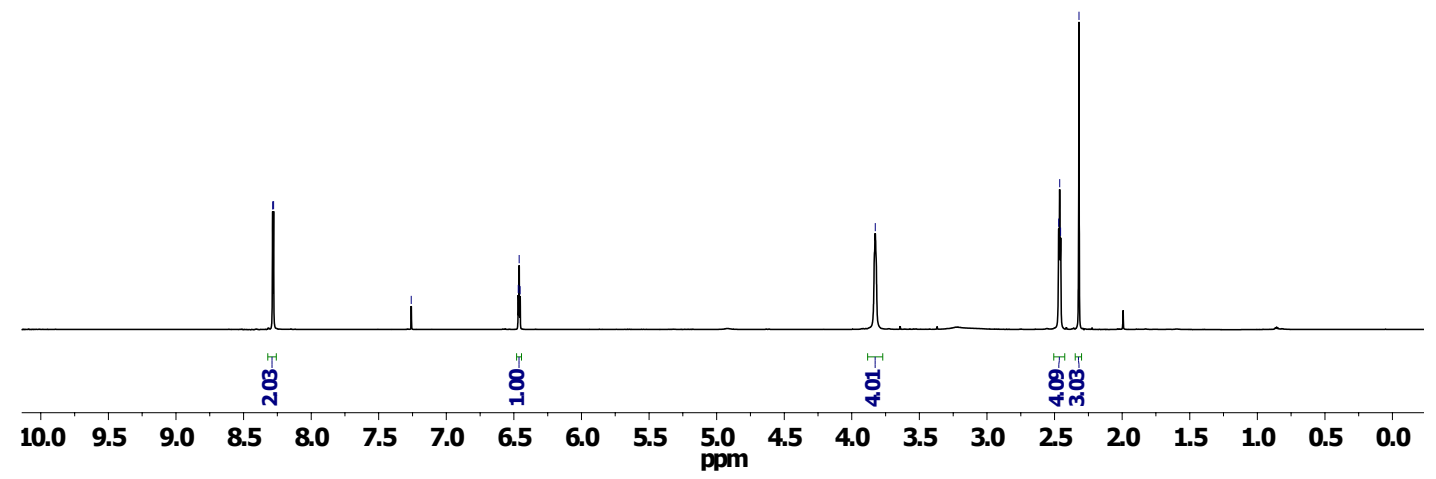

Figure S59. ${ }^{1} \mathrm{H}$ NMR of 2-(4-methylpiperazin-1-yl)pyrimidine
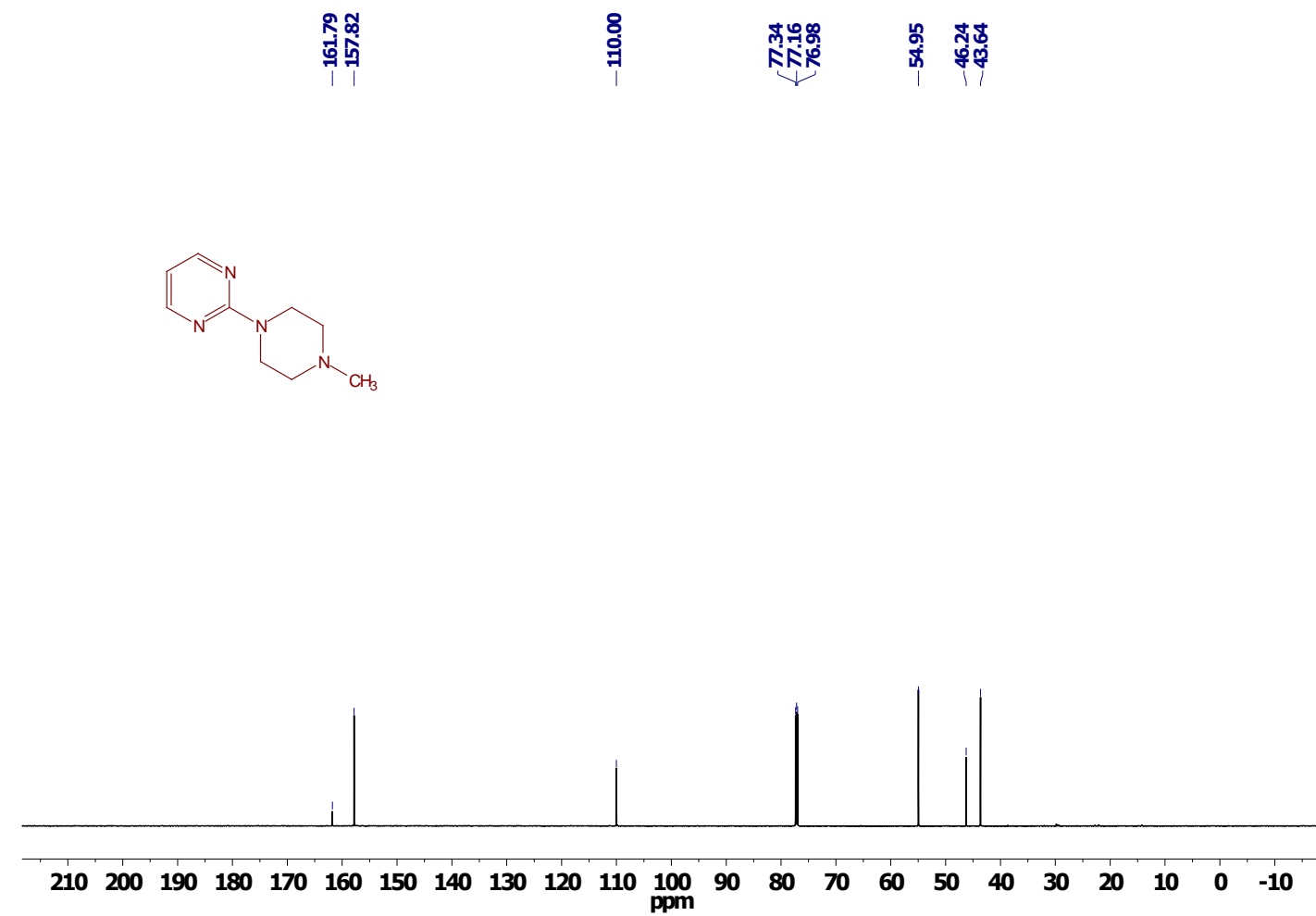

Figure S60. ${ }^{13} \mathrm{C}$ NMR of 2-(4-methylpiperazin-1-yl)pyrimidine 

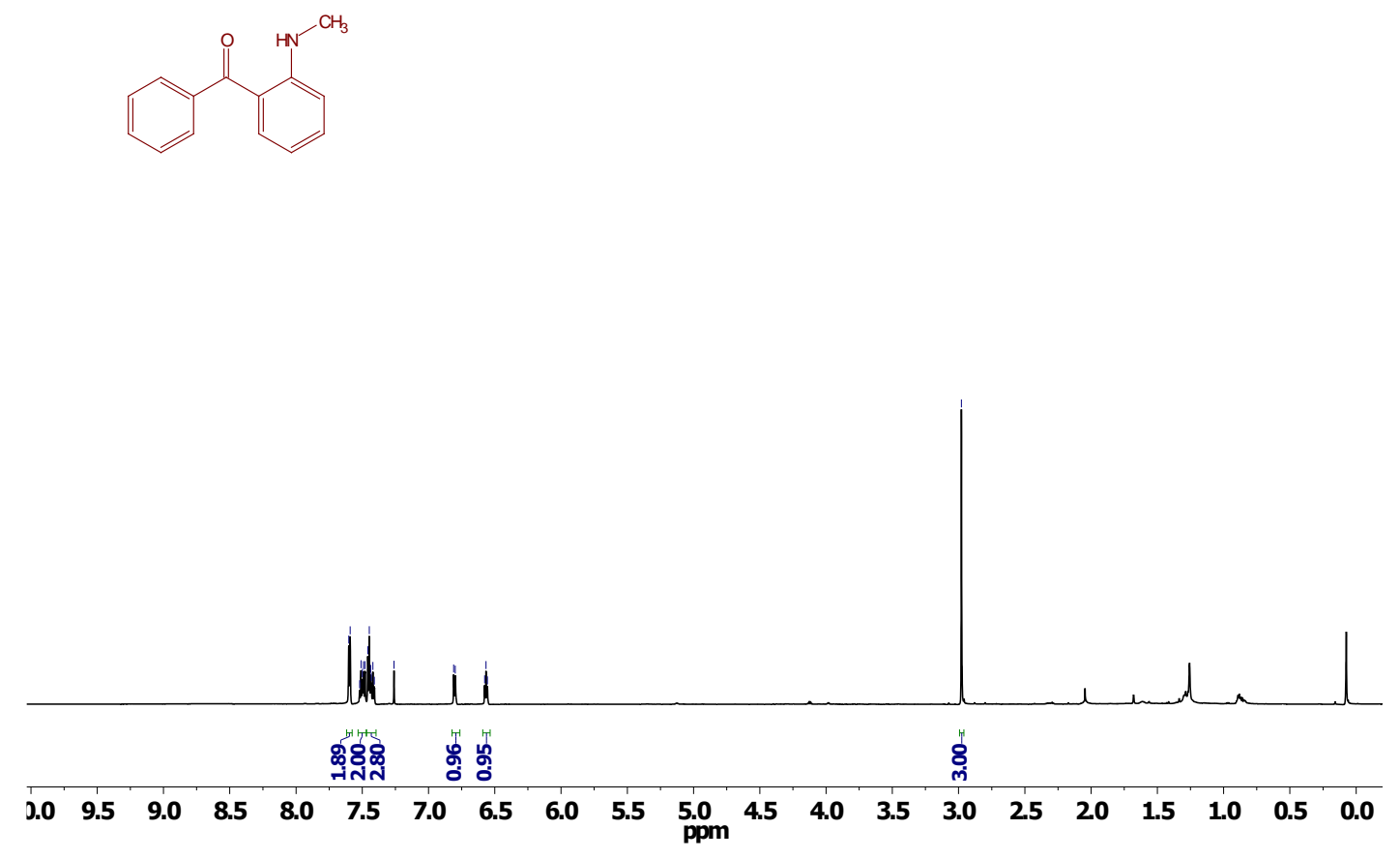

Figure S61. ${ }^{1}$ H NMR 2-(methylamino)phenyl)(phenyl)methanone

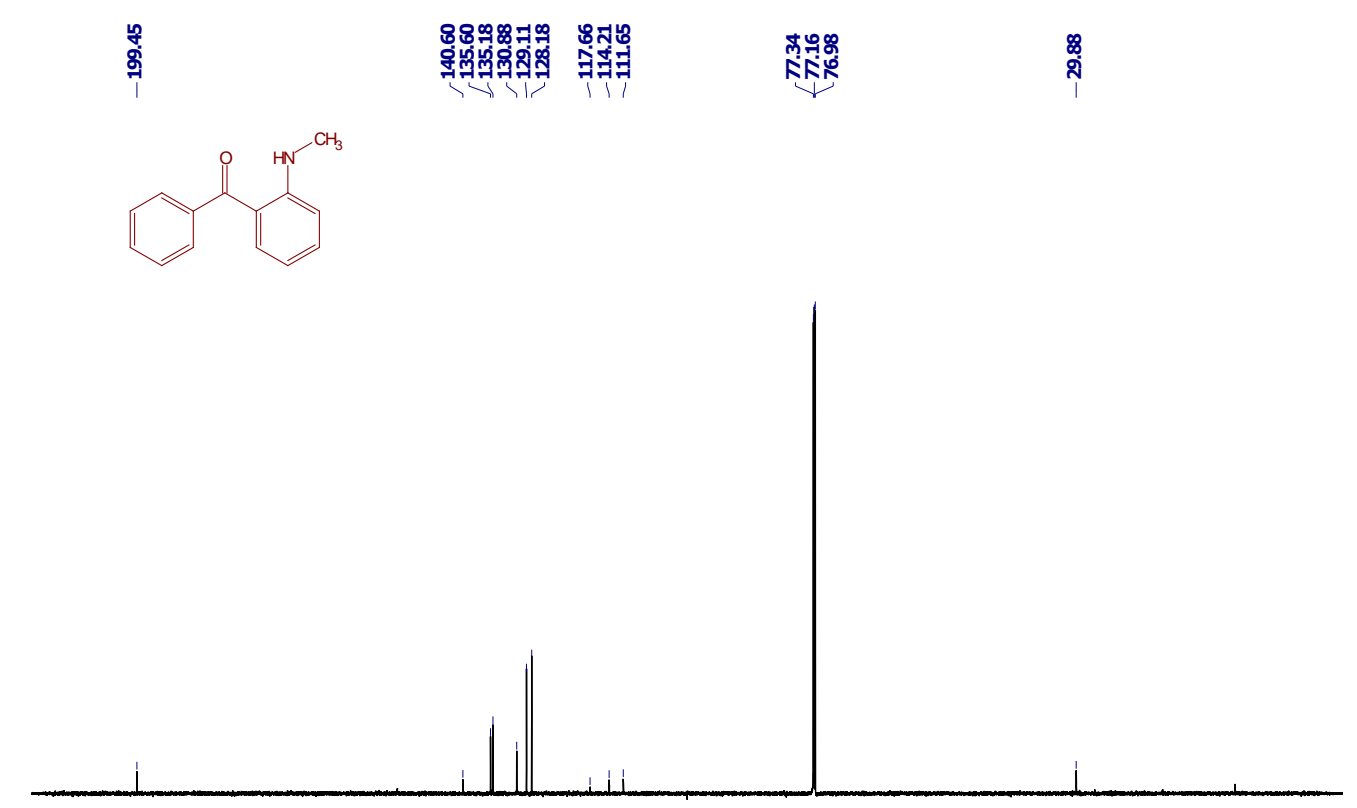

$\begin{array}{lllllllllllllllllllllllll}210 & 200 & 190 & 180 & 170 & 160 & 150 & 140 & 130 & 120 & 110 & 100 & 90 & 80 & 70 & 60 & 50 & 40 & 30 & 20 & 10 & 0 & -10\end{array}$

Figure S62. ${ }^{13} \mathrm{C}$ NMR of 2-(methylamino)phenyl)(phenyl)methanone 


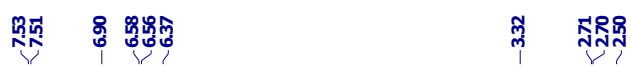
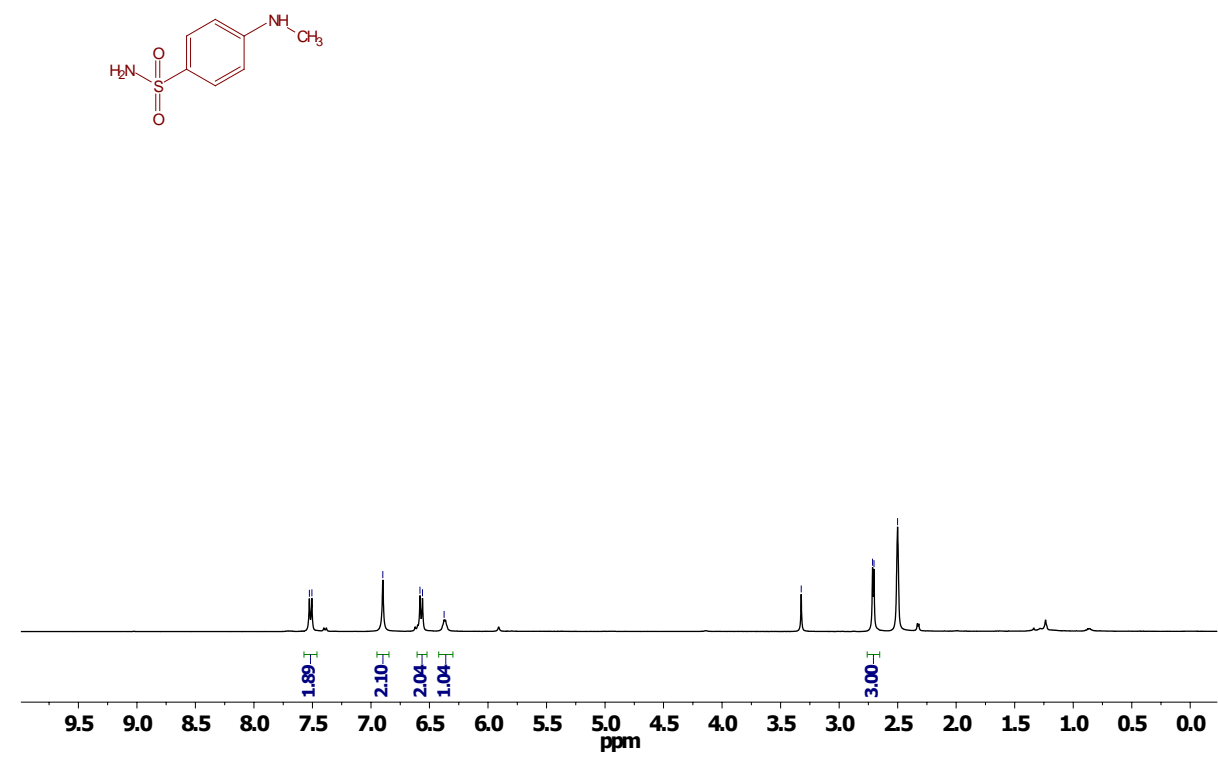

Figure S63. ${ }^{1} \mathrm{H}$ NMR 4-(methylamino)benzenesulfonamide

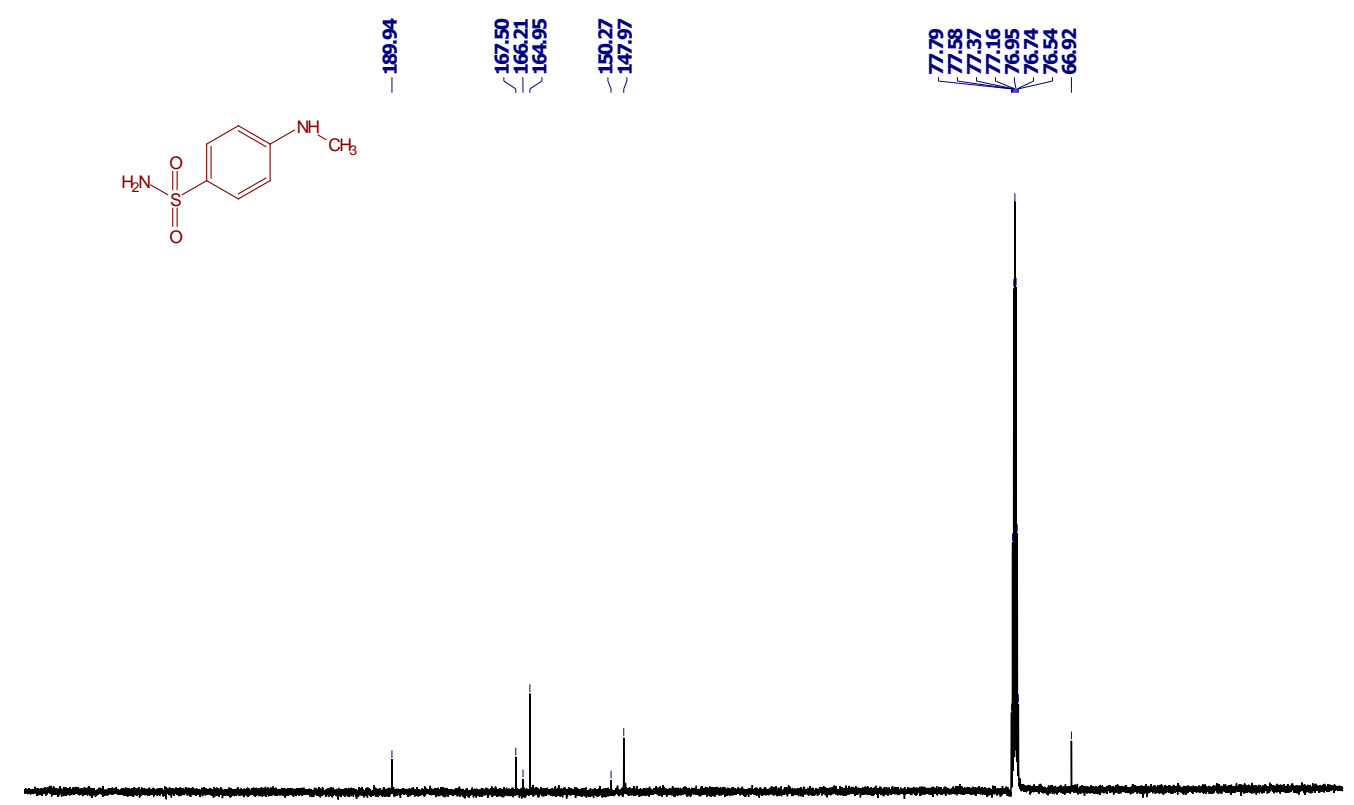

$\begin{array}{llllllllllllllllllllllllllllllll}250 & 240 & 230 & 220 & 210 & 200 & 190 & 180 & 170 & 160 & 150 & \begin{array}{c}140 \\ \mathrm{ppm}\end{array} & 130 & 120 & 110 & 100 & 90 & 80 & 70 & 60 & 50 & 40 & 30 & 20\end{array}$

Figure S64. ${ }^{13} \mathrm{C}$ NMR of 4-(methylamino)benzenesulfonamide 

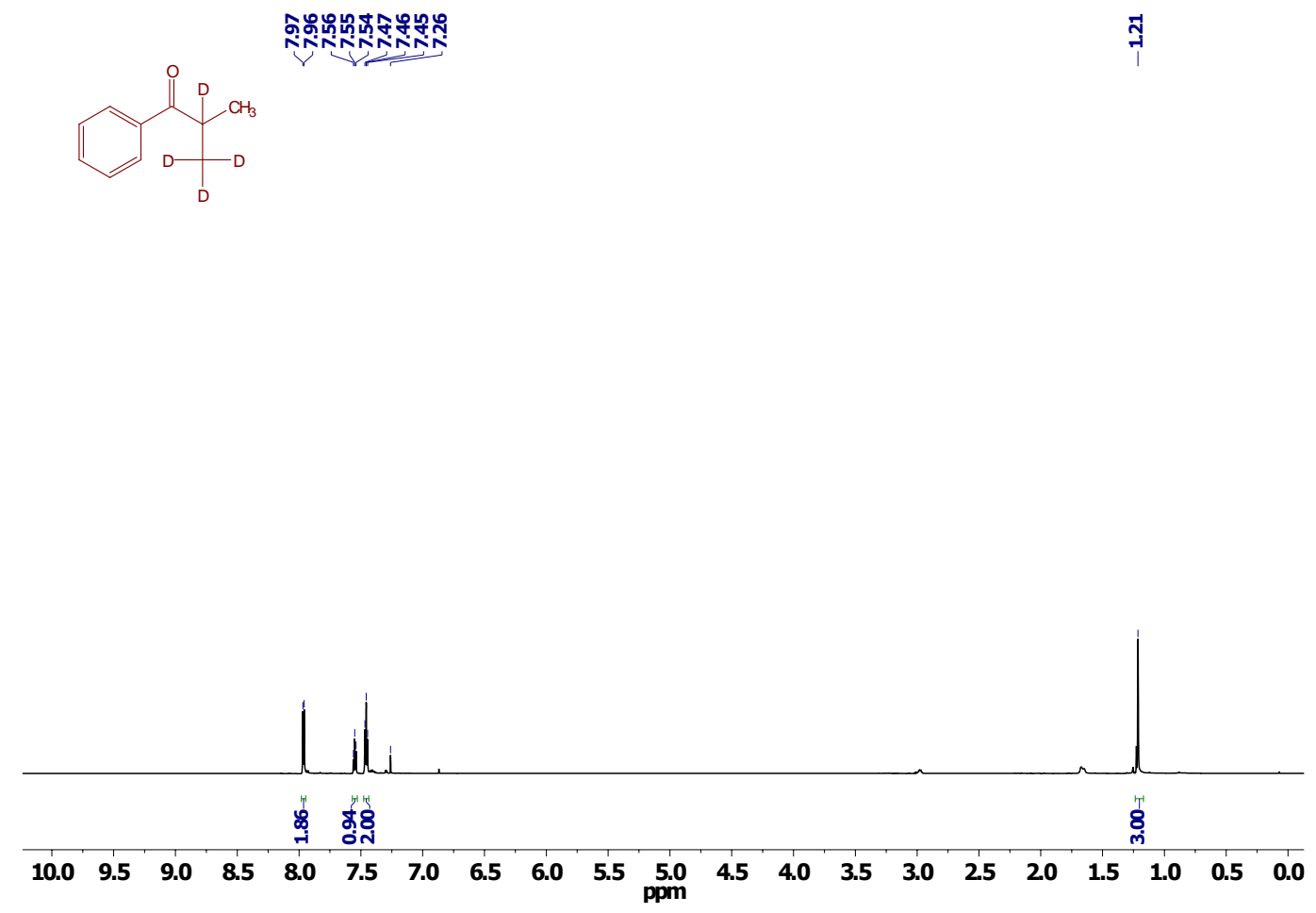

Figure S65. ${ }^{1} \mathrm{H}$ NMR of 1-(4-methoxyphenyl)-2-methylpropan-1-one-2,3,3,3-d4
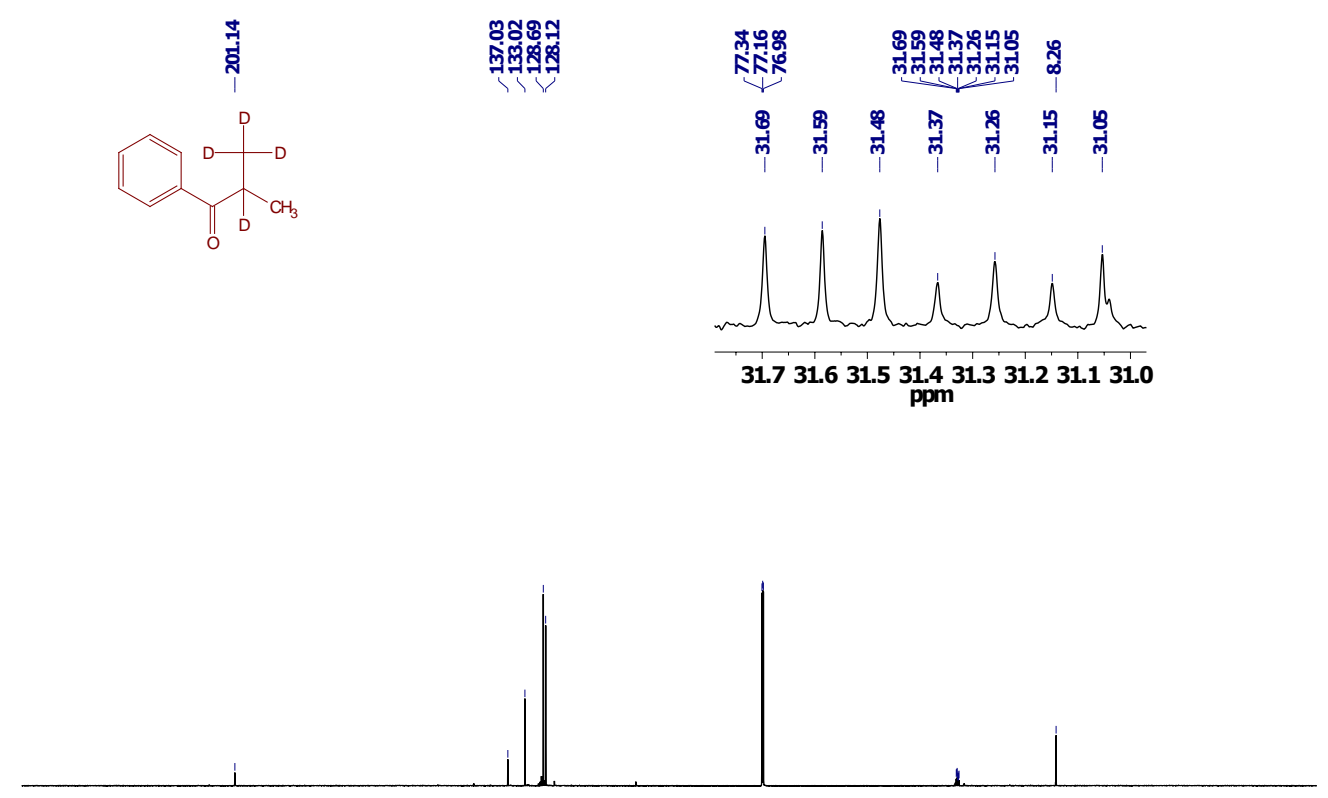

$\begin{array}{llllllllllllllllllllll}50 & 230 & 210 & 190 & 170 & 150 & 130 & 110 & 90 & 70 & 50 & 30 & 10 & -10 & -30 & -5 C\end{array}$

Figure S66. ${ }^{13} \mathrm{C}$ NMR of 1-(4-methoxyphenyl)-2-methylpropan-1-one-2,3,3,3-d4 


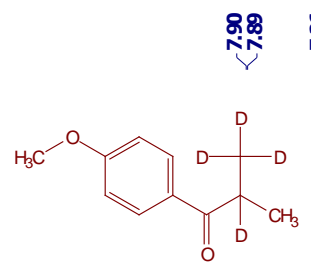

荡

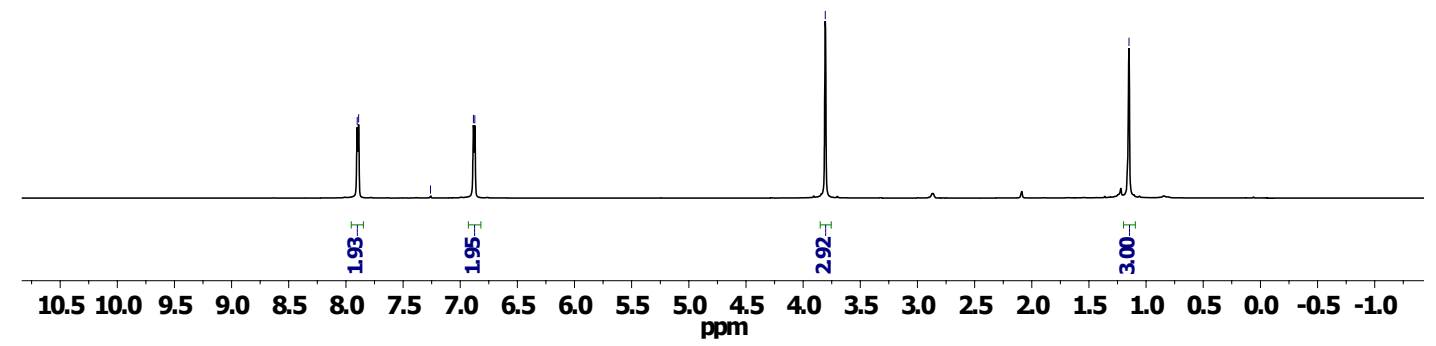

Figure S67. ${ }^{1} \mathrm{H}$ NMR of 1-(4-methoxyphenyl)-2-methylpropan-1-one-2,3,3,3-d4

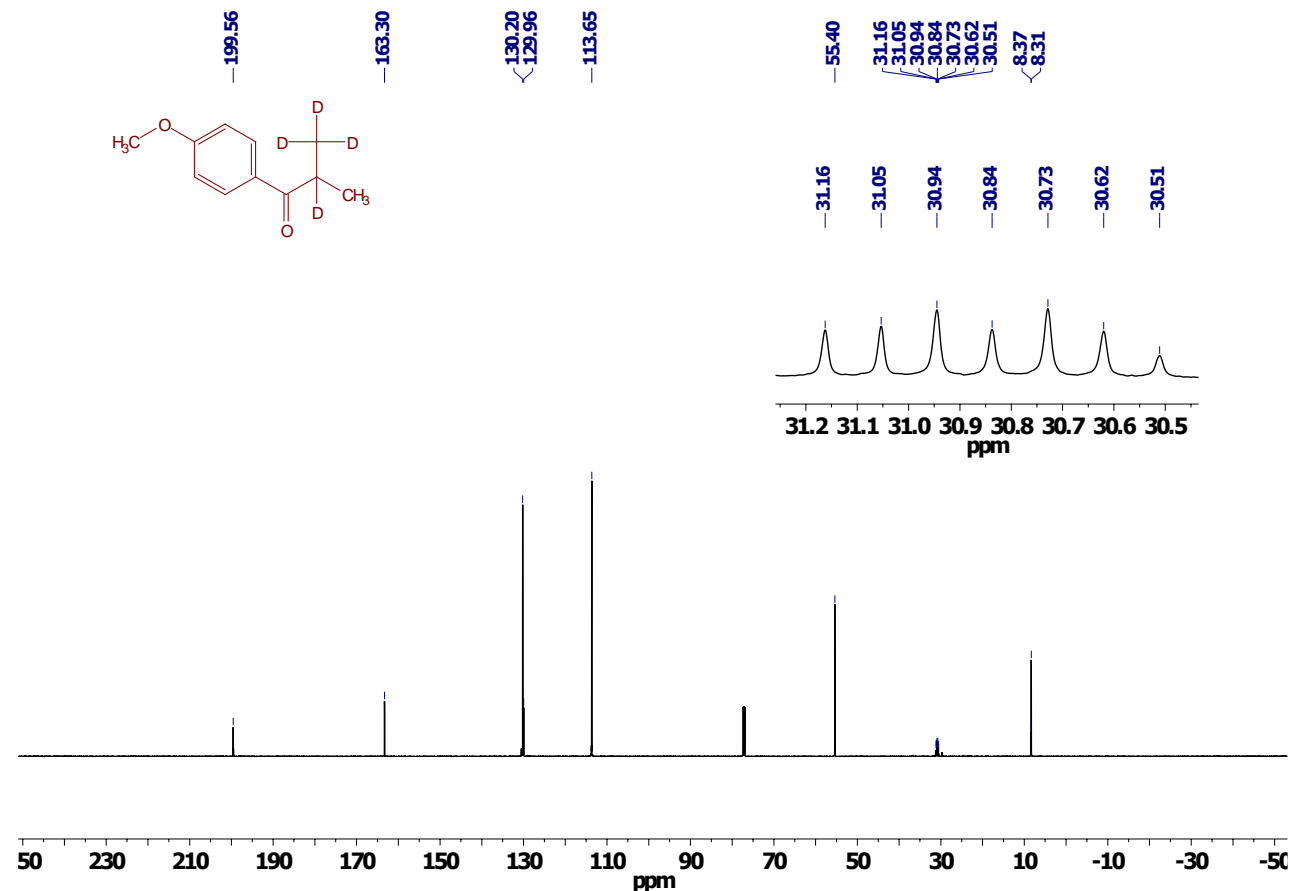

Figure S68. ${ }^{13} \mathrm{C}$ NMR of 1-(4-methoxyphenyl)-2-methylpropan-1-one-2,3,3,3-d4 

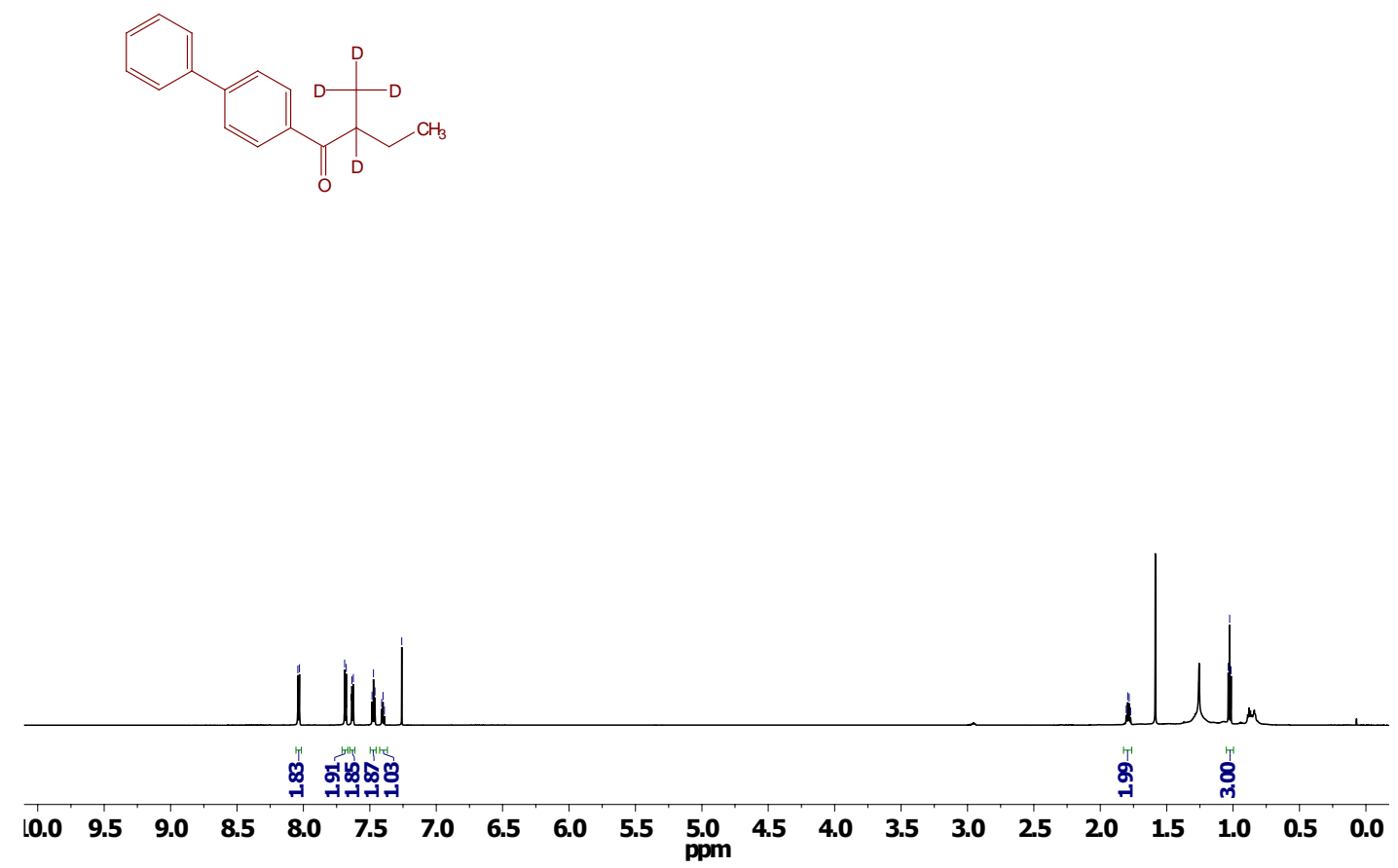

Figure S69. ${ }^{1} \mathrm{H}$ NMR of 1-([1,1'-biphenyl]-4-yl)-2-(methyl-d3)butan-1-one-2-d

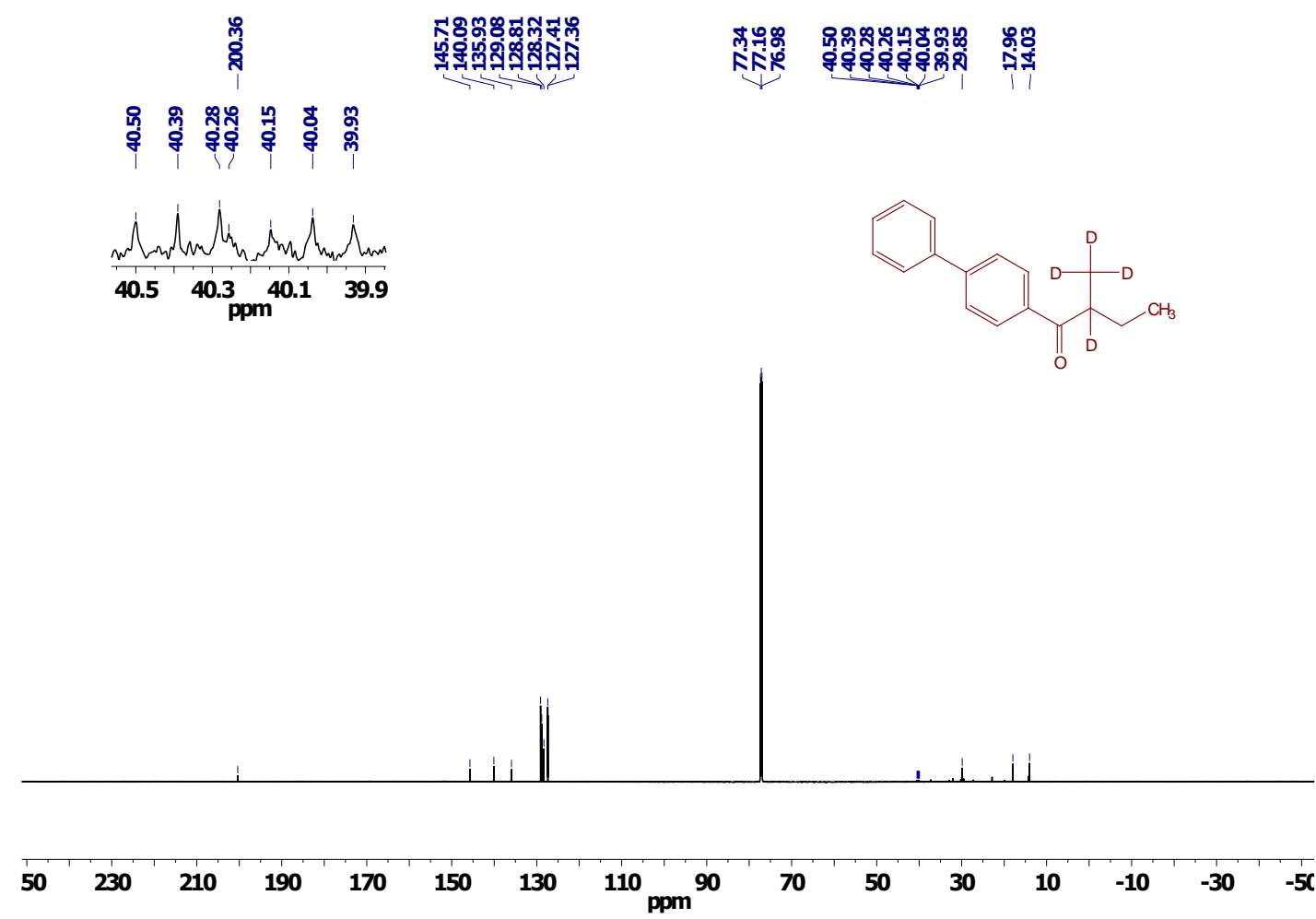

Figure S70. ${ }^{13} \mathrm{C}$ NMR of 1-([1,1'-biphenyl]-4-yl)-2-(methyl-d3)butan-1-one-2-d 